\title{
Control of the leishmaniases
}

Report of a meeting of the WHO Expert Committee on the Control of Leishmaniases, Geneva, 22-26 March 2010 


\section{CONTROL OF THE LEISHMANIASES}

Report of a meeting of the WHO Expert Committee on the Control of Leishmaniases, Geneva, 22-26 March 2010

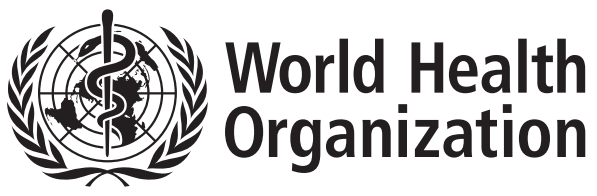


WHO Library Cataloguing-in-Publication Data:

Control of the leishmaniasis: report of a meeting of the WHO Expert Committee on the Control of Leishmaniases, Geneva, 22-26 March 2010.

(WHO technical report series ; no. 949)

1.Leishmaniasis - prevention and control. 2.Leishmaniasis - parasitology. 3.Leishmaniasis - pathology. 4.Leishmaniasis - diagnosis. 5.Leishmaniasis, Cutaneous. I.WHO Expert Committee on the Control of the Leishmaniases. II.World Health Organization. III.Series.

ISBN $9789241209496 \quad$ (NLM classification: WR 350)

ISSN 0512-3054

\section{World Health Organization 2010}

All rights reserved. Publications of the World Health Organization can be obtained from WHO Press, World Health Organization, 20 Avenue Appia, 1211 Geneva 27, Switzerland (tel.: +41 22791 3264; fax: +41 22791 4857; e-mail: bookorders@ who.int). Requests for permission to reproduce or translate WHO publications - whether for sale or for noncommercial distribution - should be addressed to WHO Press, at the above address (fax: +41 22791 4806; e-mail: permissions@ @ who.int).

The designations employed and the presentation of the material in this publication do not imply the expression of any opinion whatsoever on the part of the World Health Organization concerning the legal status of any country, territory, city or area or of its authorities, or concerning the delimitation of its frontiers or boundaries. Dotted lines on maps represent approximate border lines for which there may not yet be full agreement.

The mention of specific companies or of certain manufacturers' products does not imply that they are endorsed or recommended by the World Health Organization in preference to others of a similar nature that are not mentioned. Errors and omissions excepted, the names of proprietary products are distinguished by initial capital letters.

All reasonable precautions have been taken by the World Health Organization to verify the information contained in this publication. However, the published material is being distributed without warranty of any kind, either expressed or implied. The responsibility for the interpretation and use of the material lies with the reader. In no event shall the World Health Organization be liable for damages arising from its use.

This publication contains the collective views of an international group of experts and does not necessarily represent the decisions or the policies of the World Health Organization.

Printed in Switzerland published 2010 


\section{Table of contents}

Members of the Expert Committee, temporary advisers, secretariat vii

Acronyms and abbreviations $\quad x i$

Introduction xii

1. History 1

2. Leishmaniases in humans $\quad 5$

2.1 Clinical forms $\quad 5$

2.1.1 Old World visceral leishmaniasis 5

2.1.2 Old World cutaneous leishmaniasis 7

2.1.3 Old World mucosal leishmaniasis 9

2.1.4 Old World diffuse cutaneous leishmaniasis 9

2.1.5 New World visceral leishmaniasis 9

2.1.6 New World cutaneous leishmaniasis $\quad 10$

2.1.7 New World mucocutaneous leishmaniasis $\quad 10$

2.1.8 New World diffuse cutaneous leishmaniasis $\quad 11$

2.1.9 Disseminated cutaneous leishmaniasis $\quad 11$

2.1.10 Post-kala-azar dermal leishmaniasis $\quad 11$

2.1.11 Leishmania and HIV coinfection 12

$\begin{array}{ll}2.2 \text { Pathology } & 12\end{array}$

2.2.1 General pathology 12

2.2.2 Visceral leishmaniasis 13

2.2.3 Post-kala-azar dermal leishmaniasis 14

2.2.4 Uncomplicated cutaneous leishmaniasis 14

2.2.5 Disseminated cutaneous leishmaniasis 15

2.2.6 Leishmaniasis recidivans 16

2.2.7 Diffuse cutaneous leishmaniasis 16

2.2.8 Mucocutaneous leishmaniasis 16

$\begin{array}{lll}2.3 & \text { Parasitology } & 17\end{array}$

2.3.1 Identification criteria 18

2.3.2 Reference strains 18

2.3.3 Identification methods 20

2.3.4 Taxonomy 21

2.4 Reservoir hosts 22

2.4.1 Definition 22

2.4.2 General aspects of reservoir capacity 22

2.4.3 Incrimination of reservoir hosts 23

2.4.4 Humans as reservoir hosts 24

2.4.5 Domestic and peridomestic reservoir hosts 24

2.4.6 Wild reservoir hosts of the Old World 25

2.4.7 Wild reservoir hosts of the New World 26

2.5 Vectors $\quad 27$

2.5.1 Taxonomy 27

2.5.2 Identification criteria 28

$\begin{array}{lll}2.5 .3 \text { Biology } & 29\end{array}$ 
2.5.4 Incrimination of vectors 33

2.5.5 Vector competence 35

2.6 Epidemiological aspects 36

2.6.1 Major foci and human behaviour $\quad 37$

2.6.2 Socioeconomic factors $\quad 40$

2.6.3 Malnutrition $\quad 40$

2.6.4 Population movement 40

2.6.5 Environmental changes $\quad 41$

2.6.6 Climate change 42

2.6.7 Periodic fluctuations in incidence of disease 43

2.6.8 Epidemiological research and mathematical models 44

2.6.9 Geographical information systems $\quad 45$

2.6.10 Epidemiological surveys of visceral leishmaniasis $\quad 46$

3. Control 49

$\begin{array}{lll}3.1 & \text { Diagnosis } & 49\end{array}$

3.1.1 Visceral leishmaniasis $\quad 49$

3.1.2 Cutaneous leishmaniasis 52

3.1.3 Mucocutaneous leishmaniasis 53

3.1.4 Post-kala-azar dermal leishmaniasis 53

3.1.5 Coinfection with Leishmania and HIV 53

3.2 Treatment and vaccines 54

3.2.1 General considerations 54

3.2.2 Antileishmanial medicines $\quad 55$

$\begin{array}{ll}3.2 .3 & \text { Treatment options } \\ 3.2 .4 & 57\end{array}$

3.2.4 Special situations 65

3.2.5 Prophylactic leishmaniasis vaccines 71

3.2.6 Immunochemotherapy and therapeutic vaccines 73

3.3 Detection 73

3.3.1 Passive case detection 73

$\begin{array}{ll}\text { 3.3.2 Active case detection } & 74\end{array}$

$\begin{array}{lll}3.4 & \text { Control of reservoir hosts } & 75\end{array}$

$\begin{array}{ll}\text { 3.4.1 Humans as reservoir hosts } & 75\end{array}$

3.4.2 Canine reservoir hosts 76

3.4.3 Wild animal reservoir hosts of Old World cutaneous
leishmaniasis

3.4.4 Wild animal reservoir hosts of New World cutaneous
leishmaniasis

$\begin{array}{ll}3.5 \text { Vector control } & 79\end{array}$

$\begin{array}{ll}\text { 3.5.1 General considerations } & 79\end{array}$

3.5.2 Methods 80

3.5.3 Entomological monitoring and evaluation of vector
control operations

3.6 Epidemic response 83

3.6.1 Rapid assessment $\quad 84$

3.6.2 Epidemic preparedness 85

3.6.3 Outbreak response 85

3.7 Socioeconomic aspects of leishmaniasis control 86

3.7.1 Social determinants of risk 87

3.7.2 Cost-effectiveness of control measures 88 
4. Burden of leishmaniases $\quad 91$

4.1 Geographical distribution by country 91

4.2 Estimated burden 104

5. Control strategies by nosogeographical entity 107

5.1 Visceral leishmaniasis caused by $L$. donovani and $L$. infantum

(L. chagasi)

5.1.1 Visceral leishmaniasis caused by L. donovani on the Indian subcontinent

5.1.2 Visceral leishmaniasis in East Africa and the southwest Arabian peninsula caused by $L$. donovani and $L$. infantum

5.1.3 Visceral leishmaniasis caused by L. donovani in other places

5.1.4 Foci of visceral leishmaniasis caused by L. infantum with known or assumed canine reservoir hosts

5.2 Anthroponotic cutaneous leishmaniasis caused by L. tropica

5.3 Sporadic cutaneous leishmaniasis caused by L. tropica and related species

5.4 Zoonotic cutaneous leishmaniasis caused by L. major

5.5 Zoonotic cutaneous leishmaniasis in the East African highlands caused by L. aethiopica

5.6 Cutaneous leishmaniasis caused by L. peruviana 119

5.7 Cutaneous leishmaniasis caused by L. guyanensis 121

5.8 Cutaneous and mucocutaneous leishmaniasis caused by $L$. panamensis

5.9 Cutaneous and mucocutaneous leishmaniasis caused by $L$. braziliensis

5.10 Cutaneous leishmaniasis caused by L. mexicana and related species

5.11 Cutaneous leishmaniasis caused by L. infantum 125

5.12 Cutaneous leishmaniasis caused by other New World species 125

6. Organization of control

6.1 Control of leishmaniasis as part of primary health care 128

6.1.1 Community participation 129

6.1.2 Social mobilization and communication 129

6.2 Definition of national plans

6.2.1 Purpose and implementation of national control programmes

6.2.2 Collection of epidemiological data

6.2.3 Definition of control strategies and activities 133

6.2.4 Intersectoral coordination 133

6.2.5 Formal adoption of the national control strategy or plan 133

6.3 Surveillance

6.4 Pharmacovigilance 
7. International coordination 139

$\begin{array}{lll}7.1 & \text { Reporting } & 139\end{array}$

7.2 Technical partners 139

7.3 Intercountry programmes for advocacy and awareness-raising 140

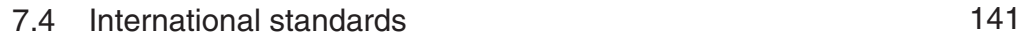

8. Health education and training 143

8.1 Health education 143

8.2 Training 144

9. Research 149

9.1 Field research 149

9.2 Laboratory research 150

9.3 Drug and vaccine research and development 150

9.3.1 What products are needed? 150

9.3.2 Challenges to the development and use of
antileishmanial treatment

9.3.3 Input of other approaches 152

10. Recommendations 153

Annex 1. Labelling of Leishmania isolates, identification centres and sources of standards

Annex 2. Methods for isolation and cryopreservation of Leishmania 165

Annex 3. WHO recommended case definitions 175

Annex 4. Procedures for splenic aspiration and grading of parasites 177

Annex 5. Performance of the rK39 rapid diagnostic test 181

Annex 6. Costs of medicines in current use for the treatment of leishmaniases 


\section{Members of the Expert Committee, temporary advisers, secretariat}

\section{Members ${ }^{1}$}

Professor Richard W. Ashford, former Professor of Parasite and Vector Biology at Liverpool School of Tropical Medicine, United Kingdom

Dr Caryn Bern (Rapporteur), Division of Parasitic Diseases and Malaria, Center for Global Health, Centers for Disease Control and Prevention, Atlanta, Georgia, USA

Professor Marleen Boelaert, Department of Public Health, Institute of Tropical Medicine, Antwerpen, Belgium

Emeritus Professor Anthony Bryceson (Chairman), Department of Infectious and Tropical Diseases, London School of Hygiene and Tropical Medicine, United Kingdom

Dr François Chappuis, Division of International and Humanitarian Medicine, Geneva University Hospitals, Switzerland

Professor Simon Croft, Department of Infectious and Tropical Diseases, London School of Hygiene and Tropical Medicine, United Kingdom

Professor Jean-Pierre Dedet, Université Montpellier 1 and National Reference Centre for Leishmania, Montpellier, France

Dr Philippe Desjeux, institute for One World Health, San Francisco, CA, USA

\footnotetext{
1 Unable to attend: Professor Yahya Dowlati, Centre for Research and Training in Skin Diseases and Leprosy, Tehran University of Medical Sciences, Islamic Republic of Iran; Dr Elizabeth F. Rangel, Instituto Oswaldo Cruz/ Fundação Oswaldo Cruz, Rio de Janeiro, Brazil
} 
Dr Luigi Gradoni, Department of Infectious, Parasitic and Immunomediated Diseases, Istituto Superiore di Sanità, Rome, Italy

Professor Robert R. Killick-Kendrick, former Professor at the Department of Biology, Imperial College at Silwood Park, Ascot, Berkshire, United Kingdom

Professor Elmer Alejandro Llanos-Cuentas, Alexander von Humbodt Institute of Tropical Medicine, Peruana Cayetano Heredia University, Lima, Peru

Dr Rogelio López-Vélez, Tropical Medicine and Clinical Parasitology Unit, Infectious Diseases Department, Hospital Ramón y Cajal, Madrid, Spain

Professor Farrokh Modabber, Medicines For Neglected Diseases, Geneva, Switzerland

Professor Suman Rijal, B. P. Koirala Institute of Health Sciences, Dharan, Sunsari, Nepal

Professor Afif Ben Salah, Epidemiology Laboratory, Pasteur Institute Tunis, Tunis-Belvedere, Tunisia

Dr Poonam Salotra, Institute of Pathology, Indian Council of Medical Research, Safdarjung Hospital Campus, New Delhi, India

Professor Nancy Gore Saravia (Vice-chairperson), Scientific Director, WHO Collaborating Centre for Leishmaniasis, Scientific Director CIDEIM, Cali, Colombia

Professor Jeffrey Jon Shaw, Parasitology Department, University of São Paulo, Brazil

Professor Shyam Sundar (Co-rapporteur), Institute of Medical Sciences, Banaras Hindu University, Varanasi, India

Emeritus Professor Chandreshwar P. Thakur, Patna Medical College, India

Dr Dinesh Mondal, International Centre for Diarrhoeal Disease Research, Dhaka, Bangladesh

Professor Guilherme L. Werneck, Department of Epidemiology, Federal University of Rio de Janeiro, Brazil 


\section{Advisers}

Professor Hannah Akuffo, Karolinska Institute and Swedish International Development Agency, Stockholm, Sweden

Dr Abraham Aseffa, Deputy Director, Armauer Hansen Research Institute, Addis Ababa, Ethiopia

Dr Pierre Buffet, Parasitology Service, Pitié-Salpêtrière Hospital and UMR945 INSERM, Paris 6 University, Paris, France

Dr Dia-Eldin Elnaiem, University of Maryland Eastern Shore, Princess Anne, Maryland, USA

Professor Nirmal K. Ganguly, Indian Council of Medical Research, New Delhi, India

Dr Ahmed Mudawi Musa Mohammed, Institute of Endemic Diseases, University of Khartoum, Sudan

Dr Koert Ritmeijer, University of Amsterdam, Netherlands

\section{Heads of WHO collaborating centres}

Dr Carmen Cañavate, WHO Collaborating Centre for Leishmaniasis, Carlos III Health Institute, Majadahonda, Madrid, Spain

Professor Hechmi Louzir, WHO Collaborating Centre for Leishmaniasis, Pasteur Institute, Tunis, Tunisia

\section{Representatives of WHO regional offices}

Dr R. Andraghetti, Medical Officer, Communicable Diseases Unit, WHO Regional Office for Europe, Copenhagen, Denmark

Dr D. Argaw Dagne, Medical Officer, WHO Country Office, Addis Ababa, Ethiopia

Dr R. Ben Ismail, Medical Officer, Communicable Diseases, WHO Regional Office for the Eastern Mediterranean, Cairo, Egypt

Dr S. Bhattacharya, Communicable Diseases, WHO Regional Office for South-East Asia, New Delhi, India

Dr R. Gusmao, Medical Officer, Communicable Diseases, WHO Pan American Health Organisation, Rio de Janeiro, Brazil 


\section{WHO Secretariat}

Dr J. Alvar, Medical Officer, Leishmaniasis Programme, Innovative and Intensified Disease Management, Department of $\mathrm{Ne}-$ glected Tropical Diseases

Dr B. Arana, Medical Officer, Special Programme for Research and Training in Tropical Diseases

Dr M. den Boer, Leishmaniasis Programme, Innovative and Intensified Disease Management, Department of Neglected Tropical Diseases (Temporary adviser)

Dr J. Jannin, Coordinator, Innovative and Intensified Disease Management, Department of Neglected Tropical Diseases

Professor G. Matlashewski, Leader, Special Programme for Research and Training in Tropical Diseases

Dr P. Olliaro, Leader, Special Programme for Research and Training in Tropical Diseases

Dr R. Velayudhan, Vector Ecology and Management, Department of Control of Neglected Tropical Diseases

Dr L. Savioli, Director, Department of Neglected Tropical Diseases

Professor I.D. Vélez, Leishmaniasis Programme, Innovative and Intensified Disease Management, Department of Neglected Tropical Diseases (Short-term consultant) 


\section{Acronyms and abbreviations}

BCG

DDT

ELISA

HIV

IFAT

IL

NNN

PCR

PKDL

TNF

USA

USAMRU

WHO

WHOPES bacillus Calmette-Guérin

dichlorodiphenyltrichloroethane

enzyme-linked immunosorbent assay

human immunodeficiency virus

immunofluorescence antibody test

interleukin

Novy-MacNeal-Nicolle

polymerase chain reaction

post-kala-azar dermal leishmaniasis

tumour necrosing factor

United States of America

United States Army Medical Research Unit

World Health Organization

WHO Pesticide Evaluation Scheme 


\section{Introduction}

Leishmaniasis is still one of the world's most neglected diseases, affecting largely the poorest of the poor, mainly in developing countries; 350 million people are considered at risk of contracting leishmaniasis, and some 2 million new cases occur yearly. In the past 10 years, major scientific breakthroughs have been made in the treatment, diagnosis and prevention of leishmaniasis, and the prices of several key medicines have been reduced. These developments have facilitated implementation of sustainable national and regional control programmes; however, functioning control programmes are still rare, and mortality and morbidity from leishmaniasis worldwide show a worrying increasing trend.

A strategic milestone was achieved in 2007, when the World Health Assembly approved Resolution 60.13 on the control of leishmaniasis. This resolution calls for the creation of conditions to enable WHO to take a leading role in providing technical assistance for the initiation, maintenance and expansion of leishmaniasis control programmes. One of the recommendations was to draft guidelines on the prevention and management of leishmaniasis and to update the WHO Technical Report on the control of leishmaniasis prepared by the WHO Expert Committee in 1990. For this purpose, the Expert Committee reconvened in Geneva on 22-26 March 2010 to review the 1990 guidelines. The new edition contained here reflects the latest scientific and other relevant developments in the field of leishmaniasis.

This revised and updated edition includes new therapeutic recommendations for visceral and cutaneous leishmaniasis, recommendations on the use of rapid diagnostic tests, details on the management of Leishmania-HIV coinfection and consideration of social factors and climate change as risk factors for increased spread. Recommendations for research include the furtherance of epidemiological knowledge of the disease and clinical studies to address the lack of an evidence-based therapeutic regimen for cutaneous and mucocutaneous leishmaniases and post-kala-azar dermal leishmaniasis (PKDL).

The experts' most important conclusion is that adequate control of leishmaniasis worldwide is feasible with the medicines and diagnostic tools currently available. In line with the Resolution, however, it was recognized that there 
is a crucial lack of funding, political commitment and national and international cooperation. WHO is strongly encouraged to take the lead in establishing effective control programmes in affected areas, where they are most urgently needed. This report not only provides clear guidance on implementation but should also raise awareness about the global burden of leishmaniasis and its neglect. 



\section{History}

At the turn of the nineteenth century, Cunningham, Borovsky, Leishman, Donovan, Wright, Lindenberg and Vianna each independently identified the parasite that causes leishmaniasis, to which Ronald Ross gave the generic name Leishmania. In 1904, Cathoire and Laveran found Leishmania in children with infantile splenic anaemia. Nicolle named the parasite $L$. infantum, identified its reservoir in dogs in Tunis in 1908 and cultured it in the laboratory. Carini identified Leishmania in mucosal lesions of patients with leishmaniasis in Brazil in 1912. In 1914, the Russians Yakimoff and Shakor distinguished the parasites that caused the dry, urban and wet, rural forms of cutaneous leishmaniasis in Central Asia. Bramachari described PKDL in India in 1922. In the early 1940s, Swaminath, Shortt and Anderson in India and Adler and Ber in Palestine demonstrated the transmission of $L$. donovani and 'L. tropica' (probably L. major) by phlebotomine sandflies. Gradually, the clinical and geographical features of the human disease were supplemented by studies of animal reservoirs and vectors, the behaviour of Leishmania in experimental animals and the ecology of natural cycles of leishmaniasis, strengthening the basis for classification and for understanding transmission to humans. Genetic speciation had to wait for the introduction of iso-enzyme analysis in the 1970s and DNA hybridization in the early 1980s.

The original techniques for demonstrating amastigotes in smears of splenic aspirates and skin lesions for diagnosis are still reference methods. In the $1990 \mathrm{~s}$, the detection of kinetoplast DNA by polymerase chain reaction (PCR) greatly increased sensitivity and permitted species diagnosis in tissue specimens and blood. Early immunodiagnostic tests and the aldehyde test lacked sensitivity and specificity and were replaced by indirect immunofluorescence and enzyme-linked immunosorbent assays (ELISAs) in the 1970s; these in turn have been replaced in the field by two techniques that do not require a laboratory: the direct agglutination of promastigotes in the 1980s and immunochromatographic detection by dip-stick of a cloned recombinant K39 antigen in the mid-1990s.

Trivalent antimonials were introduced for the treatment of cutaneous and mucocutaneous leishmaniases by Vianna in Brazil in 1912 and for visceral 
leishmaniasis by Di Cristina and Caronia in Italy in 1915. In 1922, Bramachari introduced urea stibamine, the first of a number of much safer pentavalent antimonials, which have remained the mainstay of treatment of all forms of leishmaniasis. It was known that the curative dose was different in different countries, but the lack of clinical trials and fear of toxicity led to unsound treatment regimens. In the 1970s in Kenya and the 1980s in Bihar, India, patients with visceral leishmaniasis increasingly failed to respond to treatment, and isolates of Leishmania (populations of parasites belonging to the same (sub) species) proved resistant to antimonials. A need for safer, more effective medicines drove a search for new compounds, leading to the first registration for visceral leishmaniasis of liposomal amphotericin B in 1996, miltefosine in 2004 and paromomycin in 2006.

McCombie Young, in 1920-1923, controlled a severe post-influenza recrudescence of the kala-azar (visceral leishmaniasis) epidemic in Assam by finding cases and transporting the patients to a treatment centre, where over 80000 people received intravenous trivalent antimonials twice weekly for 3 months. This operation was a cost-effective public health success. Spraying of gerbil burrows with insecticide controlled zoonotic cutaneous leishmaniasis in an endemic area of Turkmenistan in the 1940s but failed in Iran. Largescale control of vectors of leishmaniasis with residual insecticides was introduced in the 1950s, and their use, together with case finding and treatment, achieved control of anthroponotic cutaneous leishmaniasis in the USSR and Central Asia, although some areas of Central Asia have seen a resurgence in recent years. At the same time, leishmaniasis almost disappeared in parts of the Middle East and India as a consequence of antimalarial insecticide spraying. In the $1970 \mathrm{~s}$, to control zoonotic cutaneous leishmaniasis in Uzbekistan, gerbils were eliminated by poisoning, and their habitats were destroyed by repeated physical destruction of burrows and their sandfly populations with heavy caterpillar tractors, followed by flooding. The most successful campaign to control zoonotic visceral leishmaniasis by reservoir control was carried out in eastern China during the 1950s to 1980s, which involved compulsory destruction of dogs, accompanied by house spraying with residual insecticides, case finding and treatment.

Epidemic anthroponotic L. donovani visceral leishmaniasis in the GangesBrahmaputra Basin in India, which almost disappeared during the malaria eradication programme of the 1950s, returned in the 1970s and has persisted. Endemic visceral leishmaniasis led to epidemics in Kenya in the 1950s and 1960s and, in association with civil war, in the Sudan in the 1980s and 1990s, causing thousands of deaths. Both epidemics have spread into Ethiopia. Coinfection with human immunodeficiency virus (HIV) was first reported in European Mediterranean countries in the mid-1980s and extended progressively to other regions. Visceral leishmaniasis presented atypical clinical 
features as an opportunistic infection in adults with HIV, with high rates of relapse and mortality. The prevalence of zoonotic visceral leishmaniasis has been increasing in some South American countries and becoming urbanized. Civil war led to an epidemic that caused hundreds of thousands of cases of cutaneous leishmaniasis caused by L. tropica in Kabul, Afghanistan, in the 1990s, and this was followed by large outbreaks caused by L. major in refugee camps in Pakistan. Leishmania braziliensis, and other species, formerly considered parasites in South American forests, have adapted to deforestation by finding new vectors and reservoir hosts, leading to increased numbers of urban cases of cutaneous and mucocutaneous leishmaniasis in Brazil and other South American countries. 



\section{Leishmaniasis in humans}

\subsection{Clinical forms}

\subsubsection{Old World visceral leishmaniasis}

Visceral leishmaniasis is caused by parasites of the $L$. donovani- $L$. infantum complex (see section 2.3.4, Figure 1 and Table 1). A few cases caused by L. tropica have been reported. Most infections are asymptomatic, although longitudinal follow-up has shown that some victims eventually develop clinical visceral leishmaniasis. Malnutrition and immune suppression, notably HIV infection, predispose to clinical disease. Visceral leishmaniasis may be endemic, sporadic or epidemic, with different clinical features in each situation.

In areas endemic for visceral leishmaniasis, the disease tends to be relatively chronic, and children are especially affected. Until recently, the age group most affected by endemic visceral leishmaniasis caused by L. infantum in southern Europe, North Africa and West and Central Asia was 1-4 years. Since the advent of HIV infection and increased use of immunosuppression for transplantation and chemotherapy, however, about half the cases in Europe are now in adults. In endemic areas of East Africa and India, the highest incidence is in children and young adults. In many countries, more male than females cases are reported (see sections 2.6 and 3.7). The incubation period ranges from 10 days to over 1 year, and the onset of the disease is usually gradual. The common symptoms are fever, malaise, shivering or chills, weight loss, anorexia and discomfort in the left hypochondrium. The common clinical signs are non-tender splenomegaly, with or without hepatomegaly, wasting and pallor of mucous membranes. Lymphadenopathy may be present (especially in the Sudan) and may be the only clinical manifestation. Darkening of the skin of the face, hands, feet and abdomen is typically found in India (the Hindi name, kala-azar, means 'black fever' or 'deadly fever'). In the Sudan and, rarely, in East Africa, a cutaneous nodule or ulcer or a mucosal lesion may be present, containing Leishmania. Signs of malnutrition (oedema, skin and hair changes) develop as the disease progresses. Intercurrent infections are common. 


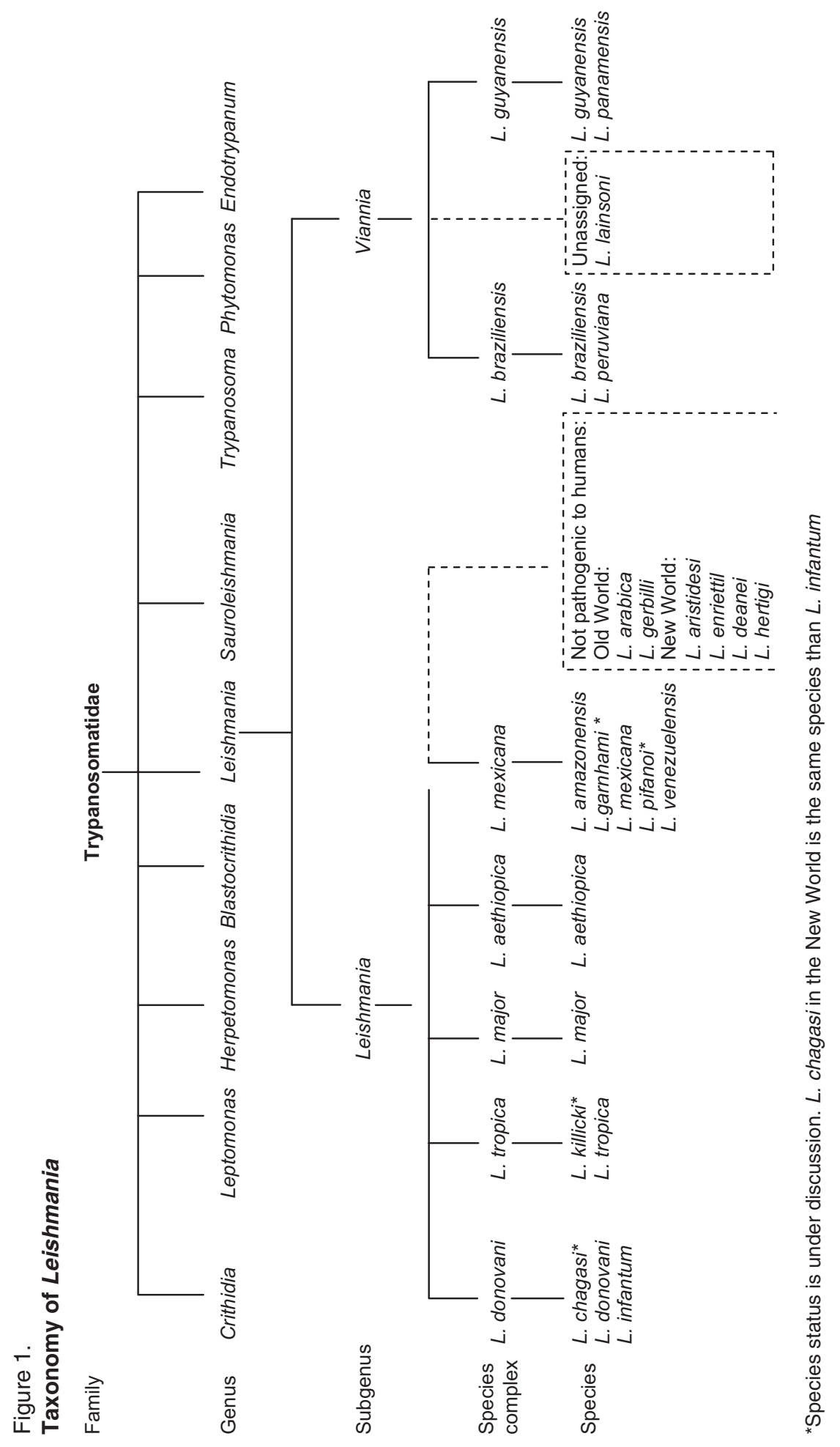


Table 1.

\section{Leishmania found in humans}

\begin{tabular}{|c|c|c|c|c|}
\hline Subgenus & L. (Leishmania) & L. (Leishmania) & L. (Viannia) & L. (Viannia) \\
\hline Old World & $\begin{array}{l}\text { L. donovani } \\
\text { L. infantum }\end{array}$ & $\begin{array}{l}\text { L. major } \\
\text { L. tropica } \\
\text { L. killicki } \\
\text { L. aethiopica } \\
\text { L. infantum }\end{array}$ & & \\
\hline New World & L. infantum & $\begin{array}{l}\text { L. infantum } \\
\text { L. mexicana } \\
\text { L. pifanoia } \\
\text { L. venezuelensis } \\
\text { L. garnhami } \\
\text { L. amazonensis }\end{array}$ & $\begin{array}{l}\text { L. braziliensis } \\
\text { L. guyanensis } \\
\text { L. panamensis } \\
\text { L. shawi } \\
\text { L. naïffi } \\
\text { L. lainsoni } \\
\text { L. lindenbergi } \\
\text { L. peruviana } \\
\text { L. colombiensis }\end{array}$ & $\begin{array}{l}\text { L. braziliensis } \\
\text { L. panamensis }\end{array}$ \\
\hline Principal tropism & Viscerotropic & Dermotropic & Dermotropic & Mucotropic \\
\hline
\end{tabular}

a Species status is under discussion

b Taxonomic position is under discussion

Sporadic visceral leishmaniasis may occur in nonindigenous people of any age who enter an endemic area. Such cases may be acute, with abrupt onset of fever beginning 3 weeks to 2 years after exposure. The disease may progress rapidly, with chills, high undulating fever, often with two peaks per day, drenching sweats, rapid weight loss and profound malaise. These patients are more likely to develop the rare complications of severe acute haemolytic anaemia, acute renal damage and mucosal haemorrhage.

In epidemic anthroponotic (transmissible from human to human) visceral leishmaniasis, people of all ages are susceptible, except those who acquired immunity during a previous epidemic. Acute forms can occur, and mortality is usually high.

Coinfection with HIV has changed the classical picture of visceral and other forms of leishmaniasis. (See sections 2.1.11 and 3.2.4.)

\subsubsection{Old World cutaneous leishmaniasis}

The clinical features of cutaneous leishmaniasis tend to vary between and within regions, reflecting different species of parasite or the type of zoonotic cycle concerned, immunological status and also perhaps genetically determined responses of patients. A 'classical' lesion starts as a papule or nodule 
at the site of inoculation; it grows slowly, taking at least 1 week to reach its final size. A crust develops centrally, which may fall away, exposing an ulcer up to $5 \mathrm{~cm}$ in diameter with a raised edge and variable surrounding induration, which heals gradually over months or years, leaving a depressed scar with altered pigmentation. Satellite nodules at the edge of the lesion are common. Clinicians should be aware of the wide variety of clinical presentations possible.

Cutaneous leishmaniasis of the Old World is caused by five species of Leishmania: L. infantum, L. tropica, L. major, L. aethiopica and L. donovani.

Cutaneous lesions caused by L. infantum are seen throughout its distribution, most notably in the Mediterranean Basin. Leishmania infantum is the most frequent cause of cutaneous leishmaniasis in southern Europe. The lesions are most commonly single nodules, with little inflammation, although typical ulcers also occur. In the absence of immunosuppresion, there are no signs or previous history of visceral leishmaniasis. The lesions heal spontaneously within about 1 year and seem to confer immunity. Leishmania tropica is also present in Greece.

Cutaneous leishmaniasis caused by L. tropica (previously known as anthroponotic or urban anthroponotic cutaneous leishmaniasis) produces painless, frequently multiple, dry ulcers of the skin, which usually heal spontaneously within about 1 year, or sometimes longer, often leading to disfiguring scars. The incubation period is usually $2-8$ months.

Leishmaniasis recidivans, also known as lupoid or tuberculoid leishmaniasis, is a chronic form of anthroponotic cutaneous leishmaniasis that may last for many years. The slowly progressing lesions, usually on exposed areas, are characterized by a scar with peripheral activity. Untreated, the disease is destructive and disfiguring. The scarcity of amastigotes in the lesion can easily lead to delayed or incorrect diagnosis.

Cutaneous leishmaniasis caused by L. major (previously known as zoonotic or rural zoonotic cutaneous leishmaniasis) is, like other forms of cutaneous leishmaniasis, painless when the lesions are uncomplicated. The lesions are often severely inflamed and ulcerated and heal within 2-8 months. Frequently, they are multiple, especially in nonimmune immigrants, becoming confluent and secondarily infected. Such lesions are often slow to heal and may leave large, disfiguring or disabling scars. The incubation period is often less than 4 months.

Cutaneous leishmaniasis caused by L. aethiopica gives rise principally to localized cutaneous nodular lesions; less frequently, it gives rise to oronasal leishmaniasis, which may distort the nostrils and lips, or to diffuse cutaneous leishmaniasis (see section 2.1.4). Most lesions evolve slowly and may spread 
locally. Ulceration is late or absent. Spontaneous healing typically takes place within $2-5$ years.

\subsubsection{Old World mucosal leishmaniasis}

Mucosal lesions of leishmaniasis are rarely seen in the Old World, but any species can cause them. Patients with visceral leishmaniasis or PKDL in India or the Sudan, and also those coinfected with HIV, may develop lesions in the mouth or nose or on the genital mucosa. Lesions of the buccal mucosa or larynx caused by L. infantum, L. major and L. tropica may present in elderly people or people with minor forms of immunosuppression. Laryngeal lesions may become chronic and may be mistaken for cancer.

\subsubsection{Old World diffuse cutaneous leishmaniasis}

Diffuse cutaneous leishmaniasis is caused by L. aethiopica and is characterized by widely disseminated cutaneous macules, papules, nodules or plaques, or by diffuse infiltration of the skin, especially on extensor surfaces of the limbs and on the face, where thickening of the eyebrows and ear lobes may resemble lepromatous leprosy. There is no ulceration. Mucosal involvement is confined to the borders of the nostrils and lips. This disease does not heal spontaneously, and relapses are frequent after treatment (see section 3.2.3). Immunosuppression-associated diffuse cutaneous leishmaniasis caused by other Leishmania species can occur in HIV-coinfected patients and people with other forms of immunosuppression (e.g. transplant recipients). Atypical features such as ulceration can occur.

\subsubsection{New World visceral leishmaniasis}

In the New World, visceral leishmaniasis is endemic or sporadic (see sections 4 and 5). The etiological agent is L. infantum, and the disease is clinically similar to that caused by $L$. infantum in the Old World (see section 2.1.1). Most cases occur in children under 10 years of age, but adults are also frequently affected in foci of recent introduction. PKDL is extremely rare. In Brazil, asymptomatic infections and mild forms of the disease are more frequent than fully manifest visceral leishmaniasis. Longitudinal follow-up has shown that some people remain asymptomatic or recover spontaneously from mild disease, while others with these conditions eventually develop clinical visceral leishmaniasis. Risk factors for progression to visceral leishmaniasis include malnutrition, genetic factors and other infectious diseases. HIV coinfections are increasingly reported. 


\subsubsection{New World cutaneous leishmaniasis}

In the Americas, a wide range of clinical manifestations are caused by multiple and phylogenetically distinct Leishmania species. Although some clinical manifestations are more frequently associated with a particular species or subgenus, none is unique to a species. In addition, a substantial but variable proportion of infections are asymptomatic. The clinical forms include localized, disseminated, diffuse and atypical cutaneous and mucocutaneous leishmaniases. The clinical characteristics and causal species are described below, and the geographical distribution, known and suspected vectors and reservoirs are summarized in section 4.1 .

Localized cutaneous leishmaniasis is caused by multiple species of both the Leishmania and Viannia subgenera, the prevalences of which vary within the region of the Americas. Lesions can occur anywhere on the body but generally originate at the site of inoculation, as a macule followed by a papule that ulcerates and expands to a typical round-to-oval craterform lesion or evolves as a nodular lesion. Lesions can develop weeks, months or even years after infection. Primary lesions may be single or multiple. Lymphatic involvement manifests as lymphadenitis or lymphadenopathy and is common to lesions caused by species of the Viannia subgenus. Lesions caused by L. mexicana often heal spontaneously within 3-4 months, whereas lesions caused by the Viannia subgenus species L. braziliensis, L. panamensis, L. guyanensis and L. peruviana may heal without treatment after 6 months. Secondary cutaneous or mucosal lesions can occur; mucosal disease is most frequently associated with $L$. braziliensis and $L$. panamensis infection but can result from infection by other species.

Cutaneous leishmaniasis caused by L. infantum, the species generally associated with visceral leishmaniasis, is often atypical. Lesions are localized nodules or plaques that fall within the clinical spectrum of lesions caused by dermatotropic New World species. L. infantum cutaneous leishmaniasis is reported mainly in Central America and occurs in areas endemic for visceral leishmaniasis among older children and young adults, whereas visceral leishmaniasis occurs predominantly in children under 5 years of age.

\subsubsection{New World mucocutaneous leishmaniasis}

The term 'mucocutaneous leishmaniasis' is correctly applied only to the New World disease, which is caused mainly by L. braziliensis and L. panamensis, (both species of the subgenus Viannia). Most cases are reported in Bolivia, Brazil and Peru. The salient feature of species that cause mucocutaneous leishmaniasis is that they cause metastasis to the mucosal tissues of the mouth and upper respiratory tract by lymphatic or haematogenous dissemination. Similar conditions caused by other Leishmania species have been reported in immunosuppressed patients. 
Studies in Brazil have shown that mucocutaneous leishmaniasis can present from several months to 20 or more years after a cutaneous lesion. Malnourished young adult male migrants are at special risk. Other risk factors include the site of the primary lesion above the waist, multiple or large primary lesions or delayed healing of the primary cutaneous leishmaniasis. Nasal lesions are always present, with nodules and infiltration of the anterior cartilaginous septum, leading to obstruction of the nostril and, later, perforation of the septum with collapse and broadening of the nose.

The skin of the nose may be thickened, swollen and hyperaemic. In one third of patients, other sites are involved, in order of frequency: the pharynx, palate, larynx, trachea and upper lip. Local lymphadenopathy is frequent. In the final stage, there is severe mutilation, with obstruction and destruction of the nose, pharynx and larynx. Mucocutaneous leishmaniasis almost never heals spontaneously. Secondary bacterial infections are frequent, intercurrent pneumonia being the commonest cause of death.

\subsubsection{New World diffuse cutaneous leishmaniasis}

New World diffuse cutaneous leishmaniasis is clinically and pathologically similar to the Old World form. There are usually no mucosal lesions. The condition does not heal spontaneously. Initially, the disease responds to standard treatment but relapses and becomes unresponsive to further treatment. Diffuse cutaneous leishmaniasis has been associated only with $L$. mexicana and L. amazonensis. An unusual focus occurred in the Dominican Republic.

\subsubsection{Disseminated cutaneous leishmaniasis}

Disseminated cutaneous leishmaniasis presents as extensive, numerous nodular or ulcerated lesions and has been described in association with L. braziliensis, L. panamensis, L. guyanensis and L. amazonensis infections. Over 20 and up to hundreds of cutaneous lesions may occur with or without mucosal involvement. The delayed-type hypersensitivity response to Leishmania antigen and antibody response are intact, and the lesions respond partially to treatment with antimonial medicines and miltefosine.

\subsubsection{Post-kala-azar dermal leishmaniasis}

PKDL occurs in all areas endemic for $L$. donovani but is commonest in East Africa and on the Indian subcontinent, where up to $50 \%$ and $10 \%$ of patients with kala-azar, respectively, develop the condition. The frequency is reported to be declining in India. PKDL usually appears 6 months to 1 or more years after apparent cure of visceral leishmaniasis but may occur earlier or even concurrently with visceral leishmaniasis in the Sudan. There may be no history of previous leishmaniasis. Hypopigmented or erythematous macules on any part of the body may later become papular or nodular and infiltrative, 
especially on the face. The macules are often confused with lesions of vitiligo or leprosy. PKDL may also affect the buccal and genital mucosa and the conjunctiva. PKDL heals spontaneously in a proportion of cases in Africa but rarely, if ever, in patients in India.

In the Sudan, three grades of severity of PKDL have been described:

Grade 1: scattered maculopapular or nodular rash on the face with or without lesions on the upper chest or arms

Grade 2: dense maculopapular or nodular rash covering most of the face and extending to the chest, back, upper arms and legs, with only scattered lesions on the forearms and legs

Grade 3: dense maculopapular or nodular rash covering most parts of the body, including the hands and feet; the mucosa of the lip and palate may be involved.

There is no standard grading system for the severity of PKDL in use on the Indian subcontinent.

\subsubsection{Leishmania and HIV coinfection}

HIV and Leishmania reinforce each other in a detrimental manner. Visceral leishmaniasis is more likely to develop in HIV-infected patients and impairs their response to antiretroviral treatment. In general, patients with HIV-visceral leishmaniasis coinfection present with the manifestations described in section 2.1.1, although splenomegaly is observed less frequently ( $80 \%$ versus $97 \%$ in one series). In profoundly immunosuppressed patients, atypical sites may be infected, including the gastrointestinal tract, peritoneal space, lung, pleural space and skin. Oesophageal involvement can lead to dysphagia and odynophagia, which must be distinguished from other causes of oesophagitis, such as candidiasis.

Tegumentary leishmaniasis in AIDS patients in the New World shows multiple, polymorphic and relapsing lesions. Diffuse cutaneous and PKDL forms associated with visceral leishmaniasis have been reported.

Diagnostic and treatment considerations for HIV-coinfected patients are described in sections 3.1.5 and 3.2.4, respectively.

\subsection{Pathology}

\subsubsection{General pathology}

Various diseases are induced by different Leishmania species, and individual species vary in pathogenicity in different human populations. It is generally accepted that control of Leishmania within the host is mediated by innate and adaptive immune responses. The interplay of Leishmania and human host response is manifest not only in terms of the clinical or subclinical outcome of infection but also the rate of spontaneous healing and recurrent disease. 
Neutrophils are the first cells to confront Leishmania at the site of inoculation by sandflies, and the cells of the innate immune system, including natural killer cells, have been shown to influence the course of infection and disease. Experimental evidence indicates that the pathogenesis of some Leishmania species ( $L$. major) is enhanced by neutrophil intermediation of infection, while neutrophils contribute to protection against others (L. donovani and L. amazonensis).

Either an excess or a deficit of the immune response can lead to chronic, therapeutically challenging disease presentations. Lack of Leishmaniaspecific cell-mediated responsiveness characterizes nonulcerating diffuse cutaneous leishmaniasis; infection-mediated immunosuppression during visceral leishmaniasis leaves the host defenceless against a massive parasite burden, and heightened cell-mediated immune hypersensitivity produces disfiguring chronic mucosal and cutaneous disease. Notwithstanding clinical evidence, the defining role of the immune response was unequivocally established by inverting susceptible and resistant phenotypes in genetically defined experimental models. Selective deletion and replacement of immunocompetent cell populations and, most recently, targeted deletion of the genes coding cell products involved in the immune response have been used to dissect the immunopathogenic and healing responses to experimental infection with L. major and to a lesser extent with other Leishmania species. It is important to note that the strict Th1 and Th2 dichotomy in many experimental murine models does not reflect human disease, in which a mixed picture is often observed.

\subsubsection{Visceral leishmaniasis}

The reticuloendothelial hyperplasia that follows infection with $L$. donovani or $L$. infantum affects the spleen, the liver, the mucosa of the small intestine, the bone marrow, the lymph nodes and the other lymphoid tissues. Many of these cells are heavily parasitized, and lymphocytic infiltration is scanty. In the spleen and other lymphoid organs, there may be atrophy of paracortical areas (white pulp), but plasma cells are numerous. The lifespan of leukocytes and erythrocytes is reduced, causing granulocytopenia and anaemia. Liver function may be normal or altered; later, prothrombin production decreases. Together with thrombocytopenia, the prothrombin depletion may result in severe mucosal haemorrhage. Hypoalbuminaemia is associated with oedema and other features of malnutrition. Diarrhoea may occur as a result of intestinal parasitization and ulceration or secondary enteritis. In the advanced stage, intercurrent infections are frequent, especially pneumonia, dysentery and tuberculosis, and these are common causes of death. 
Hyperglobulinaemia (mainly polyclonal immunoglobulin G) and polyclonal $\mathrm{B}$ cell activation is common in visceral leishmaniasis, but its pathological role is not known. Complement activation may contribute to anaemia; immune complexes are formed, but nephritis is rare. The bone marrow is hypercellular, with erythroid hyperplasia and dyserythropoietic changes. Amastigote forms of Leishmania can be found within bone marrow macrophages and in occasional neutrophil and eosinophil granulocytes.

Human visceral leishmaniasis is associated with mixed Th1 and Th2 responses. In vitro, the lymphoproliferative response is inversely related to disease severity. An absence of lymphocyte proliferation and production of interferon- $\gamma$ in vitro have been associated with progression of $L$. infantum infection to visceral leishmaniasis in recently infected children. Cure following treatment is accompanied by increased interferon- $\gamma$ and interleukin (IL)-12 and decreased IL-10 and transforming growth factor- $\beta$. The number of $\mathrm{CD} 4+\mathrm{CD} 25+\mathrm{T}$ cells is reported to be increased during active visceral leishmaniasis and to decrease at cure. These regulatory $\mathrm{T}$ cells may contribute to the state of immunosuppression characteristic of visceral leishmaniasis.

\subsubsection{Post-kala-azar dermal leishmaniasis}

PKDL is considered to be triggered immunologically and follows apparently successful treatment of visceral leishmaniasis in a proportion of patients (see section 2.1.10). Histologically, the macular and hypopigmented varieties consist of isolated areas, with a granulomatous reaction and few parasites. The more common erythematous and nodular forms show considerable histiocytic infiltration, oedema, proliferation of capillaries and numerous parasites. The inflammatory cells are mainly CD3+, IL-10 is prominent in the lesions, interferon- $\gamma$ is found uniformly, and IL- 4 is present in varying amounts. Diminished expression of interferon- $\gamma$ receptor 1 and tumour necrosing factor (TNF)-R1 and -R2 receptors during PKDL may interfere with an effective host response. IL-10-expressing CD3+CD8+ lymphocytes are prominent, and their level decreases with treatment. Patients with PKDL present raised levels of immunoglobulins G3 and G1 and increased serum levels of IL-10. High serum concentrations of IL-10 during visceral leishmaniasis correlate with subsequent development of PKDL. Antiretroviral treatment during HIV coinfection can lead to PKDL.

\subsubsection{Uncomplicated cutaneous leishmaniasis}

The histological response comprises both the immune cellular response, which reflects the host's immunity and is the basis for classification, and the tissue response, which may reflect the effects of released antigen, as tissue damage is often greater than would be expected from the effects on the host macrophage alone. 
In early forms and in patients with persistently low levels of antibodies, there are large numbers of parasite-laden macrophages, some of which are vacuolated and carry numerous parasites. The infiltrate of lymphocytes and plasma cells increases progressively as the lesion evolves and remains heavy to the end. Elimination of parasites usually follows destruction of the host macrophages, either at the centre of circumscribed clusters in the dermis, with the release of amastigotes, or in macrophages in the subepidermal zone, causing liquefaction of the basal layer and ulceration. Polymorphs are present in the necrotic zone, and lymphocytes are numerous at the periphery. Resolution occurs by replacement of the necrotic centres by Langhans giant cells and a few epithelioid cells.

\section{Tissue response}

During the period of active destruction of parasites, one or more acute changes are usually seen. There is oedema in the superficial dermis and damage to collagen and elastin, with an increase in reticulin, followed by fibrosis. In some cases, there is necrosis of collagen or epidermis, and pseudoepitheliomatous hyperplasia is often severe. At this stage, the small capillaries may show endothelial swelling or proliferation, or there may be vasculitis. In the later, tuberculoid phase, some small vessels may be obliterated. Kerotinocyte death through apoptosis has been implicated in ulcer formation.

Widely variable profiles of Th1 and Th2 cytokines are found in localized cutaneous leishmaniasis lesions and elicited in vitro in response to Leishmania antigens. CD4 and CD8 T cell interferon- $\gamma$ and TNF- $\alpha$ producing lymphocytes, macrophages and $\mathrm{B}$ cells constitute the majority of infiltrating cells. IL-10 and IL-13 have been associated with chronic lesions. IL-4 is rarely consistently detected and, if so, in low concentrations. IL-10 is reported to be produced mainly by monocytes and CD4+CD25 T regulatory cells in lesions caused by $L$. braziliensis and L. guyanensis. The role of T regulatory cells in human leishmaniasis is not yet clear; however, these cells are more frequent in chronic lesions.

\subsubsection{Disseminated cutaneous leishmaniasis}

Leishmania-specific antibodies and a cell-mediated immune response (cutaneous delayed-type hypersensitivity, in vitro cytokine responses) to Leishmania antigens is present but can be weaker than in localized cutaneous leishmaniasis. Mucosal lesions are frequent. Few parasites are observed in biopsy sections. 
Histologically, the lesion is dominated by a heavy lymphocyte infiltrate, giant cells and rare epithelioid and histiocytic cells. Fibrinoid necrosis may be seen, but not caseation. Parasites are few or not visible, but they may be isolated by culture.

\subsubsection{Diffuse cutaneous leishmaniasis}

The histopathology of this condition reflects the absence of cell-mediated immunity and is characterized by an intense dermal infiltration of vacuolated, parasite-laden macrophages ('foam cells'), a scarcity of lymphocytes and absence of necrosis and ulceration. After treatment, the lesions show features of acquired cellular immunity, including lymphocytic infiltrates and diffuse granuloma. Although the internal organs are not affected, treatment is difficult and relapses are common.

Cell-mediated immune responses to Leishmania antigens in vitro are low or absent in diffuse cutaneous leishmaniasis, while responses to unrelated antigens or polyclonal activators are normal. Plasma cells at the site of the lesion are prominent, and a high titre of Leishmania-specific antibodies is found in serum. An absence of cutaneous delayed hypersensitivity to leishmanin skin test antigen and low or absent interferon- $\gamma$ production by peripheral blood mononuclear cells characterize this disease presentation. Parasites are abundant in histopathological tissue sections. High concentrations of TNF- $\alpha$ are found in sera of patients. The outcome of diffuse cutaneous leishmaniasis is often attributed to host factors; however, some studies suggest a role of the infecting organism.

\subsubsection{Mucocutaneous leishmaniasis}

Histological lesions similar to those observed in cutaneous leishmaniasis occur in mucocutaneous disease. Initially, an exudative, nonspecific cellular reaction predominates, with infiltration of lymphocytes, macrophages and plasmocytes, sometimes associated with minor necrotic and granulomatous reactions. Subsequently, a granuloma develops around the necrotic area, with fibrinoid degeneration. Recent evidence indicates that an important aspect of the pathogenesis is acute vasculitis, with coagulative necrosis of the walls of the small blood vessels. At this stage, the lesion can either progress to an epithelioid granuloma (tuberculoid type) organized in tubercles or revert to a cellular exudative reaction.

The presence of immunoglobulins in plasma cells at the site of the lesion has been reported, and it was suggested that the necrosis is caused by accumulated immune complexes. Nonspecific cellular infiltration is often associated with 
ulceration, which, unlike in the cutaneous form, appears to be induced without macrophage necrosis. The histological equivalent of leishmaniasis recidivans is found in patients with mucosal involvement who are resistant to treatment; other lesions in these patients consist only of collagen degeneration without inflammation or attempted repair, which is difficult to explain. The most severe part of the lesion is in the deep nasal mucosa, where amastigotes are present in proliferating vascular endothelium, associated with a heavy perivascular cellular infiltrate and liquefaction of the cartilage. This deep lesion bears little relation to the superficial ulcer.

Marked cutaneous delayed hypersensitivity, exuberant lymphoproliferation and mixed Th1 and Th2 cytokine responses characterize this disease presentation. Interferon- $\gamma$ producing CD4 and CD8 T cells abound in mucosal lesion biopsy samples. Less expression of IL-10 receptor and the anti-inflammatory cytokine IL-10 than in the cutaneous form may contribute to the pronounced proinflammatory response. Parasites are scarce in histopathological tissue sections. TNF- $\alpha$ is present in patient sera and biopsies and elicited in high concentrations in vitro in response to Leishmania antigens. Polymorphism in TNF- $\alpha$ promotor sequences has been associated with mucosal leishmaniasis, and the inhibitor of TNF- $\alpha$ synthesis, pentoxyphylline, has immunomodulatory coadjuvant action in combination with antimonial medicines in the treatment of dermal leishmaniasis.

\subsection{Parasitology}

As mentioned above, the species of Leishmania strongly determines how the disease will evolve. It is essential to know the identity of the parasite(s) in each focus, as this knowledge has implications for epidemiological understanding, control and treatment. Routine identification may be necessary in some circumstances, such as for New World cutaneous leishmaniasis in foci with multiple circulating species.

Unknown stocks (unidentified isolates) should be compared with international reference strains (identified stocks; see also Annex 1), which can be provided by national or international reference laboratories. At present, cultured parasites are required for identification by isoenzyme analysis, which remains the standard reference technique from a taxonomic point of view (see Annex 2). Various molecular techniques are available, generally based on DNA amplification by PCR techniques, followed by sequencing or restriction fragment length polymorphism analysis. These methods can be used directly with clinical samples from patients, reservoir hosts or sandflies. Nevertheless, standardization of the molecular techniques remains a priority. Accurate documentation of the strains is essential (see Annex 1). 


\subsubsection{Identification criteria}

The genus Leishmania is divided into two subgenera on the basis of their development in sandflies. Growth of species of the subgenus Leishmania is restricted to parts of the alimentary tract of the natural vectors anterior to the pylorus at the junction of the midgut and hindgut (suprapylarian development), whereas that of species of the subgenus Viannia occurs in both the midgut and the hindgut (peripylarian development).

Parasite identification at genus level has up to now been based on global taxonomics derived in the 1990s with the isoenzyme technique in comparison with reference strains. Geographically limited studies were conducted by different authors using various molecular approaches. Infraspecific identification depends on the method used, e.g. zymodemes (parasite populations with common isoenzyme patterns identified electrophoretically) or schizodemes (parasite populations defined by shared 'fingerprint patterns' obtained by a technique involving digestion of kinetoplast DNA by restriction enzymes). The results are of practical use in descriptive epidemiology and permit the grouping of parasites into hierarchies that suggest their evolutionary relations (see section 2.3.4).

\subsubsection{Reference strains}

In order to identify leishmanial isolates by isoenzymes, molecular biology and other methods, reference strains are required. These strains are critically important, as they allow identification of the stock. Reference strains must be used systematically for typing. A list of 29 reference strains corresponding to most recognized species is given in Table 2 , which includes recently described taxa.

Table 2.

\section{Leishmania reference strains}

\begin{tabular}{ll}
\hline Species & International code \\
\hline L. (L.) aethiopica & MHOM/ET/72/L 100 \\
\hline L. (L.) amazonensis ${ }^{\mathrm{a}}$ & MHOM/BR/73/M2269 \\
L. (L.) arabica ${ }^{\mathrm{i}}$ & MPSA/SA/83/J1SH220 \\
L. (L.) aristidesi & MORY/PA/69/GML3 \\
L. (L.) donovani & MHOM/IN/80/DD8 \\
L. (L.) garnhami & MHOM/VE/76/JAP78
\end{tabular}


L. (L.) gerbillip

L. (L.) infantum chagasi

L. (L.) infantum

L. (L.) killicki

L. (L.) major

L. (L.) mexicana

L. (L.) pifanoi

L. (L.) tropica

L. (L.) forattiniti

L. (L.) venezuelensis

L. (V.) braziliensis

L. (V.) braziliensis ${ }^{a}$

L. (V.) guyanensis

L. (V.) lainsoni

L. (V.) lindenbergi

L. (V.) panamensis

L. (V.) peruviana

L. (V.) utingensis ${ }^{b}$

L. colombiensis

L. deanei

L. enriettio

L. equatoriensis ${ }^{b}$

L. hertigi
MRHO/CN/60/GERBILLI

MHOM/BR/74/M2682

MHOM/TN/80/IPT1

MHOM/TN/86/LEM904

MHOM/SU/73/5-ASKH

MHOM/BZ/82/BEL21

MHOM/VE/57/LL1

MHOM/SU/74/K27

MDID/BR/77/Conchas

MHOM/VE/00/H17

MHOM/BR/00/LTB300

MHOM/BR/79/M2904

MHOM/GF/79/LEM85

MHOM/BR/81/M6426

MHOM/BR/96/15733

MHOM/PA/71/LS94

MHOM/PE/84/LC39

ITUB/BR/77/M4694

IHAR/CO/85/CL500

MCOE/BR/74/M2674

MCAV/BR/45/L88

$\mathrm{MCHO} / \mathrm{EC} / 82 / \mathrm{Lspl}$

MCOE/PA/65/C8

The taxonomic position of strains with no subgenus designation is under investigation. Updated information on standard strains and strain coding is available on the site of the International Leishmania Network: http://leishnet.net/

a Genome sequenced at the Wellcome Trust Sanger Institute

${ }^{\mathrm{b}}$ No record in humans 
Reference strains and others on which information is published or disseminated are labelled by a four-element code (WHO code), indicating (1) the host from which the strain was isolated, (2) the country in which the infection was acquired (if known with certainty), (3) the year of isolation and (4) the first laboratory designation of the isolate, which should remain a permanent part of the code and not be replaced by a cryobank number. Lists of these codes are given in Annex 1. The scientific name, not the common name, is used for the animal host. The first letter of the first element of the code (the animal from which the strain was isolated) refers to the class to which the animal belongs (e.g. M for Mammalia, I for Insecta). The next three letters represent the generic name for mammals (e.g. MHOM for Homo) and the specific name for sandflies (e.g. IFLA for flaviscutellata). If the identity of the host is unknown at the time of registration, the host code is M000 for a mammal and I000 for an insect vector. In case of duplication, the third letter is modified. Up to 2000, the year of isolation is indicated by the two last numbers of the twentieth century year. For dates since 2000, the four numbers of the isolation year are obligatory, in order to differentiate it from missing data, which are coded as 00 .

\subsubsection{Identification methods}

\section{Isoenzymatic identification}

The most widely used biochemical method is analysis of isoenzymes by electrophoresis (multilocus enzyme electrophoresis). This method remains the baseline of identification, as it is based on a large number of epidemiologically defined isolates in each taxonomic group. Standardized methods have been established in various centres. The main technical limitations are the requirement to isolate parasites in culture and the small number of centres currently using isoenzyme typing. The efficiency of the method is based on the number of enzymatic systems analysed and its reproducibility in different centres (see Table A.1.3 in Annex 1).

\section{Molecular identification}

Faster, more reliable molecular techniques will probably replace multilocus enzyme electrophoresis in the future. These techniques have the advantage that they can be applied directly to biological samples, avoiding parasite culture. Methods based on housekeeping genes allow identification of phylogenetic relations. Techniques based on e.g. sequencing, restriction frangment length polymorphism or single-strand conformation polymorphism are used for the identification of single isolates for molecular tracking and are applicable for outbreak investigations, studies of strain dispersion in different niches or the involvement of reservoirs or vectors in the transmission cycle. 
The main limitations of these techniques are lack of standardization among laboratories and incomplete correlation with the results of isoenzyme typing.

\section{Identification services}

Isolates originate from either research projects or control services and associated clinical, entomological and zoological activities. Before material is sent to a centre for identification, an agreement should be reached on the number of strains that can be handled and on the method of shipment. A formal 'material transfer agreement' should be established between the parties involved. The shipment should be made in accordance with international safety regulations for infective material transport (triple packaging). An accompanying form should be completed for each strain that is sent.

\section{Cryobanks}

Cryobanks should be extensive enough to permit storage of isolates, which can then be made available to research workers (see Annex 2). Operating such banks consumes a great deal of time and resources, and material deposited in them should therefore be well documented. Over the past few years, certain laboratory collections have evolved into biological resource centres, with specific requirements, such as a catalogue for resource access, a quality management system and the traceability of biological material. Material should be requested only for specific purposes, and the requesting laboratory should have the facilities to maintain the stocks for the duration of the study. The identification banks should hold all the WHO reference strains, together with standard strains of newly described taxa. Material can be sent only after signature of a material transfer agreement that defines the conditions of use.

\subsubsection{Taxonomy}

Various classifications have been used for the genus Leishmania. Those proposed between 1916 and 1987 were monothetic Linnean classifications based on a few hierarchical characters. These systems evolved to a classificationdividing the genus Leishmania into two subgenera ${ }^{2}$ : Leishmania, present in both the Old and the New Worlds, and Viannia, restricted to the New World.

Since the 1980s, Adansonian classifications have also been used, which are based on a number of similarly weighted characters, with no hierarchy. The classifications were initially phenetic. Isoenzymes are considered to be different allelic forms of a gene, and enzymatic variation at a given locus can be interpreted as a mutation that occurred during evolution. Subsequently,

2 Lainson R, Shaw JJ. Evolution, classification and geographical distribution. In: Peters W, Killick-Kendrick R, eds. The leishmaniases in biology and medicine. London, Academic Press, 1987:1-120. 
phylogenetic classifications revealed parental relations among the different species of Leishmania, as confirmed by the use of various molecular markers. These methods confirmed the division into two subgenera established by Lainson and Shaw, and the concordance validated the extrinsic and intrinsic identification criteria. The taxonomic position of the species presently described in humans is shown in Table 1, paragraph 2.1.1. A number of undescribed species, some of which cause disease in humans, are under study.

\subsection{Reservoir hosts}

The leishmaniases can be grouped into two broad categories according to the source of human infection: zoonotic leishmaniases, in which the reservoir hosts are wild animals, commensals or domestic animals, and anthroponotic leishmaniases, in which the reservoir host is human. Although each Leishmania species generally falls into one or the other of these categories, occasional exceptions are seen. For example, cutaneous leishmaniasis caused by L. tropica is usually anthroponotic, but in some foci it derives not from humans but from other animals. For several cutaneous leishmaniasis species that are typically zoonotic, humans may constitute an occasional source of infection.

\subsubsection{Definition}

The ecological system in which a Leishmania species is maintained indefinitely is usually composed of one or a small number of sandfly vector species and one or a few vertebrate reservoir host species. Usually, there is one principal reservoir host for a given Leishmania species in a particular focus, but other mammals in the same area may become infected, and these are minor and incidental hosts. Minor hosts may play some role in maintenance of the system and may thereby occasionally bring the parasite from its enzootic focus into closer contact with humans, although such liaisons are infrequent in the transmission of Leishmania. Incidental hosts are mammals which, although infected, play no role in maintenance of the system.

\subsubsection{General aspects of reservoir capacity}

Domestic and sylvatic mammals infected with Leishmania may or may not show obvious signs of infection. Often, there are relatively few amastigotes in the skin or viscera and minimal or no detectable host response. Some mammals, however, such as dogs, which are reservoir hosts of visceral leishmaniasis caused by L. infantum, may eventually be killed by the infection. In dogs, parasites are abundant in the viscera and dermis, from where they are readily picked up by their vectors. The localization of Leishmania in the viscera or dermis of the reservoir host does not necessarily correspond to the 
localization in humans. L. guyanensis, for example, infects internal organs of the sloth with little apparent involvement of the skin, while the same infection in humans is characterized by cutaneous lesions.

\subsubsection{Incrimination of reservoir hosts}

The mere presence of infection in a particular mammal species, even in large numbers, does not necessarily indicate that it is a reservoir host. In order to incriminate a reservoir host formally, it is necessary to demonstrate that the parasite population depends on that particular mammal for its long-term maintenance. This demands extensive ecological studies. In general, full, objective incrimination is not possible, and any conclusions drawn must depend on an accumulation of evidence based on the following criteria:

- A reservoir host is likely to be sufficiently abundant and long-lived to provide a significant food source for sandflies.

- Intense host-sandfly contact is necessary. For example, many Old World principal reservoir hosts are colonial animals that provide suitable habitat conditions (e.g. burrows) for sandfly vectors. A sandfly that has bitten an infective individual reservoir host then has a good chance of biting another subsequently and of transmitting the parasite among the reservoir host population.

- The proportion of individuals that become infected during their lifetime is usually considerable and may exceed $20 \%$, although the prevalence can vary greatly with season.

- The course of infection in a reservoir host should be long enough and the infection should be sufficiently nonpathogenic to allow the parasites to survive any nontransmission season.

- Parasites should be available in the skin or the blood in sufficient numbers to be taken up by a sandfly (see section 2.5.3).

The parasites in reservoir hosts must be the same as those in humans, and formal identification of the parasites is therefore necessary (see section 2.3). Although molecular methods may be useful for examining large numbers of individual animals, isolation of parasites and formal identification are essential for qualitative description of the system.

The status of some hosts has not been fully established, notably that of foxes, jackals, badgers, rats and cats in the maintenance of $L$. infantum foci. There is growing evidence that the domestic cat may be involved in the maintenance of $L$. infantum and that the dog may be important in cutaneous leishmaniasis in South America. It is important that numerous specimens of the supposed reservoir hosts be examined, that their bionomics be understood and that the 
parasites be isolated and formally identified. Accurate identification of reservoir hosts is essential, and the advice of specialists from an appropriate zoological centre of expertise should be sought.

\subsubsection{Humans as reservoir hosts}

Human beings are directly involved as a principal reservoir host in two forms of the disease: visceral leishmaniasis caused by $L$. donovani and cutaneous leishmaniasis caused by L. tropica. Humans have also played a reservoir role in some outbreaks caused by L. braziliensis, L. guyanensis and L. panamensis. The role of asymptomatically infected individuals in the transmission cycle is currently unknown. HIV-coinfected patients are known to be highly infectious to sandflies and may play a role in transmission in some areas. Of the forms of leishmaniasis caused by L. donovani, visceral leishmaniasis and PKDL are sources of infection for sandflies, and cases should be actively sought and treated. The same is true of recurrent forms of cutaneous leishmaniasis caused by L. tropica. In addition, it is possible that humans can act as sources of human infection with $L$. major and in a number of strictly cutaneous forms caused by L. infantum, because of the lingering nature of the lesions. Several Leishmania species can coexist in a single focus, causing clinical forms that seem identical but occur in different epidemiological cycles. In the Arabian peninsula, for example, L. donovani and L. infantum are found together in foci, the former only in humans, the latter in humans and dogs. This highlights the need for exact identification of the parasites (see section 2.3.3).

\subsubsection{Domestic and peridomestic animal reservoir hosts}

Dogs are the principal reservoir hosts of $L$. infantum. They have also been found to be infected with other Leishmania spp., and their role in these infections is probably more than incidental. In the wide geographical range of L. infantum, there are many contrasting situations, depending on whether the dogs are domestic, stray or feral and on the animals' place in society. The discovery in both the Old World and the New World of foci of canine leishmaniasis without notified human cases shows how widespread the parasite is. This is a result not only of animal movement but also of the great versatility of $L$. infantum, which can be transmitted by vectors of different species, subgenera and even genera. Naturally infected asymptomatic dogs have been demonstrated to be easily infective to sandflies under experimental conditions (xenodiagnosis). Therefore, the role they may play in the cycle should not be underestimated, as more than $50 \%$ of all infected dogs are asymptomatic carriers. Some transmission occurs directly from dog to dog, without sandflies; the significance of this finding remains to be determined. 
Dogs, horses, donkeys and mules have been found to be infected in a number of American foci of L. braziliensis. Dogs have also been found to be infected with $L$. panamensis and L. peruviana. The possible role of these animals as a source of infection should not be overlooked. Canine leishmaniasis caused by $L$. infantum has occurred in kennelled foxhounds in several eastern states of the USA with, currently, no reports of human infection. A suspected vector (Lutzomyia shannoni) occurs in the USA, which has been shown experimentally to support the growth of $L$. infantum, and promastigotes have been found in wild-caught specimens. Vector-borne transmission was not, however, demonstrated in the foxhound outbreak.

\subsubsection{Wild reservoir hosts of the Old World}

\section{Non-domestic Canidae}

A number of wild Canidae-fox (Vulpes spp.), jackal (Canis aureus), wolf (Canis lupus) and the raccoon-dog (Nyctereutes procyonoides) - have been found to be infected with L. infantum in both the Old and the New World. The role of these animals as reservoir hosts has been suggested but is not fully established.

\section{Rodents and others}

The reservoir structure varies between foci and must be carefully determined before control measures are instituted. Rhombomys opimus, the great gerbil, is the primary reservoir host of L. major in the arid regions of Central Asia. Colonies of this species are easily detected over large areas by the use of high-resolution remote sensing images in conjunction with observations on the ground. The parasites in R. opimus are not all infective for humans (e.g. L. gerbilli), so careful identification of parasites is required for each location prior to control operations.

Psammomys obesus, the main reservoir host of L. major in West Asia and North Africa, feeds almost exclusively on the leaves and stems of plants of the family Chenopodiaceae. In arid zones, most species of Chenopodiaceae grow in salty ground and dry river valleys. Psammomys obesus is largely confined to these biotopes but can also be found in fields of cereals, earth banks or fallow ground, where it feeds on Chenopodiaceae that are cultivated (Atriplex spp.) or occur as weeds (Anabasis articulata).

Several other species of gerbil, Meriones spp. and Tatera indica and also Nesokia indica with different ecological and ethological characteristics are involved in the maintenance of L. major, indicating again the importance of accurate identification and good ecological analysis as a prerequisite to any intervention. In semi-arid zones in the Maghreb, Meriones shawi lives on 
cereals and vegetables. In sub-Saharan oases, the same species behaves quite differently, feeding on wastes of various kinds, including faeces when in close (peridomestic) contact with humans, which explains how serious epidemics have arisen in such areas. Mastomys spp. and Tatera spp. are the suspected reservoir hosts of cutaneous leishmaniasis in Senegal and the Sudan.

\section{Hyraxes}

Two species of hyrax, Procavia capensis and Heterohyrax brucei, are the reservoir hosts of L. aethiopica in East Africa. One is the suspected reservoir host for an unnamed Leishmania species in Namibia and for L. tropica in northern Israel and, possibly, Saudi Arabia.

\subsubsection{Wild reservoir hosts of the New World}

\section{Non-domestic Canidae}

The crab-eating fox, Cerdocyon thous, is commonly infected with L. infantum. This animal frequently visits villages and may acquire its infection from village dogs. Its role in the ecological system that maintains $L$. infantum, as a minor reservoir host, is still being investigated.

\section{Sloths}

Several species of sloth are important reservoir hosts of various Leishmania species. Choloepus didactylus is a major reservoir host of L. guyanensis in Brazil and in French Guiana; it maintains the zoonosis in the forest canopy. The same species is a reservoir host for $L$. shawi in the Amazon region. Choloepus hoffmani is the primary reservoir host of L. panamensis in Brazil, Colombia, Costa Rica and Panama; Bradypus griseus is a reservoir host of L. panamensis in Costa Rica and Panama; B. infuscatus has also been reported to be infected by L. panamensis, but its importance in the zoonotic cycle is not clear. In areas of Panama, $19.3 \%$ of C. hoffmani were found to be infected; the parasite was present in skin, blood, bone marrow, liver and spleen.

\section{Lesser anteater}

Tamandua tetradactyla, which has arboreal habits, is the principal reservoir host in the transmission cycle of L. guyanensis in Brazil. It has been suggested that this nomadic animal is responsible for dispersal of the parasite.

\section{Opossums}

Didelphis marsupialis is a secondary reservoir host for L. guyanensis and $L$. infantum. It has been suggested that it is a primary reservoir host in disturbed primary forest $(61.9 \%$ of specimens were infected in the Amazonas 
State of Brazil), but this is not the case in the natural zoonotic cycle. It is also a secondary host for L. amazonensis and was found to be infected with Leishmania spp. in a site endemic for visceral leishmaniasis in Colombia. Similarly, in Brazil, D. albiventris was found to be infected with L. peruviana and a Leishmania provisionally identified as L. infantum.

\section{Procyonids}

Potos flavus, Nasua nasua and Bassaricyon gabbii have been reported occasionally to be infected with $L$. panamensis.

\section{Rodents}

Proechimys guyanensis and Proechimys cuvieri are incidental hosts of $L$. guyanensis and primary reservoir hosts of $L$. amazonensis. Several rodent species such as Oryzomys, Nectomys and Dasyprocta can act as secondary hosts of $L$. amazonensis, sometimes with infections of apparently normal skin. In Brazil, spiny rats (Proechimys spp.) infected with L. amazonensis have been found in pine plantations. Many species of ground rodent are involved in the transmission cycle of L. mexicana. Ototylomys spp. are the primary host in Belize and in the Yucatan peninsula of Mexico. Heteromys, Nyctomys and Sigmodon spp. are secondary hosts.

Leishmania braziliensis has been isolated from rodents (Akodon, Bolomys, Nectomys, Rattus) and has been identified by molecular methods in a number of other small ground-inhabiting mammals, including marsupials, in various Brazilian states. This finding shows that a mosaic of small mammals are sources of infection. The incidences of infection found by PCR indicate that the major source of infection depends on the ecological niche. There is no evidence of an arboreal enzootic cycle for L. braziliensis, and all the proven vectors have been found at ground level in endemic areas.

\subsection{Vectors}

\subsubsection{Taxonomy}

Although there is no universal agreement on the generic and higher classification of sandflies, the position of the Old World species is generally accepted and is less controversial than that of the species of the New World. For many years, only three Old World genera were recognized: Phlebotomus, Sergentomyia and Chinius. A few others have been, or are about to be, named but they are of no known medical importance. For Neotropical sandflies, most workers follow the classification of Lewis, with revisions by Young and Duncan, recognizing three genera, 15 subgenera and 11 species groups. For decades, this system has been criticized because it does not reveal 
evolutionary relations between species, particularly of the important genus Lutzomyia $^{3}$. Several revisions have been proposed, but none has been universally accepted. The two most recent and comprehensive are those of Galati $^{4}$, in which, in her second revision, the 464 species of Neotropical sandflies are classified into 22 genera, 20 subgenera, 3 species groups and 28 series. While this classification is an important advance, it has not yet been fully accepted, and further changes are expected. One difficulty is that the females of one fifth of the Latin America species are undescribed.

Although human-biting sandflies occur in some other genera, the only proven vectors of human disease are species and subspecies of Phlebotomus in the Old World and Lutzomyia (sensu Young) in the New World (see section 2.5.4). Suggestions that leishmaniasis may be transmitted by the bite of haematophagous invertebrates other than sandflies (e.g. fleas, ticks) are not supported by convincing experimental evidence.

Questions about the status of some species that are proven vectors are being resolved by identification criteria based on new morphological characters and molecular biology techniques (see section 2.5.2). The best-studied species is Lu. longipalpis, Brazilian populations of which are now recognized as closely related forms. Phlebotomus argentipes, the vector of visceral leishmaniasis in the north-east Indian subcontinent, occurs in many Asian countries where leishmaniasis is absent; from differences in morphology and cuticular hydrocarbons between geographically defined populations, it has been suggested that this species is composed of two or more populations, possibly sibling species, with different vectorial capacities. New studies of the morphology of the spermathecal ducts suggest that previously recognized subspecies of $P$. major, vectors of Old World zoonotic visceral leishmaniasis, are allopatric species (P. major, P. neglectus, P. syriacus, $P$. wui and, possibly, P. krimensis).

\subsubsection{Identification criteria}

Morphological characteristics remain the most inexpensive, widely used, practical means of distinguishing species of sandfly. Many, but not all, males can be identified by their morphology alone, but it is often more difficult to identify females, some of which are proven vectors. One advance is recognition of females of the Old World subgenus Larroussius, including important vectors, from the morphology of the distal portion of the spermathecal ducts. Another is the identification of females from the armature in the genital

\footnotetext{
3 Young, D.G. and M.A. Duncan, 1994. Guide to the Identification and Geographic distribution of Lutzomyia sand flies in Mexico, the West Indies, Central and South America (Diptera: Psychodidae). Mem.American Entomol. Inst., 54: 1-881

4 Galati, 2003 E.A.B. Galati, Classificação de Phlebotominae. In: E.F. Rangel and R. Lainson, Editors, Flebotomíneos do Brasil, FIOCRUZ, Rio de Janeiro (2003), pp. p.23-51.
} 
atrium. While this method cannot be used for all groups, some closely related species of Phlebotomus and of Lutzomyia can be separated by this feature.

In the past, gas chromatography of cuticular hydrocarbons and isoenzyme electrophoresis were helpful for identifying morphologically indistinguishable females of related species. Now, however, these techniques have been superseded by DNA analysis, which allows measurement of genetic differences between populations of closely related species and reveals evolutionary relationships. These new methods do not solve all the problems of taxonomy: judgement is still required to decide whether morphologically similar sandflies warrant recognition as different species.

\subsubsection{Biology}

The biology of each species of sandfly is unique and complex, covering all aspects of reproduction, feeding, dispersal and other activities that have a direct bearing on the epidemiology of leishmaniasis and vector control.

\section{Preimagimal stages}

The life cycle of a sandfly is shown schematically in Figure 2. The eggs, larvae and pupae develop in moist microhabitats rich in organic matter; they are not aquatic. Precise periods for the development of the stages cannot be given because they are affected by ambient temperature, low temperatures lengthening and high temperatures shortening the times. In the laboratory, eggs usually hatch within 7-10 days. Larval development takes at least 3 weeks before pupation. Adult flies emerge from the pupae after 10 days, with males predominating at the beginning. Palaearctic species overwinter as diapausing fourth-instar larvae, whereas in warmer, wetter habitats, diapause occurs in the egg stage. Preimaginal stages are notoriously difficult to find in nature, and the breeding sites of most vectors are unknown. In the Neotropics, some evidence suggests that the open forest floor serves, in a general way, as a common breeding site for forest-dwelling species. In Venezuela, larvae of Lu. youngi have been found in coffee plantations, indicating that the breeding sites of some vectors are not adversely affected by changes in the forest habitat. $P$. argentipes breeds in cattle sheds, and eggs are laid above the water line during seasonal floods in India. In deserts, $P$. papatasi breeds in gerbil burrows.

\section{Mating behaviour}

Some or all Neotropical sandflies recognize mates from pheromones and 'songs' produced by the male by vibrating his wings. Similar pheromones and 'songs' have not been demonstrated for Phlebotomus species, but males of one species of this genus $(P$. duboscqi) mount a female without 
copulation, after which the female accepts mating. Males of some species (Lu. longipalpis, Lu. migonei and P. argentipes) wait on the host for females coming to feed and then court the female, a behaviour described as 'lekking'. Males of other species (e.g. P. ariasi) may never be seen on the host but mate after the female has engorged. Two Neotropical species (Lu. mamedei and Lu. maruaga) are parthenogenetic and autogenous.

Figure 2.

Schematic life cycle of a phlebotomine sandfly (not to scale)

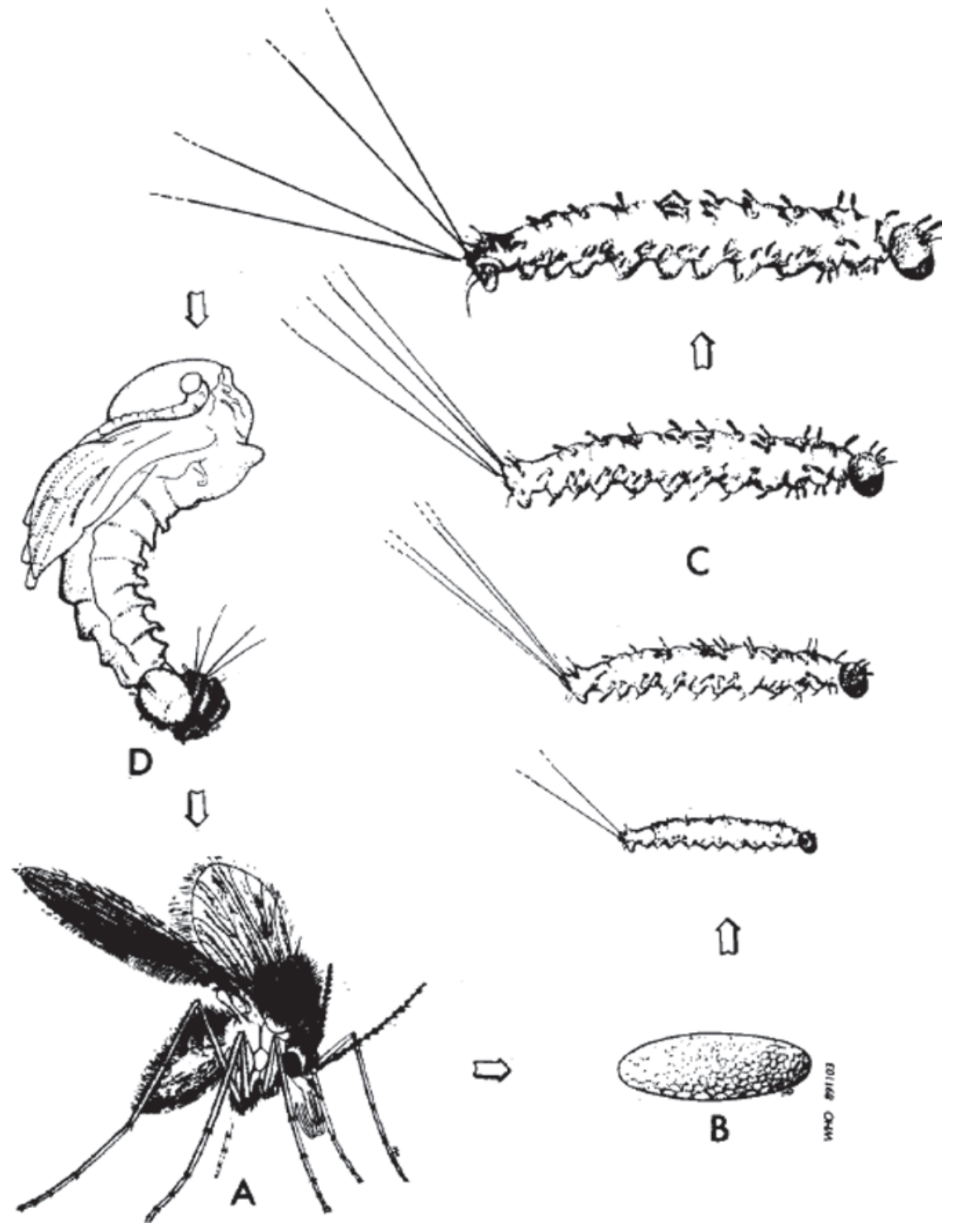




\section{Feeding}

Male sandflies are occasionally seen with blood in the stomach, but, as their mouth parts are too poorly developed to pierce skin, it is assumed the blood was taken from a skin sore. They play no part in transmission. Female sandflies of almost all species require a blood-meal for egg development. Autogeny (development of eggs without a blood-meal) occurs in a few for the first oviposition; thereafter, a blood-meal is essential for further egg batches. For females of species that are not autogenous, it is possible to determine whether they have fed previously by examining the ovarioles for follicular relics (the Polovodova ovarian age grading technique). These observations can provide information on the physiological age of the sandfly population at different times of the year and indicate the best time to search for naturally infected flies, when the highest proportion of females are parous and have, therefore, fed on blood at least once.

The preferences of vectors for different vertebrates vary according to species and to the availability of hosts. During feeding, saliva and parasitic proteophosphoglycans are introduced into the host, which are believed to play a part in the establishment of Leishmania in the skin of the vertebrate. Large numbers of uninfected bites, however, give rise to increasing titres of antibodies to salivary proteins that may alter the course of subsequent infection.

Sugar meals serve as energy sources for sandflies and are important in the development of parasites within the gut. The discovery that both male and female sandflies of many species (e.g. P. ariasi, P. perniciosus, $P$. perfiliewi, Lu. longipalpis, Lu. peruensis) feed on aphid honeydew resolved a longstanding question about the origin of sandfly sugar meals. At least one other species ( $P$. papatasi) also pierces plants for sap, and, while floral nectar has not been demonstrated in wild-caught flies, Lu. longipalpis feeds on flowers in the laboratory. The relative importance of different sugars in the life cycles of Leishmania spp. in the vector is unknown.

\section{Resting sites}

During the day, sandflies rest in comparatively cool, humid niches, including bedrooms; latrines; cellars; stables; caves; fissures in walls, rocks or soil; dense vegetation; tree holes and buttresses; burrows of rodents and other mammals; birds' nests; and termitaria. Depending on the habits of the different species, these are the places where sandflies are most easily collected. Females of many species are predominantly exophagic (biting outdoors) and exophilic (resting outdoors during maturation of eggs) and cannot be controlled by spraying internal walls of habitations with insecticide. In contrast, species that are endophilic (resting indoors during maturation of eggs) can be attacked in this way. 


\section{Oviposition}

From studies of colonized sandflies, it is known that the time from a bloodmeal to oviposition varies with the species and the ambient temperature. The time in nature is known for only one species $(P$. ariasi): 6 days in a markrelease-recapture study of engorged females. The number of eggs deposited depends on the size of the blood-meal taken and may be as many as 200 . Females of some species are gonotrophically concordant (the ovaries develop as a single blood-meal is digested, and the female does not feed a second time during the oviposition cycle, e.g. P. perniciosus, P. ariasi, P. orientalis), whereas females of other species are gonotrophically discordant (no relation between digestion of a blood-meal and development of eggs, and more than one blood-meal may be taken during a single oviposition cycle, e.g. $L u$. longipalpis, $P$. argentipes, $P$. papatasi). In dissections of wild-caught gonotrophically discordant females, two blood-meals in different stages of digestion are commonly seen, and blood-meal analyses reveal a proportion of mixed feeds. These differences presumably affect the competence of vectors, in that, all other factors being equal, gonotrophically discordant vectors have more frequent contact with vertebrate hosts than gonotrophically concordant species.

\section{Life expectancy}

In a mark-release-recapture study of $P$. arias $i$ in France, flies were recaptured up to 29 days after release. Although it is not possible to calculate the life expectancy, as the flies were of unknown age when marked, this observation suggests that 1.54 ovarian cycles ( 9 days), estimated by examining the follicular relics in the ovaries of more than 10000 females of the same species, is an underestimate. There is no information on the life expectancy of wild populations of any other sandfly. Data obtained in the laboratory are unlikely to reflect the actual time in nature.

\section{Flight speed and dispersion}

The flight speed of phlebotomines is in the order of $1 \mathrm{~m} / \mathrm{s}$, considerably less than that of mosquitoes. Sandflies are unable to fly at wind speeds above this rate, which limits their distance of dispersal. As wind speeds increase with height, the flies tend to fly close to the ground. The distance of dispersal indicates the optimum size necessary for a buffer zone around a settlement or village. Evidence from mark- release-recapture studies indicates that forest species disperse shorter distances than peridomestic species. Often, however, the distance is underestimated in these studies because of limitations in the number of sandflies released and the size of the team engaged in recapture. The peridomestic species $P$. ariasi commonly moves more than $1 \mathrm{~km}$ in a 
night and may disperse over $2 \mathrm{~km}$ : $P$. papatasi has been recaptured $1.5 \mathrm{~km}$ from the release point. In contrast, Neotropical forest species appear seldom to disperse more than $1 \mathrm{~km}$.

\section{Population dynamics}

Sampling of a population of a sandfly species must be planned carefully and carried out over extended periods with uniform trapping methods. Catches are influenced by environmental conditions, including wind, temperature, rain, relative humidity, atmospheric pressure and moonlight. These factors can alter the yields of traps placed in sheltered habitats from that of traps placed in open areas. The relative abundance of species cannot be determined from their proportion in the capture because different species may react differently to traps. Sticky traps (paper treated with castor oil) of a standard size with both sides of the paper uncovered are considered to be the least biased sampling method. In one study of $P$. argentipes in India, several sampling methods were carefully compared: hand capture proved to be the most reliable. Endophilic species can be collected early in the morning on cotton sheets on the floor of a room 30 min after spraying with a short-acting insecticide (e.g. a pyrethrum spray).

\subsubsection{Incrimination of vectors}

For several reasons, most sandfly species play no part in the transmission of leishmaniasis: they may never bite humans; their distribution may be different from that of a reservoir host; their feeding preferences may not include a reservoir host; or they may be unable to support the development of the Leishmania (see point 5 below). Of approximately 800 species of sandflies, only 93 are proven or probable vectors of Leishmania (see Tables 4 and 5, paragraph 4.1); however, more vector species are constantly being added to the list. In some foci, the vector or vectors are unknown. Progress in identifying vectors is often slow due to the difficulty of finding naturally infected flies in the wild and the lack of suitably trained, experienced staff. Major advances in the detection of parasites in vivo have been made with new techniques, such as sequential staining of gut smears with labelled monoclonal antibodies and use of DNA probes on squash blots of whole flies or on parasites from dissected guts.

The generally accepted criteria for incriminating Leishmania vectors are:

(1) The vector must be anthropophilic. The usual way of determining which species are anthropophilic is to catch sandflies at night as they come to bite humans. Another approach is to identify fresh blood-meals in female flies collected while they are resting in houses. A dipstick ELISA test for field use has been developed, and a field kit is being designed. 
(2) The vector must bite the reservoir host(s). Most leishmaniases are zoonoses, and confirmation that a suspect vector feeds on the reservoir host is strong supporting evidence of a vectorial role. Conversely, if females are seldom or never found to be attracted to a reservoir host, that is evidence of a negative role. The feeding preferences of sandflies are best determined by comparing the catches in traps baited with different animals. The Disney trap is useful for small animals, whereas tent traps are used for large animals. Identification of blood-meals shows the range of available animals on which a species feeds in nature (but does not indicate preferences).

(3) The vector must be infected in nature with the same Leishmania as occurs in humans. Establishing this requires identification of the parasites from the sandflies and comparison with isolates from patients. The usual method is to dissect female flies and to identify parasites from infected midguts (see section 2.3). Isoenzymes or DNA of the cultured parasites are then compared with those of isolates from humans. Primary isolates of some species of Leishmania, however, are difficult or impossible to grow, and contamination of cultures from sandflies by bacteria or fungi results in loss of the isolates. An alternative to culturing directly from flies is to inoculate the promastigotes from an infected gut into hamsters or susceptible inbred mice. The practice of grinding up batches of flies is not recommended, as it is rarely possible to be sure that the batch contains only a single species. When the target Leishmania is known, promastigotes can be identified directly from sandflies by DNA amplification and probes or monoclonal antibodies. Both tests can be done without first isolating the parasite in culture. As the parasites may be present in the bloodmeal of a sandfly that is not a vector, positive results from fed flies can be misleading. The most meaningful results are from females with no undigested blood in the stomach.

(4) The vector must support flourishing growth of the parasite it transmits. When sandflies are infected with the Leishmania they transmit in nature, the parasite persists even after the blood-meal has been digested and passed in the faeces. Observations on the intensity of infection in naturally infected sandflies can be supplemented by examination of sandflies infected experimentally by feeding on an infected animal (e.g. dog, hamster, gerbil, sloth). After feeding, the flies are maintained on sugar and are examined 7-10 days later. Ideally, colonized sandflies are used, but, if this is not possible, observations can be made with flies caught in the wild. Some Lutzomyia spp., however, have a wide vectorial range and have been shown experimentally to be capable of supporting growth of Old World species as well as parasites of the L. mexicana group, of which thay are not natural vectors. 
(5) The vector must be able to transmit the parasite by bite. It is technically difficult to establish laboratory colonies of some species of sandflies and to persuade the females to bite twice, thus achieving transmission. On these grounds, verification of this criterion may not be practicable, although it remains highly desirable.

\section{Practical considerations}

The main limitations on research into incrimination of vectors are lack of expertise, financial support and facilities. It is therefore seldom possible to undertake extensive investigations to provide incontestable evidence. In practice, the first steps are to make an inventory of species and find out which bite humans. The results of such preliminary steps and knowledge about vectors in other foci will usually direct suspicion towards one or two species. Samples of suspected vectors should be collected and dissected when parous flies form a high proportion of the target population, not when the population is at its highest and largely composed of nulliparous females that have never taken a blood-meal. The parasites must then be compared with isolates from patients in order to incriminate the vector(s) (see section 2.3).

\subsubsection{Vector competence}

Many species of sandfly are unable to support the growth of Leishmania. Vectors can be divided into specific vectors, which support the growth of only one species of Leishmania, and permissive vectors, which support the growth of more than one species. The main factors that influence the ability of a given species of sandfly to act as a vector are, first, resistance by the parasite to the action of digestive enzymes produced in the midgut of the fly; secondly, the presence on the inner surface of the gut of the vector of the appropriate lipophosphoglycan-binding sites that match the lipophosphoglycan on the surface of the promastigotes, enabling them to attach to the inner surface of the sandfly gut; and, thirdly, completion of the development of the parasite to produce metacyclic promastigotes, the only form of the parasite that can survive in the vertebrate host. Infected sandflies have difficulty in taking a blood-meal and frequently probe several times and take smaller-thannormal blood-meals. This is thought to be because of damage caused by the parasite to the stomodeal valve at the anterior end of the midgut from which metacyclic promastigotes are assumed to be regurgitated into the skin of the vertebrate. This, however, may not be the only mechanism of transmission. Metacyclic promastigotes are frequently seen in the proboscis of infected females, and it is reasonable to suppose they would be deposited in the skin of the vertebrate at a blood-meal. Another possibility is deposition of metacyclics, which have been seen in the salivary glands of wild-caught Neotropical species of sandfly (L. naiffi in Lu. umbratilis and Lu. squamiventris) and 
in P. duboscqi experimentally infected with L. tropica. The time from an infecting blood-meal until a female can transmit leishmaniasis by bite is probably 1-3 weeks, although the precise period is not known for any species of Leishmania and undoubtedly varies according to the gonotrophic cycles of the different vector species, the ambient temperature and, perhaps, the sugars taken by the females.

\subsection{Epidemiological aspects}

The epidemiology of leishmaniasis depends on the characteristics of the parasite species, the local ecological characteristics of the transmission sites, the current and past exposure of the human population to the parasite and widely varying human behaviour. (The burden of leishmaniasis is discussed in section 4.2.)

Leishmaniasis can be transmitted by shared syringes among intravenous drug users, by blood transfusion and congenitally from mother to infant, but these modes of transmission are rarer than vector-borne transmission. This section focuses on the epidemiology of leishmaniasis in locations with vector-borne transmission.

The age range most affected depends on the parasite species and the history of population exposure. For example, in endemic foci where the causative parasite is $L$. infantum, the median age of clinical visceral leishmaniasis patients tends to be younger (usually $<5$ years) than in endemic foci of L. donovani (median age, 13-23 years in various sites in Asia and Africa). In general, in populations in which substantial levels of transmission have been sustained over periods of years, a large proportion of the adult population will have acquired immunity to the parasite, as indicated by a high prevalence of positive leishmanin skin test results, the prevalence often rising with increasing age (see section 2.6.10 for epidemiological surveys with the leishmanin skin test). Naive or immunosuppressed adults who enter an endemic area are nevertheless at risk for the disease, even if the circulating parasite is L. infantum.

Leishmania and HIV coinfection was first observed in the mid-1980s in patients with visceral leishmaniasis in southern Europe and has since appeared in one third of the endemic countries. Visceral leishmaniasis in coinfected patients cannot be cured, and those with CD $4+$ counts $<200$ cells/ $\mu 1$ typically relapse more and more frequently, until they become unresponsive to all medicines used. They characteristically have very high parasite loads and thus contribute to increased spread of the infection, which may include the spread of drug-resistant strains. In addition, these patients have been proven to be highly infective to sandflies, and their presence in substantial numbers may increase the effective infection reservoir. 
Although health-care facilities in many regions report more male than female cases, the sex ratio can be accurately ascertained only in community-based studies, as data from facilities reflect any disparities in access to health care (see section 3.7).

The conditions under which humans become infected vary considerably with time and place. In many disease foci, leishmaniasis is a zoonosis, and the 'intrusion' of humans into sylvatic cycles results in a higher risk for infection. In other cases, transmission is anthroponotic, as for visceral leishmaniasis on the Indian subcontinent and during epidemic spread in East Africa. In some parts of both the Old and the New World, transmission can be domestic or peridomestic. Changes in the environment have a strong influence on the epidemiology of leishmaniasis, and it has been suggested that the distribution and burden of the disease will be affected by climate change caused by global warming. Geographical information systems are valuable for basic and operational research on the epidemiology of leishmaniasis, and their use should be extended.

\subsubsection{Major foci and human behaviour}

\section{Old World}

In the Mediterranean Basin, visceral leishmaniasis is a zoonosis caused by $L$. infantum. It occurs in rural areas, in villages in mountainous regions and also in some periurban areas. Houses and their surroundings form the microfoci where human-fly contact occurs and where transmission takes place. Many people in this region have dogs - the proven reservoir host of L. infantum - as well as other animals that attract sandflies into their houses and therefore favour transmission to humans. Zoonotic L. infantum transmission foci are also found in Afghanistan, the Islamic Republic of Iran, Pakistan and Central Asia.

In anthroponotic foci in Bangladesh, India and Nepal, the conditions that favour transmission of visceral leishmaniasis are generally rural areas at less than $600 \mathrm{~m}$ above sea level, a heavy annual rainfall, a mean humidity above $70 \%$, a maximum temperature of $38^{\circ} \mathrm{C}$ and a minimum temperature of $15^{\circ}$ $\mathrm{C}$, with a diurnal variation of less than $7^{\circ} \mathrm{C}$, abundant vegetation, subsoil water and alluvial soil. The disease occurs in agricultural villages where houses are frequently constructed with mud walls and earthen floors, and cattle and other livestock are kept close to human dwellings. Spatial analyses demonstrate significant clustering of visceral leishmaniasis cases, both at the household level and on a larger scale. The proven vector, $P$. argentipes, is found in higher density in cattle sheds than human dwellings but takes bloodmeals opportunistically from both bovines and humans. Human behaviour, 
such as sleeping outside or on the ground, may increase risk, while the use of bednets tends to be associated with lower risk.

In East Africa, visceral leishmaniasis occurs in two distinct ecological settings, the Acacia-Balanites savannah regions in the north, where P. orientalis is the major vector, and the savanna and forest areas in the south, where $P$. martini and $P$. celiae are found in association with Macrotermes termite mounds. While sporadic sylvatic zoonotic visceral leishmaniasis transmission is well recognized, sustained peridomestic and domestic cycles in villages have also been demonstrated. Large outbreaks, such as those that occurred during the civil war in southern Sudan in the 1980s and 1990s, are thought to be due to anthroponotic transmission in conditions of massive population displacement, destruction of health-care infrastructure and increased incidences of other diseases. Seasonal labour movements may also spread the disease, with the return of migrants to nonendemic areas, as appears to have occurred in the highlands of Ethiopia in the 2000s. Specific risk factors include the presence of cattle and the person's age and genetic background. Behaviour such as sleeping outside under acacia trees and living in houses constructed of grassy material appears to increase risk for the disease. In areas where $P$. martini and $P$. celiae are the predominant vectors, the proximity of human dwellings to termite mounds increases risk. Although L. donovani infection has been demonstrated in dogs in several foci, their importance in the transmission cycle is uncertain.

In the Old World, L. major and L. aethiopica cause zoonotic cutaneous leishmaniasis. The risk may be increased when agricultural projects are launched and irrigation systems extended. These man-made ecological changes are accompanied by the intrusion of large numbers of nonimmune immigrants into an existing sylvatic cycle of leishmaniasis. Transmission to humans is favoured by the practice of sleeping outside without a bednet during the hot season, the transmission period. The risk for infection is also increased by activities such as tourism and pilgrimages to endemic areas. In foci of cutaneous leishmaniasis caused by L. aethiopica in the highlands of Ethiopia and other places in East Africa, increased human-fly contact occurs in villages built on rock hills or river banks, which are the natural habitat of hyraxes (reservoir hosts) and of $P$. pedifer. Cases have also been reported in and near urban centres, including Addis Ababa.

In contrast, although infections in dogs and other animals have been documented, cutaneous leishmaniasis caused by L. tropica occurs in a predominantly anthroponotic cycle. Anthroponotic cutaneous leishmaniasis is an urban and periurban disease, and shows patterns of spatial clustering similar to those seen for anthroponotic visceral leishmaniasis in South Asia. The disease is characterized by large outbreaks in densely populated cities, 
especially in the setting of war and large-scale population migration, for example, in Kabul in the 1990s and early 2000s.

Recently, increasing numbers of cases of cutaneous leishmaniasis have been reported from West Africa. An urban outbreak occurred in Ougadougou, Burkina Faso, in which HIV coinfection was also reported.

\section{New World}

In the New World, visceral leishmaniasis results from zoonotic transmission of $L$. infantum, and its epidemiology resembles that of the disease in the Mediterranean Basin. The habit of keeping dogs and other domestic animals inside the house is thought to promote human infection, as dogs are the $L$. infantum reservoir hosts and also attract sandflies, especially $L u$. longipalpis. The location of hen houses close to the main habitation may be another important risk factor, as they constitute a major feeding and resting site for $\mathrm{Lu}$. longipalpis, thus increasing sandfly contact with dogs and humans. Furthermore, chickens attract wild carnivorous animals, which are considered to be the sylvatic reservoirs for visceral leishmaniasis.

The epidemiology of cutaneous leishmaniasis in the Americas is complex, with intra- and inter-specific variation in transmission cycles, reservoir hosts, sandfly vectors, clinical manifestations and response to therapy, and multiple circulating Leishmania species in the same geographical area. All the New World cutaneous leishmaniasis cycles are predominantly zoonotic, but the reservoir hosts vary by species and location, and in many cases are not fully known.

Both the geographic range and risk factors for cutaneous leishmaniasis transmission have expanded. In the past, New World cutaneous leishmaniasis was predominantly an occupational disease, related to activities such as rubber tapping, military operations, road construction and new agricultural development in forests and other enzootic areas. A classical example is the case of chiclero ulcer in southern Mexico, which has been described among workers who tap rubber trees. In forests, there are 'hot spots', i.e. well-delimited microfoci, where transmission is high as a result of favourable environmental conditions (e.g. climate, high densities of reservoirs and sandflies), whereas elsewhere in the forest the risk may be very low or absent. Occupational exposure remains important, but widespread deforestation has led to a rapid increase in cases and to peridomestic, periurban and even urban transmission. For example, between 1980 and 2001, there was a 10-fold increase in the incidence of cutaneous leishmaniasis and spread to all the states of Brazil. Population migration has also resulted in outbreaks in newly arrived immigrants entering cutaneous leishmaniasis-endemic zones, for example, in lowland Bolivia. 


\subsubsection{Socioeconomic factors}

Poverty increases the risk for leishmaniasis in many ways. Poor housing and peridomestic sanitary conditions (e.g. lack of waste management, open sewerage) may increase sandfly breeding and resting sites, as well as their access to humans. Crowding of a large number of people into a small space may attract peridomestic anthropophilic sandflies by providing a large biomass for blood-meals. Some transmission cycles, such as that of zoonotic visceral leishmaniasis in Brazil, are now concentrated in marginal neighbourhoods in periurban areas, where the sylvatic cycle may approach human dwellings. Economically driven migration may result in nonimmune individuals entering areas with transmission. Poor nutritional status resulting from a poor diet increases the likelihood that visceral leishmaniasis infection will progress to clinically manifest disease.

\subsubsection{Malnutrition}

Poor protein-energy, iron, vitamin A and zinc nutritional status increase the risk that an infection will progress to clinically manifest visceral leishmaniasis. Recent experiments in protein energy-, zinc- and iron-deficient mice suggest that this effect is mediated primarily through functional failure of the lymph node barrier and increased early visceralization of the parasite. Protein-energy malnutrition has also been associated with an increased risk for mucocutaneous leishmaniasis.

\subsubsection{Population movements}

Epidemics of both visceral and cutaneous leishmaniasis in both the Old and the New World are often associated with migration and the introduction of nonimmune people into areas with existing endemic or enzootic transmission cycles. Prediction of such outbreaks depends on the availability of ecological information and on evaluation of development areas before implementation of projects or population movements.

In the Andean countries, the lack of agricultural land in the highlands has resulted in massive migration of nonimmune populations to farm in low tropical forests in or near the Amazonian Basin, where cutaneous leishmaniasis is endemic. In some new settlement areas in Bolivia, $25 \%$ of the population showed active or healed lesions of cutaneous leishmaniasis within 3 years. Similarly, young people involved in agricultural development in Algeria and Saudi Arabia and in the construction of highways and railways in the Sahara have contracted L. major infection.

Military activities and conflict can also increase the incidence of the disease. In Colombia, military operations in the forest resulted in more than 45000 
cases of cutaneous leishmaniasis in soldiers between 2005 and 2010. Thousands of cases occurred in soldiers from the United Kingdom and the USA serving in Iraq and Afghanistan. Civilians are also affected: the civil war in southern Sudan forced people to move into zoonotic foci of visceral leishmaniasis in the western Upper Nile, culminating in the tragic kala-azar epidemic that claimed the lives of 100000 people.

Drought and harsh economic conditions can also force people to move into endemic regions; for example, a large number of cases of visceral leishmaniasis occurred in people who migrated from Darfur to Gedaref State in the Sudan. About $40 \%$ of all cases in India and Nepal occur in the districts bordering the two countries. Cross-border movement contributes to the spread of the disease and is a hindrance to the subcontinental visceral leishmaniasis elimination programme. The migration of numerous Afghan refugees into southern Pakistan introduced cutaneous leishmaniasis into areas where it had not previously been known.

\subsubsection{Environmental changes}

In most endemic regions, leishmaniasis is characterized by a patchy distribution with discrete transmission foci. This focal distribution of leishmaniasis transmission sites is due to microecological conditions that affect the vector, the parasite and the reservoir host. The transmission rate decreases rapidly as the distance from the transmission focus increases, because of the limited dispersal range of sandflies. Depending on the ecoepidemiology of a particular focus, natural changes or man-made modifications of these conditions can result in either an increase or a decrease in the incidence of the disease.

Environmental changes that can affect the incidence of leishmaniasis include urbanization, domestication of the transmission cycle and the incursion of agricultural farms and settlements into forested areas. The infection rate in zoonotic cutaneous and visceral leishmaniasis is often highest among people living at the edge of natural foci (e.g. forest, deserts), close to the sylvatic cycle. Latin American countries, particularly Brazil, have experienced large epidemics of zoonotic visceral leishmaniasis in the fast-growing peripheries of many large and medium-sized cities.

In the Old World, increased incidences of cutaneous leishmaniasis have been reported when suburbs extend into formerly uninhabited lands with a high density of rodent reservoir hosts. In some foci of anthroponotic cutaneous or visceral leishmanaisis, rural-to-urban migration accompanied by more poorquality suburban housing can increase the incidence of the disease.

In some epidemiological situations, deforestation and destruction of natural habitats can reduce transmission of leishmaniasis; however, in some cases, 
deforestation appears to have increased rather than decreased human infection. In former Soviet Central Asia, destruction of the burrows and vegetation habitats of $R$. opimus played a major role in the control of zoonotic cutaneous leishmaniasis. Similarly, in the Gedaref and Blue Nile states of the Sudan, introduction of mechanized agriculture and clearing of Acacia seyal and Balanites aegyptiaca woodland resulted in a substantial reduction in visceral leishmaniasis cases in the 1970s and early 1980s. The regrowth of Acacia seyal forests in the Upper Nile Province of southern Sudan was thought to be the main underlying factor in the visceral leishmaniasis epidemic that occurred in the late 1980s. More recently, large outbreaks of visceral leishmaniasis have occurred among migrants from Darfur who settled close to wooded areas along the Rahad and Atbara rivers in Gedaref State. In Colombia and Panama, forest-free zones created for cattle-breeding resulted in a buffer zone between the sylvatic cycle and human housing and reduced the transmission rate of cutaneous leishmaniasis caused by L. panamensis. In other parts of South America, however, deforestation appears to have led to an increase in the incidence of cutaneous leishmaniasis through a shift to a peridomestic transmission cycle.

\subsubsection{Climate change}

Leishmaniasis is a climate-sensitive disease, occupying a characteristic 'climate space' that is strongly affected by changes in rainfall, atmospheric temperature and humidity. Global warming and land degradation together are expected to affect the epidemiology of leishmaniasis by a number of mechanisms. First, changes in temperature, rainfall and humidity can have strong effects on the ecology of vectors and reservoir hosts by altering their distribution and influencing their survival and population sizes. Secondly, small changes in temperature can have a profound effect on the developmental cycle of Leishmania promastigotes in sandflies, potentially allowing transmission of the parasite in areas not previously endemic for the disease. Thirdly, drought, famine and flood resulting from changes in climate conditions could lead to massive displacement and migration of people to areas with transmission of leishmaniasis, and poor nutrition could compromise their immunity.

Although the close relation among climate conditions, sandfly seasonality and leishmaniasis is well documented, few studies have attempted to link interannual fluctuations in the incidence of leishmaniasis to climate cycles. Apart from predictive studies on possible expansion of leishmaniasisendemic zones to central Europe, no work has addressed the effects of longterm climate change on the disease. Epidemic waves of visceral leishmaniasis in northeastern Brazil were associated with human migration to urban areas after prolonged droughts. More recently, it was shown that the interannual 
fluctuations in leishmaniasis in Bahia (Brazil), Costa Rica and Colombia are associated with El Niño southern oscillation indices. Furthermore, a study in the Sudan and Tunisia showed that the annual incidence of visceral leishmaniasis over a certain period was correlated with annual rainfall in previous years.

Long-term climate change is predicted to expand the range of leishmaniasis and its sandfly vectors to regions that are currently free of both. The recent northwards spread of leishmaniasis in Italy was associated with a 30-year expansion of its vectors towards northern latitudes. A climatological analysis showed that an increase in temperature of $1{ }^{\circ} \mathrm{C}$ in July could lead to suitable conditions for the occurrence of $P$. neglectus spp. in certain parts of Austria, where they are not currently found. The observation of some Phlebotomus sandfly species in Germany and Belgium might be due to climate change and global warming, although it is also possible that these species migrated from the Mediterranean parts of Europe during the Holocene Climatic Optimum.

More studies are needed of the effects of climate factors on the epidemiology of leishmaniasis and to identify clear climate-related patterns of interannual fluctuations in the populations of vectors and reservoir hosts and the incidence of leishmaniasis. Such studies should include experimental and field investigations on the influence of climate variables on sandfly and Leishmania biology and spatial and temporal variations in leishmaniasis. The findings from such studies could help in forecasting future outbreaks, rationalizing health resources and achieving effective preparedness. Before they become useful for health planning, however, the validity of climate-leishmaniasis models should be determined with robust 'out-of-fit' data.

\subsubsection{Periodic fluctuations in incidence of disease}

Marked seasonal and interannual fluctuations in incidence are major characteristics of the epidemiology of leishmaniasis in many settings. Fluctuations are driven by climate factors, population dynamics of the vector and reservoir host, and human behaviour and movements. The predominant source of fluctuation in leishmaniasis transmission is the annual cycle of sandflies. Although sandflies are present throughout the year in tropical climates, each species tends to have its own annual cycle. The peak infection rate of Leishmania in vectors usually occurs when the maximum numbers of parous flies are present. The peak transmission rate is the product of vector abundance and vector infection rate; the degree of human-fly contact (which may also vary seasonally, e.g. if sleeping behaviour is seasonal) also plays a role.

These annual cycles are important for the prediction of transmission seasons and for the design and timing of control tactics. In addition, because the incubation period varies widely (from less than 1 month to more than 2 years), 
human incidence may not be clearly linked to the seasonal transmission rate. Fluctuations over longer periods have also been reported. Hypotheses to explain these cycles include accumulation of susceptible individuals in the population, resulting in lower herd immunity; the presence of infectious individuals (with PKDL playing a particularly important role in damping periodicity in theoretical models); amount of rainfall and occurrence of droughts. Concepts of cyclic periodicity in the endemicity of leishmaniasis require more in-depth research and empirical validation before they can be applied to the prediction of outbreaks.

\subsubsection{Epidemiological research and mathematical models}

Over the past two decades, much information on leishmaniasis has been obtained with quantitative epidemiological methods, including identification of risk factors (reviewed in section 2.6.1) and control measures, such as vector control, use of a buffer area around human dwellings for sylvatic leishmaniasis and reservoir host control. A number of mathematical models have been developed to determine incidence or transmission rates from cross-sectional data. Knowledge of the biology of vector species assists in the construction of mathematical models of the transmission of leishmaniasis. Predictive models would make it possible to anticipate epidemics and monitor control activities. The missing information includes the life expectancy of infected female sandflies; gonotrophic concordance versus discordance; the natural period of ovipositional cycles; the frequency of feeding on humans and on reservoir hosts or animals that play no part in transmission; and the time from ingestion of an infecting blood-meal to an infective bite. Mathematical models of the transmission of anthroponotic forms of leismaniasis would be simpler to construct than models of the transmission of zoonotic forms, which are complicated by the need to consider animal reservoir hosts.

Trials of insecticide application in houses and animal sheds and of insecticidetreated materials have shown effects on sandfly density or human landing rates in Bangladesh, Brazil, Colombia, India, Nepal and the Sudan. There is evidence, however, that insecticide treatment of animal sheds can sometimes repel sandflies, resulting in higher biting rates of nearby unprotected humans; future evaluations should take this into account. Little information is available to relate sandfly density to infection rates; the only published data are derived from a mathematical model of the epidemiology of canine leishmaniasis. Clearly, further work is urgently required on the association between vector abundance and clinical disease.

Use of a cleared barrier around human dwellings was assessed as a control measure for sylvatic zoonotic cutaneous leishmaniasis in French Guiana. Human cutaneous leishmaniasis incidence, sandfly density and reservoir 
hosts all decreased substantially in the buffer zone; however, the only control was historical. Similar environmental control, involving clearing of vegetation around houses, has been used on a small scale in forests in Central and South America and in parts of western Asia, but few results have been reported in the literature.

Ideally, evaluations of vector control should be based on the effect on the incidence of human disease. The incidence of clinical leishmaniasis is generally relatively low, however, so that the intervention and control populations must be chosen carefully, the sample must be large, and substantial resources should be available. Published intervention trials with leishmanial disease as the outcome are therefore few. Significant protection from anthroponotic cutaneous leishmaniasis by the use of insecticide-treated nets and other materials has been observed in Afghanistan, the Islamic Republic of Iran and the Syrian Arab Republic. A retrospective analysis of mass distribution of insecticide-treated nets in the Sudan showed a significant protective effect. On the Indian subcontinent, indoor residual spraying in the 1950s and 1960s for malaria control was associated with a steep decline in the visceral leishmaniasis incidence; however, there is no published report of the effect.

Epidemiological models to predict disease incidence and the effect of interventions would be useful for programme development and evaluation. As yet, there is insufficient regular collection of data to allow the use of models in the planning and evaluation of control programmes.

\subsubsection{Geographical information systems}

Geographical information systems, computer-based spatial techniques used to input, store, retrieve, manipulate, analyse, integrate and output geographically referenced or spatial data, are becoming important tools for understanding the epidemiology of leishmaniasis. Such systems integrate a wide range of data from different sources, including remote sensing and global positioning systems. Furthermore, geographical information systems can be flexibly adapted to the needs of endemic countries and geographical regions. When used properly, these systems can facilitate decision-making and support strategic planning for resource allocation and effective leishmaniasis control. Combined with good data from surveillance activities, these systems can be used routinely to generate base maps, delineate the distribution of vectors and reservoir hosts and prepare maps based on leishmaniasis prevalence or incidence. Comparing such maps with previous ones shows changes in the distribution of vectors, reservoir hosts and the disease. After proper analysis of environmental factors obtained through remote sensing and ground-based surveys, geographical information system models can be used to produce risk maps that can predict the probability of the presence of vectors 
and reservoir hosts and the presence or incidence of leishmaniasis in locations not covered by ground-based surveys.

In addition, geographical information systems include essential functionalities that can generate important information. For example, the distance calculator can be used to determine distances from forests, rivers, hospitals and health centres. Similarly, the 'digital elevation model' component can be used to calculate deviations such as slope, aspect and wetness index, which can be used in many studies of vector and reservoir host ecology and leishmaniasis epidemiology.

Although not yet used to their full potential, geographical information systems have been used in a number of studies on leishmaniasis, including mapping of important species of sandflies such as $P$. orientalis and P. martini in Ethiopia, Kenya, Somalia and the Sudan and $P$. papatasi in South-East Asia. Similarly, they were used to map the incidence of visceral leishmaniasis in eastern Sudan and the northwestern Bahia area of Brazil and the incidence of cutaneous leishmaniasis in Colombia and Tunisia. Furthermore, systematic use of such systems and standardized case-report forms has improved the gathering of epidemiological data on Leishmania-HIV coinfection in southwestern Europe and allowed visualization, analysis and monitoring of the spatial distribution of these cases.

\section{Epidemiological surveys of visceral leishmaniasis}

Epidemiological surveys may be performed as part of an outbreak investigation, to monitor and evaluate the impact of control measures or to assess whether an area is endemic for visceral leishmaniasis. Such surveys are used to determine the proportions of past and current infection, asymptomatic and symptomatic infection (visceral leishmaniasis) and acquired immunity in a population. As infection is usually not evenly distributed in a population, sampling all people in an area or village or sampling close contacts of recent cases in order to detect micro-foci is recommended. Local results should be extrapolated to other areas only with caution.

For epidemiological surveys to be fully informative, they should include use of a test to diagnose visceral leishmaniasis, use of the leishmanin skin test to determine acquired immunity and one or more tests to detect antibodies in asymptomatic people. All individuals should also be checked for the presence of PKDL. Age, gender, place of origin and recent visits to endemic areas should be recorded. A history of visceral leishmaniasis should be obtained for each person. The proportion who have a negative leishmanin skin test and in whom no antibodies can be detected should be determined; these people have not yet been infected and are thus susceptible to disease. 


\section{Use of diagnostic tests in epidemiological surveys}

The leishmanin skin test (sometimes called the Montenegro test in Latin America) is an intradermal test of the delayed-type hypersensitivity response, in which a suspension of formalinized Leishmania promastigotes is used as antigen. A simple technique for measuring induration of skin-test reactions ${ }^{5}$ is to apply moderate pressure, and slowly draw a line with a ballpoint pen from a point about $1-2 \mathrm{~cm}$ from the margin of the skin reaction towards its centre. When resistance to further movement is first felt (indicating the margin of the reaction), the pen is lifted from the skin. The procedure is then repreated on the opposite side of the skin reaction. This technique gives a visual record of the margins of induration, the diameter of which can be determined by measuring the distance between the opposing lines. A positive leishmanin skin test ( $\geq 5 \mathrm{~mm}$ diameter) is assumed to be indicative of cell-mediated immunity. The response is negative during active visceral leishmaniasis and becomes positive after cure, generally after months to 1 year. A positive response can also be seen after asymptomatic infection. A positive response is generally thought to be lifelong, but it can revert to negative over time. In endemic areas, the positivity rate is higher in adults than in children and increases with age. This pattern develops over time and is not seen when leishmaniasis has recently been introduced in a nonimmune population.

Tests to detect asymptomatic infection include a direct agglutination test IFAT and ELISA, which detect antibodies in asymptomatic people with visceral leishmaniasis infection at low cut-off titres. These titres may, however, represent cross-reactions with other parasitological infections, reducing the specificity of the test. In several studies, immunoblotting with 14- and $16-\mathrm{kDa}$ antigen proteins was found to be more sensitive and specific than ELISA in asymptomatic people living in the Mediterranean endemic area and in the Sudan; therefore, this test may be of value for epidemiological surveys. A combination of a direct agglutination test, IFAT or ELISA with a method that increases specificity, such as western blot or PCR, might be most practical for detecting asymptomatic infection in epidemiological surveys.

5 Sokal JE. Measurement of delayed skin test responses. New England Journal of Medicine, 1975;293:501-2. 



\section{Control}

As described in section 2, leishmaniasis transmission is maintained in a complex biological system involving the human host, parasite, sandfly vector and in some situations an animal reservoir. Therefore, control is unlikely to be achieved by a single intervention. A combination of case management strategies, integrated vector control and, if relevant, animal reservoir control is required and should be tailored to each context.

\subsection{Diagnosis}

\subsubsection{Visceral leishmaniasis}

\section{Clinical diagnosis}

The predominant complaints and clinical signs of visceral leishmaniasis are described in section 2.1 and Annex 3. These signs and symptoms, alone or in combination, are not specific enough to differentiate the condition from chronic malaria, schistosomiasis or other systemic infections. Visceral leishmaniasis should be suspected in a patient with fever and splenomegaly who lives in or has returned from an endemic area. In patients with HIV coinfection, the clinical features may be atypical (see section 2.1.11). The isolated or combined presence of anaemia, leukopenia, thrombocytopenia or polyclonal hypergammaglobulinaemia reinforces the clinical suspicion but lacks diagnostic accuracy. Therefore, Leishmania-specific laboratory tests are required for diagnostic confirmation.

\section{Parasitological diagnosis}

Visualization of the amastigote form of the parasite by microscopic examination of tissue aspirates is the classical confirmatory test for visceral leishmaniasis. Although the specificity of microscopy is high, its sensitivity varies, being higher for spleen (93-99\%) than for bone marrow (53-86\%) or lymph node (53-65\%) aspirates. Spleen aspiration can be complicated by lifethreatening haemorrhage in about $0.1 \%$ of individuals and therefore requires strict precautions, training and technical expertise, as well as facilities for nursing surveillance, blood transfusion and surgery (see Annex 4). Moreover, 
the accuracy of microscopic examination is influenced by the expertise of the laboratory technician and the quality of the reagents. Culturing of blood or organ aspirates increases the sensitivity of diagnosis.

The detection of parasite DNA by PCR in blood or bone marrow aspirates is substantially more sensitive than microscopic examination, although its use is currently restricted to referral hospitals and research centres. Easily readable formats of PCR that do not require sophisticated equipment are being developed to extend application. Positive PCR results can be found in few patients with febrile splenomegaly who do not suffer from visceral leishmaniasis but from other diseases. Because of its high sensitivity, PCR detects more asymptomatic infections than microscopic examination. Quantitative PCR techniques may in future prove more specific for acute disease, but proper standardization of PCR techniques and evaluation of their diagnostic accuracy in representative clinical settings are needed.

\section{Serological diagnosis}

Serological tests based on IFAT, ELISA or western blotting have shown good diagnostic accuracy in most studies but require equipment that is poorly adapted to field settings. The formol gel (or aldehyde) test is obsolete and should no longer be used for diagnosis. Two serological tests - the direct agglutination test and the rK39 antigen-based immunochromatographic test - were specifically developed for field use and have shown good diagnostic accuracy in most endemic areas. The rK39-based tests are easy to perform, quick, cheap and give reproducible results and can therefore be used for early diagnosis of visceral leishmaniasis at both peripheral and central levels (Annex 5). It improve the access of patients with visceral leishmaniasis to care in poor rural areas where most of them live.

All serological tests suffer from two limitations: first, specific antibodies remain detectable up to several years after cure. Therefore, relapse cannot reliably be diagnosed by serology; secondly, a significant proportion of healthy people living in endemic areas with no history of visceral leishmaniasis are positive for antileishmanial antibodies owing to asymptomatic infections. Antibody-based tests must therefore always be used in combination with a standardized clinical case definition for visceral leishmaniasis diagnosis (see Annex 3). As a number of counterfeit immunochromatographic tests have already been observed on the Indian subcontinent, the need for quality standards and regulation of diagnostics should be addressed (see section 3.7.3).

\section{Antigen-detection tests}

In theory, antigen-detection tests should be more specific than antibodydetection tests, as they avoid cross-reactivity and can distinguish active from 
past infections. A latex agglutination test to detect a heat-stable, low-relativemolecular-mass carbohydrate antigen in the urine of patients with visceral leishmaniasis showed good specificity but low-to-moderate sensitivity in East Africa and on the Indian subcontinent. Further work is needed to improve its sensitivity, reproducibility and feasibility.

\section{Diagnostic policy for health services in endemic areas}

A diagnostic policy should be drawn up that is specific for the health service, as the available laboratory techniques depend strongly on the level of the health system (see Table 3). In first-line centres and rural district hospitals in highly endemic areas, the rK39 antigen-based immunochromatographic test should be used (see Annex 5). Clinically suspect patients with a positive rK39 test and no history of visceral leishmaniasis should be treated. In areas where the sensitivity of the rK39 test is below $90 \%$, an additional serological (e.g. direct agglutination test) or parasitological test may be necessary if the results of the rK39 test are negative. Parasitological diagnosis, which is also necessary to diagnose a relapse of visceral leishmaniasis, should be performed at district or higher health facility level. In areas of low endemicity, such as the Mediterranean region, more specific diagnostic algorithms are required that would include PCR and parasitology in blood and bone marrow.

Table 3.

Minimal platform of techniques for diagnosis of visceral leishmaniasis in highly endemic areas, by health system level

\begin{tabular}{ll}
\hline Level of health system & Diagnostic tests \\
Primary health care centre & rK39 antigen-based immunochromatographic test \\
District hospital & $\begin{array}{l}\text { rK39 antigen-based immunochromatographic test, direct } \\
\text { agglutination test }\end{array}$ \\
& $\begin{array}{l}\text { Microscopy on bone marrow, spleen or lymph node } \\
\text { aspiration }\end{array}$ \\
Tertiary care (referral hospital) & $\begin{array}{l}\text { Serology: rK39 antigen-based immunochromatographic } \\
\text { test, direct agglutination test, other serological test (e.g. }\end{array}$ \\
& $\begin{array}{l}\text { IFAT, ELISA) } \\
\text { Microscopy on samples of buffy coat, spleen, bone } \\
\text { marrow, lymph nodes } \\
\text { Culture or PCR }\end{array}$
\end{tabular}

\footnotetext{
a Direct agglutination test should be used only if proper supervision and quality assurance are available at district level.

${ }^{b}$ Spleen aspiration should be performed only by experienced medical personnel and if facilities to treat bleeding complications are available.
} 


\subsubsection{Cutaneous leishmaniasis}

The clinical spectrum of cutaneous leishmaniasis is broad and may mimic that of other skin conditions, such as staphylococcal or streptococcal infection, mycobacterial ulcer, leprosy, fungal infection, cancer, sarcoidosis and tropical ulcer. As the clinical presentation of cutaneous leishmaniasis lacks specificity and treatment is costly, cumbersome or toxic, diagnostic confirmation is required.

\section{Parasitological diagnosis}

Parasitological diagnosis remains the reference standard in diagnosis of cutaneous leishmaniasis because of its high specificity. The sensitivity varies over a broad range, however, depending on geographical location, species and stage of the lesion. Multiple parasitological diagnostic tests should therefore be perfomed on each patient. Material for parasitological diagnosis can be obtained by scraping, fine- needle aspiration or biopsy of lesions (see Annex 2). A 2- to 4-mm skin punch biopsy provides more abundant material (an advantage when parasites are scarce) and allows culturing or histopathology for alternative diagnoses (e.g. for mycobacteria and fungi). Moreover, impression smears from the biopsy can be stained and examined.

The material obtained by any of these methods can be used for microscopic examination, culture and molecular diagnostic techniques. Culture from a biopsy sample must be homogenized in saline or culture medium under sterile conditions, which adds complexity to the procedure. Microscopic examination of Giemsa-stained material is often the only available method at primary, secondary or tertiary health care level in endemic areas. Culture of the parasite on simple media (see Annex 2) allows species identification and characterization (see section 2.3.3). Detection of parasite nucleic acids by molecular diagnosis, essentially by PCR-based methods, improves the diagnostic sensitivity and allows identification of the Leishmania species. This is particularly useful in regions (e.g. the New World) where several Leishmania species with various clinical outcomes and responses to treatment coexist. Multiplex PCR assays allow rapid species identification and are used increasingly, especially for New World cutaneous leishmaniasis. Both culture and molecularbased diagnosis require substantial laboratory infrastructure and technical expertise, limiting their use to reference laboratories.

\section{Immunological diagnosis}

Serological diagnosis is of limited use in cutaneous leishmaniasis because of its low sensitivity and variable specificity. The leishmanin skin test may be useful in epidemiological studies but is of little value in the diagnosis of 
cutaneous leishmaniasis. Neither serological tests nor the leishmanin skin test distinguishes between past and present infections.

\subsubsection{Mucocutaneous leishmaniasis}

Mucocutaneous leishmaniasis is caused by L. braziliensis, L. panamensis and, rarely, other species. Other diseases, such as allergic rhinitis, paracoccidioidomycosis and other deep mycoses, cancrum oris, lymphoma and other neoplasia, leprosy and sarcoidosis, may mimic early or advanced mucocutaneous leishmaniasis. A diagnosis can be strongly suspected in patients with typical mucosal lesions (see section 2.1.7) and a history of cutaneous leishmaniasis with one or more visible scars or, more rarely, with concomitant cutaneous leishmaniasis. Positive serology (e.g. IFAT, ELISA) or a positive leishmanin skin test increases clinical suspicion. A rise in antibody titres may be an early indicator of relapse. Parasites are scarce in mucosal lesions because of the strong local immune reaction; therefore, a search for parasites in mucosal samples (obtained by brushing or biopsy) by microscopic examination or by culture lacks sensitivity. The demonstration of parasite DNA by PCR has proved to be the most sensitive approach to confirm mucosal leishmaniasis.

\subsubsection{Post-kala-azar dermal leishmaniasis}

As the vast majority of cases of PKDL occur in patients with previous or concomitant visceral leishmaniasis in endemic rural areas of South Asia or East Africa, the diagnosis is mainly clinical. The diagnosis of PKDL can be confirmed by finding parasites in skin lesion samples obtained by biopsy or scraping of skin slit (see Annex 2). Studies in India have shown that smears are more likely to show amastigotes if they are taken from nodular lesions rather than papular or macular lesions. Skin biopsies can be examined by histopathology, immunohistochemistry and culture. PCR can be performed on skin biopsy samples or slit skin specimens in well-equipped laboratories and is substantially more sensitive. Serological tests such as the direct agglutination test, ELISA and the rK39 rapid diagnostic test are usually positive but are of limited value, as a positive result may be due to antibodies persisting after a past episode of visceral leishmaniasis. Nevertheless, serology can be helpful when other diseases (e.g. leprosy) are considered in the differential diagnosis or when a previous history of visceral leishmaniasis is uncertain.

\subsubsection{Coinfection with Leishmania and HIV}

Where HIV counselling and access to antiretroviral treatment are available, all visceral leishmaniasis patients should be screened for HIV, as the treatment options and prognosis differ in HIV-coinfected patients, who show 
decreased cellular and humoral responses to Leishmania spp. While the clinical manifestations in HIV-infected visceral leishmaniasis patients without severe immunosuppression are similar to those in immunocompetent patients, atypical clinical features can be found in patients with a low CD4+ lymphocyte count $(<200$ cells $/ \mu 1)$. In the latter group, physicians should suspect visceral leishmaniasis even in the absence of classical signs, such as splenomegaly. A substantial proportion of HIV-visceral leishmaniasis patients may present with other opportunistic infections, which complicate the clinical diagnosis. The parasite load is higher, and parasites may be found at unusual sites, especially in severely immunosuppressed patients. Therefore, microscopic examination, culture or PCR of blood (plain blood or buffy coat) or bone marrow aspirates is more sensitive than in immunocompetent patients. Bone-marrow aspirates from HIV-coinfected patients may, however, be paucicellular and therefore contain few parasites. Leishmania parasites can occasionally be found in biopsies from the skin, gastrointestinal tract or lungs.

The latex agglutination test has shown high sensitivity in detecting antigen in the urine of HIV-coinfected visceral leishmaniasis patients. In contrast, the sensitivity of serological tests is decreased in coinfected patients, and the study results are equivocal due to several factors, such as test format, region of endemicity and level of immunosuppression. Sensitivity can be increased by use of a sequential combination of different serological tests, e.g. the rK39 rapid diagnostic test and the direct agglutination test. In areas with a high prevalence of coinfection (e.g. Ethiopia), all visceral leishmaniasis patients should be tested for HIV infection.

HIV-coinfected patients also have a higher risk for atypical or more severe presentations of cutaneous and mucosal leishmaniasis. In endemic areas, patients with unusual presentations should be tested for HIV infection.

\subsection{Treatment and vaccines}

\subsubsection{General considerations}

Treatment should be given only after confirmation of disease (see section 3.1 and Annex 3). At the same time, the extent of concomitant infection should be ascertained, as this may influence the choice of therapy or supportive treatment. In some regions, the diagnosis should be to species level (e.g. New World cutaneous leishmaniasis). The treatment regimen should follow national and regional guidelines, if applicable. In many cases, supportive treatment, for example rehydration or nutritional supplementation, may be required before the start of therapy. Treatment should be given under the supervision of medical personnel. 
The drug policy in endemic countries and therapeutic decisions should be based on the individual benefit-risk ratio of medicines, the health service setting, the availability of antileishmanial medicines and public health considerations, such as the prevention of drug resistance. A country policy based on drug combinations would appear to be the best strategy for protecting medicines from resistance, if the available clinical evidence is confirmed in larger phase- 4 trials.

\subsubsection{Antileishmanial medicines}

\section{Pentavalent antimonials}

Two pentavalent antimonials are available: meglumine antimoniate and sodium stibogluconate. They are chemically similar, and their toxicity and efficacy are related to their antimonial content: meglumine antimoniate solution contains $8.1 \% \mathrm{Sb}^{5+}(81 \mathrm{mg} / \mathrm{ml})$, whereas sodium stibogluconate solution contains $10 \% \mathrm{Sb}^{5+}(100 \mathrm{mg} / \mathrm{ml})$. The injection may be given intramuscularly or intravenously either by infusion (over 5-10 $\mathrm{min}$ ) or by slow injection through a fine needle (23-25 gauge; $0.6-0.5 \mathrm{~mm})$ to avoid any risk of subsequent thrombosis. Pentavalent antimonials can be administered intralesionally for the treatment of cutaneous leishmaniasis.

Common side-effects are anorexia, vomiting, nausea, abdominal pain, malaise, myalgia, arthralgia, headache, metallic taste and lethargy. Electrocardiographic changes depend on the dose and duration of treatment, the commonest being T- wave inversion, a prolonged Q-T interval and arrhythmia. Cardiotoxicity and sudden death are serious but uncommon side-effects. Prolongation of a corrected Q-T interval $(>0.5 \mathrm{sec})$ signals the likely onset of serious and fatal cardiac arrhythmia. Pancreatic enzyme concentrations are commonly raised, but clinical pancreatitis is uncommon. Elevated liver enzyme concentrations, leukopenia, anaemia and thrombopenia are not uncommon. Patients should be monitored by serum chemistry, complete blood counts and electrocardiography. The quality of the pentavalent antimonials, like other medicines, should be assured (see section 3.7.3), as substandard medicines can cause severe toxicity and death. If serious side-effects arise (in most cases hepato- or cardiotoxicity), the drug should be changed.

\section{Amphotericin B deoxycholate}

Amphotericin B is a polyene antibiotic. A test dose of $1 \mathrm{mg}$, given by infusion, is recommended, followed by a full dose $4-6 \mathrm{~h}$ later. Infusion reactions, including higher fever, rigor and chills, are common, as is thrombophlebitis of the injected vein. Nephrotoxicity is also common, leading to frequent interruption of treatment in some patients. Other uncommon but serious toxic effects are hypokalaemia and myocarditis. Proper hydration and potassium 
supplementation are important. Treatment should always be given in hospital to allow continuous monitoring of patients.

\section{Lipid formulations of amphotericin B}

Several formulations, including liposomal amphotericin B, amphotericin B lipid complex and amphotericin B colloidal dispersion, have been used in treatment. They are similar to amphotericin B deoxycholate in their efficacy but are significantly less toxic. They are given by intravenous infusion over $2 \mathrm{~h}$. Mild infusion reactions (fever, chills and rigor) and back pain occur in some patients. Transient nephrotoxicity or thrombocytopenia is occasionally seen. Most clinical trials have been conducted with a reference liposomal amphotericin B formulation; all other lipid formulations should be evaluated for toxicity, bioequivalence and efficacy before they are used clinically.

\section{Paromomycin}

Paromomycin (aminosidine) is an aminoglycoside antibiotic, usually administered intramuscularly. The $15 \mathrm{mg} / \mathrm{kg}$ sulfate is equivalent to $11 \mathrm{mg} / \mathrm{kg}$ of base, and the $20 \mathrm{mg} / \mathrm{kg}$ sulfate is equivalent to $15 \mathrm{mg} / \mathrm{kg}$ of base. Mild pain at the injection site is the commonest adverse event (55\%). Reversible ototoxicity occurs in $2 \%$ of patients. Renal toxicity is rare. Some patients may develop hepatotoxicity, indicated by raised hepatic enzyme concentrations; tetany has also been reported. A topical formulation is available for cutaneous leishmaniasis.

\section{Pentamidine isethionate}

This drug is given intramuscularly or, preferably, by intravenous infusion. Severe adverse effects - diabetes mellitus, severe hypoglycaemia, shock, myocarditis and renal toxicity—-limit its use.

\section{Miltefosine}

This alkyl phospholipid (hexadecylphosphocholine) was originally developed as an oral anticancer drug but was shown to have antileishmanial activity. Miltefosine commonly induces gastrointestinal side-effects such as anorexia, nausea, vomiting (38\%) and diarrhoea (20\%). Most episodes are brief and resolve as treatment is continued. Occasionally, the side-effects can be severe and require interruption of treatment. Skin allergy, elevated hepatic transaminase concentrations and, rarely, renal insufficiency may be observed. Miltefosine should be taken after meals, and, if multiple doses are to be taken, they should be divided. Miltefosine is potentially teratogenic and should not be used by pregnant women or women with child-bearing potential for whom adequate contraception cannot be assured for the duration of treatment and for 3 months afterwards. 
Azoles medicines: ketoconazole, fluconazole, itraconazole

These oral antifungal agents have variable efficacy in leishmaniasis treatment (see section 3.2.3).

\subsubsection{Treatment options}

In this section, antileishmanial medicines and treatment regimens are qualified by grade of evidence (adapted from Cochrane reviews), as follows: (A) evidence obtained from at least one properly designed randomized controlled trial; (B) evidence obtained from well-designed trials without randomization; (C) opinions of respected authorities, based on clinical experience, descriptive studies or reports of expert committees; and (D) expert opinion without consistent or conclusive studies.

\section{Visceral leishmaniasis}

For the past seven decades, pentavalent antimonials have been the standard first- line medicines for visceral leishmaniasis. Amphotericin B deoxycholate and pentamidine have been used as second-line medicines. In the past 10 years, lipid formulations of amphotericin B, miltefosine and paromomycin have been approved for the treatment of visceral leishmaniasis; however, the efficacy and required dosage of several of these medicines have not been demonstrated in all endemic areas and may differ between these areas (see section 3.2.3).

Ideally, treatment for visceral leishmaniasis should cure the patient, reduce the risk for relapse and for PKDL and reduce transmission of resistant parasites. To ensure compliance and completion of the course of therapy, direct observation should be implemented, mainly for oral miltefosine. The treatment options for visceral leishmaniasis are summarized in Box 1.

Poor prognostic factors in antimony-treated visceral leishmaniasis patients are age $>45$ years (in Africa), malnutrition (hypoalbuminaemia, oedema), renal and hepatic comorbidity, concomitant infections, such as pneumonia, tuberculosis or HIV infection, or other immunosuppressive conditions. Supportive treatment is important; patients should be properly hydrated and given nutritional supplements. Severe anaemia should be corrected with blood transfusions, and concomitant infections should be treated with appropriate anti-infective agents. Successful therapy improves the general condition, resolves fever and causes regression of splenomegaly and recovery of blood counts towards normal. An initial cure can be declared if there is clinical improvement at the end of treatment. Complete regression of splenomegaly may take several months. A good indicator of definitive cure is the absence of clinical relapse at 6 months. 
Pentavalent antimonials: Sodium stibogluconate and meglumine antimoniate have been standard first-line medicines in most parts of the world $(>90 \%$ overall cure rate) (A), but drug resistance is a major concern in the Bihar focus, India, and in Nepal, where unresponsiveness is as high as $60 \%$, although this does not necessarily represent parasite resistance. Initial treatment of visceral leishmaniasis should be based on a daily injection of $20 \mathrm{mg} / \mathrm{kg}$ body weight of $\mathrm{Sb}^{5+}$ (no upper limit of $850 \mathrm{mg}$ ). Injections are usually given for 28-30 days.

Miltefosine at a dose of $2.5 \mathrm{mg} / \mathrm{kg}$ per day for 28 days to children aged 2-11 years and for people aged 12 years and above at a dose of $50 \mathrm{mg} /$ day for those weighting $<25 \mathrm{~kg}, 100 \mathrm{mg} /$ day for $25-50 \mathrm{~kg}$ body weight and $150 \mathrm{mg}$ /day for $>50 \mathrm{~kg}$ body weight for 28 days, has shown a cure rate in immunocompetents of $94 \%$ in India (A) and about $90 \%$ in Ethiopia (A).

Amphotericin B deoxycholate given daily or on alternate days by intravenous infusion in $5 \%$ dextrose for $4 \mathrm{~h}$ at a dose of $0.75-1.0 \mathrm{mg} / \mathrm{kg}$ per day for 15-20 doses was 99\% effective in India (A); data are lacking for other endemic regions.

Lipid formulations of amphotericin B: The total dose requirements for treatment of visceral leishmaniasis vary by region. In India and in Europe, all three formulations are effective. Liposomal amphotericin B is the most extensively used formulation in visceral leishmaniasis. In India (L. donovani), a total dose of $\geq 10 \mathrm{mg} / \mathrm{kg}$ results in a cure rate of $>95 \%$ (A). Given at a dose of $3-4 \mathrm{mg} / \mathrm{kg}$ per day to a total dose of $15-24 \mathrm{mg} / \mathrm{kg}$, administered in various regimens, this compound had $90-98 \%$ efficacy in southern Europe (B) (L. infantum) and is the reference treatment for visceral leishmaniasis in clinical practice in the Mediterranean region. There is limited experience in East Africa, where studies show lower $(<90 \%)$ cure rates at a total dose of $20 \mathrm{mg} / \mathrm{kg}(\mathrm{B})$, and where a total dose of $30 \mathrm{mg} / \mathrm{kg}$ given in $6-10$ doses is now in limited use. Shorter treatments are effective. In India, a 90\% cure rate was found with a single dose of $5 \mathrm{mg} / \mathrm{kg}$, and a $98 \%$ cure rate with a single dose of $10 \mathrm{mg} / \mathrm{kg}$ (A). In the Mediterranean area, a 96\% cure rate was achieved with a total dose of $20 \mathrm{mg} / \mathrm{kg}$ administered in two doses (B). In East Africa, studies are under way to determine the minimum effective total dose.

Paromomycin (aminosidine) has been shown to be effective in Indian visceral leishmaniasis: a dose of $15 \mathrm{mg} / \mathrm{kg}$ paromomycin sulfate (11 mg base) for 21 days gave a cure rate of $93-95 \%$ (A). The efficacy was $85 \%$ in East Africa at an increased dose of $20 \mathrm{mg} / \mathrm{kg}$ (15 mg base) per day for 21 days (A). There is no experience with this drug in L. infantum foci (Mediterranean, South America). 
Given the significant advantages of use of drug combinations and the risk of development of resistance to established and new medicines in anthroponotic foci, the use of monotherapy should be limited to liposomal amphotericin B where possible.

Combination treatment has the potential advantages of: shortening the duration of treatment, thereby increasing compliance; reducing the overall dose of medicines, thereby reducing their toxic effects and cost; and reducing the probability of selection of drug-resistant parasites, thereby prolonging the effective life of the available medicines. Several trials of combinations have been conducted, with favourable results. Combination of paromomycin and antimonials resulted in a higher cure rate in visceral leishmaniasis patients in Bihar than antimonials alone, to which lack of response was common.

In East Africa, the combination of sodium stibogluconate at $20 \mathrm{mg} / \mathrm{kg} \mathrm{Sb}^{5+}$ plus paromomycin given at $15 \mathrm{mg} / \mathrm{kg}$ (11 $\mathrm{mg}$ base) for 17 days showed an efficacy of $93 \%$ (A). The safety and efficacy of the combination treatment and sodium stibogluconate alone were similar. Furthermore, there were no differences in the efficacy of the combination treatment in Ethiopia, Kenya and the Sudan, suggesting that this regimen could be used across the region.

In several phase 3 studies in India, three separate combinations showed 98-99\% cure rates (A). The treatment included two sequential administrations, with either liposomal amphotericin B (5 mg/kg, single infusion) plus 7 days' miltefosine (dosage as above) or liposomal amphotericn B $(5 \mathrm{mg} / \mathrm{kg}$, single infusion) plus 10 days' paromomycin ( $11 \mathrm{mg} / \mathrm{kg}$ base), and co-adminstration of 10 days' miltefosine plus 10 days' paromomycin (dose as above). No safety issues were recorded.

\section{Post-kala-azar dermal leishmaniasis}

There is limited evidence on the treatment of PKDL, which depends on the severity of the condition in individual patients. In India, clinical experience with treatment for up to 4 months with intermittent amphotericin B deoxycholate (20 days on, 20 days off) (C) or 3 months of continous miltefosine (A) proved successful, disappearance of the lesion being taken as the criterion of cure. In East Africa, PKDL is not routinely treated, as the majority of cases $(85 \%)$ heal spontaneously within 1 year. Only patients with severe (grade 3 ) or disfiguring disease, those with lesions that have remained for $>6$ months, those with concomitant anterior uveitis and young children with oral lesions that interfere with feeding are treated, with either sodium stibogluconate $\left(20 \mathrm{mg} / \mathrm{kg} \mathrm{Sb}^{5+}\right.$ per day) for up to 2 months or a 20-day course of liposomal amphotericin B at $2.5 \mathrm{mg} / \mathrm{kg}$ per day. In the Sudan, the end-point is flattening of lesions and improvement of dyschromia. 
In a randomized controlled clinical trial in the Sudan of patients with persistent PKDL ( $>6$ months), which is difficult to cure with medicines alone, the cure rate with combined immunochemotherapy was significantly better than that with chemotherapy alone (final cure rates, $100 \%$ versus $40 \%$ ). The vaccine consisted of a mixture of killed L. major adsorbed onto alum plus bacillus Calmette-Guérin (BCG), given four times at weekly intervals; the drug was sodium stibogluconate, at $20 \mathrm{mg} / \mathrm{kg} \mathrm{Sb}{ }^{5+}$ for 40 days (see section 3.2.6).

\section{Cutaneous leishmaniasis}

Many different therapeutic interventions, including topical, systemic and nonpharmacological treatments, have been described. As few of the trials were appropriately designed and reported, the level of evidence is variable. It has been shown that the infecting species and geographical region affect the efficacy of treatments and therefore treatment recommendations. In anthroponotic cutaneous leishmaniasis there appears to be a higher risk for selection of drug- resistant parasites.

Cutaneous leishmaniasis is not a life-threatening condition, and severe complications are infrequent. As superficial secondary infections may complicate ulcerated cutaneous leishmaniasis, it is important to clean lesions. Mucosal involvement is exceptional in the Old World and is generally restricted to L. braziliensis and L. panamensis infections in the New World. Some species (especially L. major) are associated with a self-cure rate $>50 \%$ at 6 months. Usually, the recommended drug or treatment approach in cutaneous leishmaniasis should not induce life-threatening complications; however, in severe cases, the risk-benefit ratio is different.

The treatment decision is based firstly on the risk-benefit ratio of the intervention for each patient. Use of a treatment option with a risk for severe adverse events is acceptable if the patient suffers from numerous (typically, more than four), disfiguring face or complicated lesions, the size or location of which make local therapy impossible, or if local therapy has been tried and failed. Appropriate attention to contraindications and follow-up are important. In patients with mild disease or with comorbid conditions, a safer treatment should be preferred, even if the level of evidence for efficacy is weak.

Old World cutaneous leishmaniasis: The treatment options for Old World cutaneous leishmaniasis are summarized in Box 2.

Local wound care with careful follow-up are indicated for patients fulfilling the following criteria:

- confirmed or strongly suspected infection with L. major;

- fewer than four lesions requiring immediate treatment; 
- lesions $<5 \mathrm{~cm}$ in diameter;

- no potentially disfiguring or disabling lesion (face, joints, toes, fingers);

- no immunosuppression; and

- possibility for follow-up.

The patient's agreement to this option must be obtained after a full explanation of the clinical risks and the inconvenience of other options. If at least one criterion is absent, local therapy should be proposed. Local therapy is an attractive option with little toxicity, but intralesional treatment and, in much less degree thermotherapy, cause significant discomfort.

The options are:

Paromomycin ointments: A formulation of $15 \%$ plus methyl benzethonium chloride $12 \%$ ointment twice daily for up to 20 days has shown the same efficacy as that of intralesional $\mathrm{Sb}^{5+}(70 \%$ cure rate $)$ in the Islamic Republic of Iran, Israel and the Sudan (A). A formulation of $0.5 \%$ gentamicin and $15 \%$ paromomycin was more effective than placebo in L. major cutaneous leishmaniasis (B).

Thermotherapy: One or two applications of localized heat $\left(50{ }^{\circ} \mathrm{C}\right.$ for 30 s) was as effective as intralesional $\mathrm{Sb}^{5+}(70 \%$ cure rate $)$ in Afghanistan (L. tropica) (A) and more effective (70\% cure rate) than systemic $\mathrm{Sb}^{5+}$ in $L$. major cutaneous leishmaniasis (A). The device is expensive but works on a battery, a significant advantage for field use. Initial evolution of the disease after thermotherapy is complicated by second-degree burns. Local anaesthesia is necessary.

Intralesional pentavalent antimonials: A dose of $0.5-5 \mathrm{ml}$ is injected into the base and margins of the lesion to produce complete blanching. Daily, every other day or weekly infiltrations until healing of the lesion (one to eight infiltrations) were effective in Afghanistan and the Syrian Arab Republic (L. tropica) (A) and in South Asia (excluding India) and the Mediterranean Basin. In the Islamic Republic of Iran, weekly intralesional administration gave rise to resistant L. tropica.

Cryotherapy with liquid nitrogen $\left(-195{ }^{\circ} \mathrm{C}\right)$ applied once or twice to the lesion weekly up to 6 weeks was $>95 \%$ effective in Egypt, Israel and Jordan but less effective (77\%) than intralesional $\mathrm{Sb}^{5+}$ in Turkey (B). Liquid nitrogen application requires specific (usually expensive) devices and a skilled health-care provider. Cryotherapy is widely available at dermatology departments but generally not in the field, and the supply chain for liquid nitrogen requires heavy equipment.

Treatment of cutaneous leishmaniasis with systemic antimonials was widely recommended for decades, but studies published since 1991 have changed 
the estimated risk-benefit ratio, as no placebo-controlled study has shown the efficacy of systemic antimony in Old World cutaneous leishmaniasis. Systemic antimony should therefore be used only when information on efficacy and safety are available and for severe and complex lesions. Patients with pre-existing cardiac, hepatic, renal, pancreatic or haematological morbidity or advanced age are at high risk for toxicity-related mortality and should not be treated with systemic antimonials. In patients in whom systemic treatment is the only option (see above), the schedule of pentavalent antimonials generally used is $20 \mathrm{mg} / \mathrm{kg} \mathrm{Sb}^{5+}$ per day intramuscularly or intravenously for 10-20 days, although there is no evidence for the optimal duration. The efficacy of oral miltefosine for L. major was low in the Islamic Republic of Iran (B). Oral fluconazole was more effective than placebo in one study in Saudi Arabia (A).

In foci of L. major (the Islamic Republic of Iran and Saudi Arabia), the combination of cryotherapy and intralesional injections of antimony was more effective than each technique alone (A). Cryotherapy with liquid nitrogen must induce blanching of the lesion for $10 \mathrm{~s}$ and be rapidly followed by intralesional injection of undiluted pentavalent antimonial $(0.5-2 \mathrm{ml}$ per lesion). Injections are generally feasible in facial lesions, with the exception of those on the eyelids, lips, nose or ears. Local anaesthesia is not helpful. Pentavalent antimonials plus oral pentoxyfylline was more effective than pentavalent antimonials alone in the treatment of $L$. major lesions in the Islamic Republic of Iran (A).

New World cutaneous leishmaniasis: The treatment options for New World cutaneous leishmaniasis are summarized in Box 3. The New World form tends to be more severe and to last longer than that of the Old World; some patients infected by L. amazonensis, L. mexicana and L. panamensis develop diffuse disease. The therapeutic decision must be based first on the riskbenefit ratio of the intervention for each patient; no single treatment approach fits all possible clinical presentations. Local therapy was considered unsuitable for the treatment of New World cutaneous leishmaniasis caused by $L$. braziliensis or $L$. panamensis because of the potential risk of metastasis; however, as systemic treatment does not guarantee prevention of later mucocutaneous leishmaniasis, which is found in $<5 \%$ of the cases, local treatments should be explored. It is now considered acceptable to use topical therapy in selected cases of New World cutaneous leishmaniasis.

Because self-healing of New World cutaneous leishmaniasis is rare and evolution of disease is potentially severe, antileishmanial treatment is usually proposed.

Experience with local therapy for New World cutaneous leishmaniasis is limited. The criteria for using local treatment are similar to those for the Old 
World form of the disease. The options proposed are thermotherapy and paromomycin. One to three applications of localized heat $\left(50{ }^{\circ} \mathrm{C}\right.$ for $\left.30 \mathrm{~s}\right)$ were about $70 \%$ effective in Colombia and Guatemala 3 months after treatment (A). Paromomycin at $15 \%$ plus methyl benzethonium chloride $12 \%$ ointment twice daily for 20 days was $70-90 \%$ effective against cutaneous leishmaniasis caused by L. mexicana, L. panamensis and L. braziliensis in Ecuador and Guatemala (B).

The systemic treatment options are pentavalent antimonials, pentamidine, paromomycin sulfate, miltefosine and ketoconazole.

Pentavalent antimonials: The overall cure rate 3 months after treatment is about $77-90 \%$ when pentavalent antimonials are given at a dose of $20 \mathrm{mg} / \mathrm{kg} \mathrm{Sb}^{5+}$ per day for 20 days (A). The efficacy was greater against cutaneous leishmaniasis caused by L. braziliensis and L. panamensis than that caused by L. mexicana. Cutaneous leishmaniasis caused by a given species (e.g. L. guyanensis) may respond differently in different areas. The treatment is less effective in children under 5 years of age.

Pentamidine at a dose of $3-4 \mathrm{mg} / \mathrm{kg}$ on alternate days, for a total of three or four doses, was as effective as pentavalent antimonials for curing cutaneous leishmaniasis caused by L. panamensis (B) or $L$. guyanensis in Brazil, Colombia, French Guiana and Suriname (C). It is, however, less efficient than antimonials against disease caused by L. braziliensis (B).

Paromomycin sulfate was $<60 \%$ effective against New World cutaneous leishmaniasis in Belize and Colombia (A).

Miltefosine at a dose of $2 \mathrm{mg} / \mathrm{kg}$ per day for 28 days is effective against cutaneous leishmaniasis caused by L. panamensis ( $70-90 \%$ cure rate) but has only limited effect against that caused by L. braziliensis and L. mexicana $(<60 \%$ cure rate) (B). The response of $L$. braziliensis cutaneous leishmaniasis to miltefosine may vary depending on the area (33\% cure rate in Guatemala and $88 \%$ in Bolivia) (B).

Ketoconazole at $600 \mathrm{mg}$ daily for 28 days was $76-90 \%$ effective in L. panamensis and L. mexicana cutaneous leishmaniasis in Guatemala and Panama (B).

Combination therapy has been used in some studies. Topical administration of the immunomodulator imiquimod as adjunct therapy to pentavalent antimonials in Peru accelerated cure in comparison with antimonials alone in patients experiencing a relapse (A). Injected autoclaved $L$. mexicana and $L$. amazonesis promastigotes and BCG have been tested in combination with systemic antimonials in Venezuela, with some success. 
Rare and complex cutaneous leishmaniasis (recidivans, diffuse): Recidivans leishmaniasis is characterized by few parasites and common relapses. No studies are available on which to base firm recommendations for treatment. Pentavalent antimonials might be used at a standard dose for initial treatment. If there is no response, a combination of antimonials with oral allopurinol at a dose of $20 \mathrm{mg} / \mathrm{kg}$ for 30 days could be used (C). Immunotherapy is still considered to be experimental.

Diffuse cutaneous leishmaniasis is caused by L. aethiopica or L. amazonensis and is characterized by frequent relapses. In Ethiopia, treatment with a combination of pentavalent antimonials and parenteral paromomycin for 60 days was successful in three patients (C). Pentamidine at a dose of $3-4 \mathrm{mg} / \mathrm{kg}$ once a week (for up to 4 months) is an option, but diabetes is a concern (C). Whatever the regimen used, treatment should be prolonged for several weeks beyond clinical cure.

\section{Mucosal leishmaniasis}

New World mucocutaneous leishmaniasis: The treatment options for mucocutaneous leishmaniasis are summarized in Box 4. The treatment outcome depends on the location of the lesions. High cure rates are obtained when the lesions are limited to the nose and mouth; when the larynx, vocal cords and trachea are involved, however, the cure rates after therapy with systemic antimonials are low, and relapse and recurrence are frequent after clinical improvement and apparent cure. The scarcity of amastigotes and the difficulty of culture usually complicate parasitological monitoring.

Pentavalent antimonials: Cure rates after treatment with pentavalent antimonials range from $30 \%$ to $100 \%$, depending on the location of lesions and the geographical area (C). The current regimen is $20 \mathrm{mg} / \mathrm{kg}$ per day for 30 days.

Amphotericin B deoxycholate: A regimen of 20-45 doses of 0.7-1.0 $\mathrm{mg} / \mathrm{kg}$ was $80-90 \%$ effective (C) but requires intravenous infusion and strict monitoring of renal function.

Liposomal amphotericin B: Liposomal amphotericin B at a dose of $2-3 \mathrm{mg} / \mathrm{kg}$ for at least 20 days gave similar cure rates as and fewer adverse events than amphotericin B deoxycholate (C).

Pentamidine: There are limited data on use of pentamidine, but it can be considered as an alternative treatment (C).

Miltefosine: In Bolivia, the cure rate of mucocutaneous leishmaniasis (L. braziliensis) under miltefosine $(2.5-3.3 \mathrm{mg} / \mathrm{kg}$ per day for 4 weeks) was $83 \%$ in patients with mild disease and $58 \%$ in patients with more extensive disease. The cure rates did not improve when therapy was prolonged from 4 to 6 weeks (B). 
A combination of oral pentoxyfylline plus pentavalent antimonials for 30 days reduced the relapse rate and accelerated cure in comparison with pentavalent antimonials alone (A).

\subsubsection{Special situations}

\section{Pregnancy and lactation}

Little information is available on the treatment of visceral leishmaniasis in pregnancy. The threat of a fatal outcome of leishmaniasis for the mother, the fetus and the newborn is much greater than the risk for drug adverse effects. When untreated, spontaneous abortion, small-for-birth date and congenital leishmaniasis have been described. Local treatment should be the first option for cutaneous leishmaniasis. Pregnancy influences the clinical manifestations of New World cutaneous leishmaniasis, pregnant women having larger, less typical lesions.

Amphotericin B deoxycholate and lipid formulations are the best therapeutic options for visceral leishmaniasis. No abortions or vertical transmission have been reported in mothers treated with liposomal amphotericin B (C).

Pentavalent antimonials are less safe in pregnancy, as they can result in spontaneous abortion, preterm deliveries and hepatic encephalopathy in the mother and vertical transmission $(\mathrm{C})$.

Paromomycin: Ototoxicity in the fetus is the main concern. Insufficient data are available on the use of paromomycin in pregnant women.

Pentamidine is contraindicated during the first trimester of pregnancy.

Miltefosine is potentially embryotoxic and teratogenic and should not be used during pregnancy. Women of child-bearing age should be tested for pregnancy before treatment and use effective contraception for 3 months after treatment.

\section{Leishmania-HIV coinfection}

Visceral leishmaniasis is an AIDS-defining condition and a valid entry point for starting antiretroviral treatment, irrespective of CD4+ count. The baseline CD4+ count is lower in Leishmania-HIV coinfected patients, as visceral leishmaniasis itself causes a reduction in CD4+ cells. The impact of antiretroviral treatment on visceral leishmaniasis in coinfected patients observed in the Mediterranean area can be summarized as: a reduction of incidence by $50-65 \%$, higher survival rates, a reduction in relapse rate and possible immune reconstitution inflammatory syndrome. 
HIV and Leishmania infection reinforce each other. HIV patients are more likely to develop visceral leishmaniasis (due to reactivation of a dormant infection or clinical manifestation after primary infection). Patients characteristically have high disseminated parasite loads. Visceral leishmaniasis negatively affects the response to antiretroviral treatment and is difficult to cure in coinfected patients, especially those with CD4+ counts $<200$ cells/ $\mu 1$, who typically relapse.

Cutaneous leishmaniasis, characterized by multiple, polymorphic, relapsing lesions, is more difficult to treat and there is a higher rate of recurrence. Systemic treatment of cutaneous leishmaniasis is recommended.

The prognosis of coinfected patients is characterized by a high mortality rate during the first episode, increased antileishmanial drug toxicity (predominantly with antimonials), poor long-term clinical response, parasitological cure and a high relapse rate over a lifetime. The risk factors for relapse are: no antiretroviral treatment, low CD4+ cell count, previous visceral leishmaniasis episode, failure to achieve clinical or parasitological cure during the first episode and no secondary prophylaxis.

Few clinical studies have been conducted of the efficacy of treatment for HIV- visceral leishmaniasis coinfection outside the Mediterranean area. Antimonials are more toxic in HIV patients, necessitating careful monitoring for pancreatitis and cardiotoxicity. Amphotericin B deoxycholate or lipid formulations should be considered first and pentavalent antimonials only in areas of no significant resistance and when lipid formulations of amphotericin $\mathrm{B}$ are unavailable or unaffordable. Lipid formulations infused at a dose of $3-5 \mathrm{mg} / \mathrm{kg}$ daily or intermittently for 10 doses (days $1-5,10,17,24,31$ and 38) up to a total dose of $40 \mathrm{mg} / \mathrm{kg}$ are recommended (A). Experience with miltefosine is limited. In Ethiopia, miltefosine was found to be less effective but safer than antimonials in coinfected patients (B). Combination regimens should be tested in coinfected patients, as they may improve treatment efficacy and reduce toxicity.

Evidence for the efficacy of maintenance therapy is limited to patients coinfected with $L$. infantum in the Mediterranean area. An amphotericin B lipid complex (3-5 mg/kg per dose once) given every 3 weeks for 12 months as secondary prophylaxis was well tolerated: after 1 year, only $22 \%$ of patients had relapsed, in comparison with $50 \%$ of patients without secondary prophylaxis (A). Other options are liposomal amphotericin B (3-5 mg/kg per dose once every 3-4 weeks) (C); pentavalent antimonials $\left(20 \mathrm{mg} \mathrm{Sb}^{5+} / \mathrm{kg}\right.$ per dose once every 3-4 weeks) (C) and pentamidine (4 mg/kg per dose once [300 $\mathrm{mg}$ for an adult] every 3-4 weeks) (C). Once a patient's immune function has recovered with antiretroviral treatment and visceral leishmaniasis is qui- 
escent, prophylaxis could be suspended, provided that the CD4+ cell count is maintained at $>200$ cells $/ \mu 1$ for more than 6 months $(B)$.

\section{Other forms of immunosuppression}

Atypical and relapsing cutaneous and visceral leishmaniasis in non-HIV immunosuppressed patients have been described. Patients suffering from hypogammaglobulinaemia, some autoimmune diseases or some cancers or who have received an organ transplant, who are being treated with TNF- $\alpha$ antagonists, corticosteroids or methotrexate are at risk. It is recommended that patients living in or returning from areas endemic for leishmaniasis undergo serological screening before immunosuppressive treatment. Secondary prophylaxis is often required, which can be stopped when immunosuppression ends.

\section{Box 1. Recommended treatment regimens for visceral leishmaniasis, ranked by preference ${ }^{1}$}

Anthroponotic visceral leishmaniasis caused by L. donovani in Bangladesh, Bhutan, India and Nepal

1. Liposomal amphotericin B: $3-5 \mathrm{mg} / \mathrm{kg}$ per daily dose by infusion given over 3-5 days period up to a total dose of $15 \mathrm{mg} / \mathrm{kg}(\mathrm{A})$ by infusion or $10 \mathrm{mg} / \mathrm{kg}$ as a single dose by infusion $(\mathrm{A})$

2. Combinations (co-administered) $(A)$

- liposomal amphotericin B (5 mg/kg by infusion, single dose) plus miltefosine (daily for 7 days, as below)

- liposomal amphotericin B (5 mg/kg by infusion, single dose) plus paromomycin (daily for 10 days, as below)

- miltefosine plus paromomycin, both daily for 10 days, as below

3. Amphotericin B deoxycholate: $0.75-1.0 \mathrm{mg} / \mathrm{kg}$ per day by infusion, daily or on alternate days for 15-20 doses (A)

4. Miltefosine: for children aged 2-11 years, $2.5 \mathrm{mg} / \mathrm{kg}$ per day; for people aged $\geq 12$ years and $<25 \mathrm{~kg}$ body weight, $50 \mathrm{mg} /$ day; 25-50 kg body weight, $100 \mathrm{mg} /$ day; > $50 \mathrm{~kg}$ body weight, $150 \mathrm{mg} /$ day; orally for 28 days (A)

or

Paromomycin: $15 \mathrm{mg}$ (11 mg base) per kg body weight per day intramuscularly for 21 days $(A)$

5. Pentavalent antimonials: $20 \mathrm{mg} \mathrm{Sb}^{5+} / \mathrm{kg}$ per day intramuscularly or intravenously for 30 days in areas where they remain effective: Bangladesh, Nepal and the Indian states of Jharkhand, West Bengal and Uttar Pradesh (A) 
Rescue treatment in case of non-response: conventional amphotericin B deoxycholate infusions or liposomal amphotericin B at higher doses

Visceral leishmaniasis caused by L. donovani in East Africa (Ethiopia, Eritrea, Kenya, Somalia, Sudan and Uganda) and Yemen

1. Combination: pentavalent antimonials $\left(20 \mathrm{mg} \mathrm{Sb}^{5+} / \mathrm{kg}\right.$ per day intramuscularly or intravenously) plus paromomycin (15 mg [11 mg base] per kg body weight per day intramuscularly) for 17 days (A)

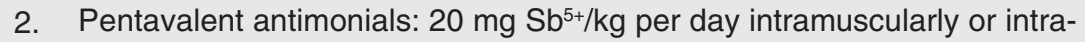
venously for 30 days $(A)$

3. Liposomal amphotericin $\mathrm{B}$ : $3-5 \mathrm{mg} / \mathrm{kg}$ per daily dose by infusion given over 6-10 days up to a total dose of $30 \mathrm{mg} / \mathrm{kg}(\mathrm{B})$

4. Amphotericin $B$ deoxycholate: $0.75-1 \mathrm{mg} / \mathrm{kg}$ per day by infusion, daily or on alternate days, for 15-20 doses (A)

5. Miltefosine orally for 28 days at dosage as above (A)

\section{Post-kala-azar dermal leishmaniasis}

\section{East Africa}

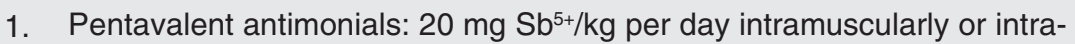
venously for 30-60 days, when indicated (C)

2. Liposomal amphotericin B: $2.5 \mathrm{mg} / \mathrm{kg}$ per day by infusion for 20 days, when indicated (C)

Bangladesh, India, Nepal

1. Amphotericin B deoxycholate: $1 \mathrm{mg} / \mathrm{kg}$ per day by infusion, up to $60-80$ doses over 4 months (C)

2. Miltefosine orally for 12 weeks at dosage as above $(A)$

Visceral leishmaniasis caused by L. infantum: Mediterranean Basin, Middle East, Central Asia, South America

1. Liposomal amphotericin $\mathrm{B}: 3-5 \mathrm{mg} / \mathrm{kg}$ per daily dose by infusion given over a 3-6 days period, up to a total dose of $18-21 \mathrm{mg} / \mathrm{kg}(\mathrm{B})$

2. Pentavalent antimonials: $20 \mathrm{mg} \mathrm{Sb}^{5+} / \mathrm{kg}$ per day intramuscularly or intravenously for 28 days $(B)$

3. Amphotericin B deoxycholate: $0.75-1.0 \mathrm{mg} / \mathrm{kg}$ per day by infusion, daily or on alternate days for 20-30 doses, for a total dose of 2-3 g (C)

1 See text for grades of evidence, details and precautions to be taken and for treatment options for HIV coinfections 


\section{Box 2. Recommended treatment regimens for Old World cutaneous leish- maniasis (not ranked by preference)}

No antileishmanial treatment (see text)

\section{Local therapy}

L. major

- $15 \%$ paromomycin $/ 12 \%$ methylbenzethonium chloride ointment twice daily for 20 days $(A)$

- Intralesional antimonials, $1-5 \mathrm{ml}$ per session plus cryotherapy (liquid nitrogen: $-195^{\circ} \mathrm{C}$ ), both every $3-7$ days ( $1-5$ sessions) (A)

- thermotherapy, $1-2$ sessions with localized heat $\left(50{ }^{\circ} \mathrm{C}\right.$ for $30 \mathrm{~s})(\mathrm{A})$

- intralesional antimonials or cryotherapy independently, as above (D)

L. tropica, L. aethiopica* and L. infantum*

- $15 \%$ paromomycin $/ 12 \%$ methylbenzethonium chloride ointment, as above (D)

- intralesional antimonials plus cryotherapy, as above (D)

- thermotherapy, as above (A)

- intralesional antimonials, alone, as above (B)

- cryotherapy, alone, as above (C)

\section{Systemic therapy}

L. major

- fluconazole, $200 \mathrm{mg}$ oral daily for 6 weeks $(\mathrm{A})$

- pentavalent antimonials, $20 \mathrm{mg} \mathrm{Sb}^{5+} / \mathrm{kg}$ per day intramuscularly or intravenously for 10-20 days (D)

- pentavalent antimonials, $20 \mathrm{mg} \mathrm{Sb}^{5+} / \mathrm{kg}$ per day intramuscularly or intravenously plus pentoxyfylline, $400 \mathrm{mg}$ three times a day for $10-20$ days (A)

L. tropica and L. infantum*

- pentavalent antimonials, $20 \mathrm{mg} \mathrm{Sb}^{5+} / \mathrm{kg}$ per day intramuscularly or intravenously for 10-20 days (D)

- pentavalent antimonials, $15-20 \mathrm{mg} \mathrm{Sb}^{5+} / \mathrm{kg}$ per day intramuscularly or intravenously for 15 days plus oral allopurinol 20 $\mathrm{mg} / \mathrm{kg}$ for 30 days, to treat leishmaniasis recidivans caused by L. tropica (C)

L. aethiopica

- pentavalent antimonials $20 \mathrm{mg} \mathrm{Sb}{ }^{5+} / \mathrm{kg}$ per day intramuscularly or intravenously plus paromomycin, $15 \mathrm{mg}$ (11 mg base)/ 
kg per day intramuscularly for 60 days or longer to treat diffuse cutaneous leishmaniasis (C)

* Few data are available on therapy for cutaneous leishmaniasis due to $L$. infantum and L. aethiopica.

\section{Box 3. Recommended treatment regimens for New World cutaneous leishmaniasis (not ranked by preference)}

\section{No anti-leishmanial treatment (see text)}

\section{Local therapy, all species}

- $15 \%$ paromomycin and $12 \%$ methylbenzethonium chloride ointment twice daily for 20 days (B)

- thermotherapy: $1-3$ sessions with localized heat $\left(50{ }^{\circ} \mathrm{C}\right.$ for $\left.30 \mathrm{~s}\right)$ (A)

- intralesional antimonials: $1-5 \mathrm{ml}$ per session every 3-7 days (1-5 infiltrations) (B)

\section{Systemic}

L. mexicana

- ketoconazole: adult dose, $600 \mathrm{mg}$ oral daily for 28 days (B)

- miltefosine: $2.5 \mathrm{mg} / \mathrm{kg}$ per day orally for 28 days (B)

L. guyanensis and L. panamensis

- pentamidine isethionate, intramuscular injections or brief infusions of $4 \mathrm{mg}$ salt $/ \mathrm{kg}$ per dose every other day for 3 doses $(C)^{*}$

- pentavalent antimonials: $20 \mathrm{mg} \mathrm{Sb}^{5+} / \mathrm{kg}$ per day intramuscularly or intravenously for 20 days $(\mathrm{C})^{*}$

- miltefosine: $2.5 \mathrm{mg} / \mathrm{kg}$ per day orally for 28 days $(B)$

L. braziliensis

- pentavalent antimonials: $20 \mathrm{mg} \mathrm{Sb}^{5+} / \mathrm{kg}$ per day intramuscularly or intravenously for 20 days (A)

- amphotericin B deoxycholate: $0.7 \mathrm{mg} / \mathrm{kg}$ per day, by infusion, for 25-30 doses (C)

- liposomal amphotericin B: 2-3 mg/kg per day, by infusion, up to $20-40 \mathrm{mg} / \mathrm{kg}$ total dose (C)

L. amazonensis, L. peruviana and $L$. venezuelensis

- pentavalent antimonials: $20 \mathrm{mg} \mathrm{Sb}^{5+} / \mathrm{kg}$ per day intramuscularly or intravenously for 20 days

Relapse treatment:

- amphotericin B deoxycholate, as above 
- pentavalent antimonials: as above plus topical imiquimod every other day for 20 days $(A)$

- liposomal amphotericin B: $3 \mathrm{mg} / \mathrm{kg}$ per day, by infusion, up to 20-40 mg/kg total dose may be considered

* The efficacy of pentamidine, and pentavalent antimonialsis depends on the geographical area (see text).

Box 4. Recommended treatment regimens for mucocutaneous leishmaniasis (not ranked by preference)

\section{All species*}

- pentavalent antimonials: $20 \mathrm{mg} / \mathrm{kg}$ per day intramuscularly or intravenously for 30 days $(\mathrm{C})$

- pentavalent antimonials: as above plus oral pentoxifylline at $400 \mathrm{mg} / 8 \mathrm{~h}$ for 30 days (A)

- amphotericin B deoxycholate: $0.7-1 \mathrm{mg} / \mathrm{kg}$ by infusion every other day up to $25-45$ doses (C)

- liposomal amphotericin B: 2-3 mg/kg daily by infusion up to a total dose of $40-60 \mathrm{mg} / \mathrm{kg}(\mathrm{C})$

- $\quad$ in Bolivia: miltefosine at $2.5-3.3 \mathrm{mg} / \mathrm{kg}$ per day orally for 28 days (B)

* Little data are available for the therapy of MCL due to L. aethiopica

\subsubsection{Prophylactic leishmaniasis vaccines}

There is no vaccine for general use against leishmaniases. As recovery from infection is usually accompanied by strong immunity and as it is possible to protect experimental animals against live challenge, hope for developing a vaccine for humans has been strong.

\section{Leishmanization}

Intradermal inoculation of live virulent $L$. major promastigotes from a fresh culture has been used intermittently for many years to protect against the more severe effects of natural $L$. major infection. The procedure may, however, induce severe cutaneous lesions and should not be used under normal conditions. Leishmanization with L. major is a useful live challenge for evaluating candidate vaccines for cutaneous leishmaniasis; however, it must be done under controlled conditions with high-risk volunteers without HIV infection. Trials must be conducted under the guidelines of good clinical practice, and inoculums must be produced in facilities certified for good manufacturing practice. 


\section{First-generation candidate vaccines}

The first-generation candidate vaccines against leishmaniasis have been made with whole killed parasites or extracts. Numerous attempts to develop such a vaccine have been made, in Brazil, Colombia, Ecuador, Venezuela and the Islamic Republic of Iran against cutaneous leishmaniasis and in the Sudan against visceral leishmaniasis. Three vaccine candidates have been tested: a L. amazonensis- based vaccine derived from an earlier five-valent vaccine in Brazil ('Mayrink vaccine') and later from a single parasite; L. mexicana produced in Venezuela and given with BCG ('Convit vaccine'); and an L. major vaccine produced in the Islamic Republic of Iran and given with BCG ('Razi Institute vaccine'). The results have been inconclusive or negative for prophylaxis but have been encouraging for therapeutic indications.

\section{Second-generation vaccines}

Second-generation vaccines consist of recombinant proteins and genetic vaccines. Many defined antigens can protect experimental animals when used with adjuvants, but so far only one defined vaccine, Leish-111f + MPL-SE, has reached clinical trials. It is being evaluated for the immunotherapy of PKDL in the Sudan, in phase- 1-2 trials in Peru and in a phase-1 trial in India.

Genetically modified Leishmania have been produced that cause little disease or infection. They have been shown to induce a protective response against live challenge in mice.

Recently, some financial support has been provided for vaccine development; however, it is unlikely that a prophylactic vaccine will be available within the next 5 years for any form of leishmaniasis.

\section{Canine leishmaniasis vaccines}

In Brazil, two canine vaccines have been commercialized. The results of phase-I and -2 studies of these vaccines showed a promising decrease in the severity of clinical disease among dogs, and the vaccines were licensed by the Ministry of Agriculture. The Ministry of Health does not, however, recommend their use for public health purposes, because there has been no conclusive study showing their potential effect in decreasing transmission rates. A key requirement for the use of such vaccines as a control measure for zoonotic visceral leishmaniasis is phase-3 studies of their effectiveness to prevent transmission. An additional operational problem is that vaccinated dogs cannot be distinguished from naturally infected dogs with the commonly used serological methods. The companies responsible for producing these vaccines had a deadline of 36 months from 9 July 2007 for conducting phase3 studies showing efficacy in decreasing transmission rates, in order to have their licences renewed. 
Protective immunity usually follows recovery from all forms of leishmaniasis in immunocompetent individuals, but the behaviour of leishmaniasis in these individuals suggests that the immunity is not sterile. Hence, the immune response plays an important role in the recovery of patients from leishmaniasis. The concept of stimulating the immune response during chemotherapy is not new and not specific for leishmaniasis. Immunotherapy with or without chemotherapy has been used for treatment of cutaneous leishmaniasis in Venezuela for more than a decade. Three injections of autoclaved L. mexicana mixed with BCG ('Convit vaccine') are given; if the patient does not respond, chemotherapy is initiated. The cost of the vaccine is much lower than that of chemotherapy. It appears to be safe and is not associated with the adverse effects of the antimonials, but it produces a lesion which may last for a few weeks. In Brazil, repeated daily doses of killed L. amazonensis vaccine ('Mayrink vaccine') are given with a low dose of antimonial $(8 \mathrm{mg} / \mathrm{kg}$ body weight per day) for four cycles of 10 daily injections, followed by a 10-day rest. The vaccine has been registered in Brazil as an adjunct for low-dose chemotherapy.

In the Sudan, a trial involving patients with persistent PKDL showed that the cure rate with immunochemotherapy was significantly higher than that with chemotherapy alone (final cure rates: $87 \%$ and $53 \%$ ). The vaccine was a mixture of killed L. major adsorbed on alum plus BCG, given four times at weekly intervals. The mechanism involved in enhancing the cure rate is not fully understood.

While there may be a therapeutic role for immunomodulators in the future, none is available or recommended for routine use at present.

As therapeutic vaccines can be evaluated rapidly at lower cost and proof of principle is already available, therapeutic (or immunochemotherapeutic) vaccines may be achievable.

\subsection{Detection}

\subsubsection{Passive case detection}

'Passive case detection' is detection triggered by patients seeking care for their illness from clinicians working in fixed health facilities. Clinicians who manage a case should notify it to the appropriate epidemiological surveillance system (see section 6.3). Use of standardized definitions for cases of visceral leishmaniasis, PKDL and cutaneous leishmaniasis is essential (see boxes 1-3 and Annex 3). Most countries endemic for visceral and cutaneous leishmaniasis rely on 'passive surveillance' for reporting their dis- 
ease burden. Such reporting, however, captures only a fraction of the true burden, as access to care for this neglected disease is limited and the cases managed in the private sector are usually not included in surveillance data. Passive case detection resulted in an underestimate of the incidence of visceral leishmaniasis in Bihar, India, at a ratio of 1:8, and cutaneous leishmaniasis was underestimated by a factor of 40 in Guatemala. Nevertheless, such data can be useful if interpreted with caution for documenting trends in the burden of visceral and cutaneous leishmaniasis in a country. To ensure sustainable monitoring, routine surveillance systems must be improved in the context of strengthening health care systems. For general information on routine surveillance systems, see section 6.3 .

\subsubsection{Active case detection}

Active case detection or search means that health staff reach out to the community and systematically screen the population to find cases of leishmaniasis. Active case search is an essential component of the visceral leishmaniasis elimination strategy on the Indian subcontinent. It should help to reduce disease transmission by shortening the infectious period of patients; and earlier diagnosis and treatment offer a clinical benefit. Preliminary data show that active case detection is cost-effective in areas where disease incidence is high, people's awareness about the disease is poor and health services (and thus passive case detection) are weak.

Several active case detection approaches are possible, such as:

- house-to-house search (or blanket screening). A medical team visits each house in a community and screens every member of the household.

- camp approach. A 'medical camp' is organized in an easily accessible place in a village, and the community is invited to attend the screening session. The camp should be preceded by a community awareness campaign.

- index case approach (or 'snowballing'). An 'index case' is a person who has recently been confirmed as having visceral leishmaniasis or PKDL. A house-to-house search in the immediate neighbourhood of the index case is conducted.

- incentive-based approach. An incentive or award is given to health volunteers who facilitate case detection.

Operational research has shown that the cost-effectiveness of each of these strategies depends on the local epidemiological context; blanket screening is the least efficient. Both the camp and the index case approach were found to be effective for detecting visceral leishmaniasis and PKDL, although the available diagnostic tools are not sufficiently sensitive or specific, making active case detection for PKDL frustrating. 
Active case detection has been a key measure in reducing the incidence of cutaneous leishmaniasis under epidemic conditions (e.g. in Bam, Islamic Republic of Iran) and has been used for detecting visceral leishmaniasis in parts of Ethiopia. Although most health systems rely on passive case detection for finding cutaneous leishmaniasis, active models should be explored. Screening by first-line health workers on the basis of well-defined risk factors, such as history of travel to foci of disease, skin lesions of more than 2 weeks' duration, male sex, profession associated with farming or visiting the jungle, and referral to centres where confirmation is possible may contribute to better control of cutaneous leishmaniasis.

Recommended case definitions for visceral and cutaneous leishmaniasis and PKDL are listed in Annex 3.

\subsection{Control of reservoir hosts}

Control of reservoir hosts has been recommended as a component of control strategies for zoonotic visceral and cutaneous leishmaniasis. For the control of anthroponotic leishmaniasis, an effective strategy for case detection, diagnosis and appropriate treatment of patients with clinical forms of leishmaniasis is a central pillar. In each situation, the actions to be taken depend on the local conditions and on the resources and knowledge available.

\subsubsection{Humans as reservoir hosts}

Humans are considered to be the sole proven reservoir of $L$. donovani infection in areas endemic for visceral leishmaniasis in Bangladesh, northern and eastern India and Nepal, and an important reservoir in the Sudan and East Africa. Similarly, L. tropica is considered to depend on humans for its survival, at least in long-established endemic foci. There are few xenodiagnostic data from India on the infectiousness to sandflies of patients with kalaazar and PKDL. As PKDL patients may continue to have symptoms for years in the absence of systemic illness and treatment, they probably constitute the main interepidemic reservoir. Leishmania-HIV coinfected patients are highly infectious to sandflies, and an increase in coinfection rates in an area is likely to increase the effective infection reservoir. The potential infectiousness of humans with asymptomatic $L$. donovani and L. tropica infection has unknown implications for control efforts.

Active case detection, surveillance and effective treatment, accompanied by measures for preventing reinfection, depending on the coverage achieved, should reduce or eliminate the parasite load and reduce transmission. The use of insecticide-treated bednets and other materials by patients with kala-azar and PKDL or with chronic L. tropica skin lesions may also decrease the likelihood that sandflies will feed on infected individuals (see section 3.5). 
Control of the canine leishmaniasis reservoir is a complex undertaking, which should be tailored to the local situation. The elimination of stray and feral dogs is justified for many reasons connected with health, the environment and conservation. The existence of zoonotic visceral leishmaniasis provides additional justification.

Before control activities begin, the distribution and frequency of the infection in dogs should be determined. Mass screening of domestic dogs is usually done by serological examination (ELISA, IFAT). At the same time, each dog can be examined clinically for skin alterations, onychogryphosis, lymph node enlargement and wasting. Less than half of infected dogs show signs of leishmaniasis, but a large proportion of asymptomatic infected dogs have been shown to be infective to sandflies by xenodiagnosis. Teams in charge of screening should take precautions against infection by Echinococcus granulosus (individual hygiene and protection) and by rabies virus (immunization). Ideally, all symptomatic or seropositive dogs should be eliminated; however, screening and mass culling of seropositive dogs has not proved uniformly effective in control programmes (for example, in Brazil). The suboptimal effectiveness has been attributed to delays between serology and culling, poor sensitivity of serological tests to identify the most infectious dogs and, mainly, only partial coverage of the infected canid population.

In several Mediterranean countries, euthanasia of infected domestic dogs is reserved for special cases, such as drug resistance, recurrent relapse or dangerous epidemiological situations. Most veterinarians prefer to manage canine leishmaniasis by giving antileishmanial treatment, while watching attentively for relapses. The medicines used primarily for treatment of human disease (see section 3.2.2) should not be used for treatment of canine leishmaniasis because of their low parasiticidal efficacy in this host and the potential for promoting parasite resistance. Alternative medicines, such as the leishmaniostatic allopurinol, should be used. A large percentage of treated dogs recover their infectivity to sandflies a few months after chemotherapy, despite clinical healing, thereby hiding the epidemiological problem of the source of infection.

Use of topical insecticides with proven efficacy against sandfly bites (deltamethrin- impregnated collars or permethrin-based spot-on formulations) has been shown to reduce the incidence of visceral leishmaniasis in dogs significantly (for example in Brazil, Italy and Tunisia) and humans (Islamic Republic of Iran), depending on the level of endemicity in a given area (see below). This approach has not, however, been tested in mass campaigns and cannot be endorsed as an alternative for interrupting transmission. 
Several canine Leishmania vaccines are under development or are licensed (see section 3.2.5). These vaccines were designed primarily to reduce severe manifestations of visceral leishmaniasis in dogs, and their potential impact on transmission is still unknown. Before such vaccines can be used to control zoonotic visceral leishmaniasis, phase- 3 studies must be conducted to assess their effectiveness to prevent transmission.

Legal measures can help to control the disease. Compulsory certification by veterinarians of the noninfective state of animals moving from one place to another would be helpful; however, the fact that many forms are asymptomatic or have a long incubation period limits the effect of such measures. In northern Europe, cases of canine leishmaniasis occur regularly in which the first manifestations are seen several years after a stay in an endemic zone. Registration and licensing of dogs are helpful but sometimes impracticable.

Control of canine visceral leishmaniasis could, in some countries, be associated with campaigns against rabies, which include (1) dog registration, to obtain useful information on the composition of the canine population and its dynamics; (2) evaluation of the health status of the canine population and regular veterinary surveillance; (3) access to serological diagnosis, medicines, topical insecticides and culling; and (4) mobilization of community participation (owners' responsibility).

\subsubsection{Wild animal reservoir hosts of Old World cutaneous leishmaniasis}

Rhombomys opimus (the great gerbil) is readily identifiable by its burrow system and distinctive morphology. Burrows can be found by combined aerial and ground surveys. An inexpensive, remarkably effective, economically viable approach is to destroy the burrow systems by ploughing with a subsoil plough followed by planting. Another approach is to poison the gerbils with zinc phosphide (12\%) mixed with wheat grains and vegetable oil $(2.3 \%)$. The grain baits must be introduced into the entrance of every three to four holes to a depth of at least $10 \mathrm{~cm}$. The anticoagulant dicoumarol has a synergistic effect with zinc phosphide; burrows are first treated with the anticoagulant, followed 5-7 days later by zinc phosphide. These compounds should be applied only by trained personnel and cannot always be applied to an entire focus, in which case a protective ring with a radius of $2-3 \mathrm{~km}$ should be established around human settlements.

Zinc phosphide is very toxic to other animals and to humans; therefore, treated grain should be inserted into the burrows to avoid damage to nontarget organisms. Total elimination of $R$. opimus can be achieved only in areas where reinvasion can be prevented by physical barriers such as canals or large agricultural fields. Temporary control, by clearing rodents from part of an area, can be achieved in unirrigated territories but not in oases or irrigated areas. 
Sound environmental planning and sanitation of foci should be prerequisites of agricultural development projects.

Burrows of Psammomys obesus (the fat sand rat) are readily identifiable by the halophytic vegetation around them and by the remnants of plant materials at the entrances. Methods for control of this rodent have not yet been designed. Grain treated with zinc phosphide is ineffective, as $P$. obesus does not eat grain, while anticoagulants are effective but too expensive. Other methods that may be tried include clearance of halophytic vegetation and physical destruction of the burrows (deep ploughing) followed by reforestation with locally appropriate trees to inhibit regrowth of Chenopodiaceae. A viable option is strictly to control the growth of these plants; destruction of wild Chenopodiaceae (e.g. Athrocnemum, Salicornia, Suedia) must be limited to selected protected areas, up to $1-2 \mathrm{~km}$ from dwellings, as these plants are often part of natural pasturage. The numbers of Psammomys can be estimated by counting active burrows or by direct observation in the early morning or evening, as the animals are active diurnally. Environmental changes due to agricultural development will, in the long term, lead to control of this rodent. In certain areas, however, Psammomys is a protected species.

Meriones spp. (jirds) are difficult to identify morphologically or by the appearance of their burrows, and specific training is required. Control measures have, however, been developed for agricultural purposes; anticoagulants and zinc phosphide are effective. In settlements where $M$. shawi is the reservoir host, a single worker with an applicator for introducing poisoned grain into burrows could control Meriones. In zones where there are human dwellings, regular collection of household rubbish, filling in of rubbish pits and mechanical destruction of burrows followed by land use are effective. The toxicity of zinc phosphide to nontarget animals must be re- emphasized (see above).

Control of hyraxes around villages may reduce the transmission of East African cutaneous leishmaniasis caused by L. aethiopica. Elimination of hyraxes within $1 \mathrm{~km}$ of settlements is thought to be effective in reducing transmission; it is not recommended that these animals be destroyed over a wider area. Hunters frequently trap and hand-catch hyraxes, but these methods are inefficient. As reinvasion is likely, control must be continuous. In some countries, hyraxes are protected animals.

\subsubsection{Wild animal reservoir hosts of New World cutaneous leishmaniasis}

Few rational measures for the control of reservoir hosts are available in the New World. No information is available on practical measures for controlling edentates; however, capture of sloths should be feasible when they descend from trees to defaecate. Control of Didelphis marsupialis (opossum) could 
be achieved in urban and semi-urban areas by trapping; baited pitfall traps could be used. This measure could be of great value in disturbed primary forest. No control method is currently available against procyonids or arboreal or ground sylvatic rodents.

An integrated environmental management approach might be effective, combining clearance of primary forest around villages and spraying of the cleared areas with insecticides to remove both the reservoir hosts and the vector, thus creating a vector- and reservoir-free zone around villages.

\subsection{Vector control}

\subsubsection{General considerations}

The aim of a vector control programme is to reduce or interrupt transmission of disease. An effective strategy for reducing human leishmaniasis is to control sandfly vectors, especially in domestic and peridomestic transmission habitats. A number of control methods are available, including chemicals, environmental management and personal protection. Although some methods can have a strong independent effect on sandfly populations, it is highly recommended that sandfly control involve more than one method, in an integrated vector management approach. Such a package depends on proper understanding of the local epidemiology of leishmaniasis (including whether transmission is anthroponotic or zoonotic) and detailed knowledge of the vector species involved, its habitats (peridomestic or sylvatic), flight range, host feeding preferences, resting sites, circadian rhythms and seasonality (see section 5).

The planning of integrated vector management requires an initial assessment of the ecology of the area, formulation of operational targets, choice of proper methods and a monitoring and evaluation scheme. Moreover, rational decision-making for the optimal use of resources and adequate institutional arrangements, including a regulatory framework, are needed. Implementation of integrated vector management requires decision-making and quality assurance procedures that can be applied at the lowest administrative level in the health system. The cost-effectiveness and community acceptability of the control measures are central to integrated vector management. All such programmes should include a strong component of social mobilization, with sufficient time and resources dedicated to informing communities and enhancing their participation.

Leishmaniases control has often been integrated with that of other vectorborne diseases. For example, after intensive attempts to eradicate malaria in the 1950s and 1960s by indoor spraying with DDT, the prevalence of leishmaniasis fell dramatically in many countries. In this approach, integrated 
vector management programmes combine interventions and resources and target several vector-borne diseases (e.g. malaria, dengue, filariasis) in one area. Research and development in vector control is essential, and control policy should keep pace with technological advances.

\subsubsection{Methods}

The approach to sandfly control depends on the behaviour of the target vector, which may be strongly endophilic, peridomestic or sylvatic. Endophilic species can be attacked by spraying insecticide on indoor walls. For peridomestic species, outer walls and animal accommodations should also be sprayed. Sylvatic species have been attacked by spraying trees that are the resting sites of some Neotropical species, although this may not be costeffective. The risk for infection in settlements in the forests can be reduced by clearing trees and bushes over a radius of at least $1 \mathrm{~km}$ around houses.

\section{Chemical control}

The main methods for controlling sandflies with insecticides are indoor residual spraying, spraying of resting sites of sylvatic species, use of insecticide-impregnated materials such as bednets and curtains, and pyrethroidimpregnated dog collars. In selecting the insecticide for indoor residual spraying or impregnation of nets, consideration should be given to their safety for humans and the environment, their efficacy (including duration of effectiveness), the cost-effectiveness, the acceptability and availability of goodquality products and the capacity and resources for safe, effective application and disposal of waste. The WHO Pesticide Evaluation Scheme (WHOPES) provides a list of WHO-recommended insecticides and dosages for indoor residual spraying. ${ }^{6}$

Indoor residual spraying is one of the main means for controlling endophilic sandfly vectors and should be targeted to localities with active transmission (focal spray). Therefore, good knowledge of the epidemiology of leishmaniasis and local vector behaviour and ecology is needed. Its level of effectiveness depends on the class of insecticide used, the susceptibility of sandflies to the insecticide, the type of surface treated, the dosage and method of application and overall coverage. When exophilic or peridomestic sandfly species are involved, outer surfaces of domestic animal shelters and structures close to such dwellings (potential sandfly resting sites) must be sprayed. Sustaining a high coverage rate is essential for long-term control, and this requires a well-organized programme, including technical guidelines with standard operating procedures, management, efficient logistics, supervision, monitoring and evaluation of efficacy. Noncompliance with operational guidelines

\footnotetext{
6 http://www.who.int/whopes/insecticides_iRS_malaria_09.pdf.
} 
is a waste of money and may moreover lead to environmental damage. Quality assurance systems should be established as part of every indoor residual spraying programme. In the visceral leishmaniasis elimination initiative on the Indian subcontinent, a vector control monitoring toolkit has been prepared for this purpose. ${ }^{7}$

Many classes of insecticide can be used in indoor residual spraying, including organochlorines (e.g. DDT), organophosphates (e.g. malathion), carbamates (e.g. propoxur) and synthetic pyrethroids (e.g. deltamethrin and $\lambda$-cyhalothrin). Malathion will potentially be included in a paint formulation (polyvinyl acetate-based suspension of malathion) for use against $L u$. longipalpis.

The spectrum of susceptibility of sandflies to the range of insecticides used in vector control programmes is not completely known. Resistance to organochlorine insecticides has been reported (e.g. resistance of P. papatasi and $P$. argentipes to DDT in India), and sandflies may have also developed resistance to malathion and pyrethroids in areas where these insecticides have been used for a long time, such as concurrently for malaria control. The choice of insecticide should be strictly regulated at national level, as the environmental legislation of some countries does not allow use of certain classes of insecticide. Recommendations for alternative insecticides should therefore be included in policies.

Insecticides should be rotated at appropriate intervals to prevent the emergence of resistance. All control programmes should include studies of susceptibility before the selection of insecticides, and resistance should be monitored at sentinel sites during the programme. Standard protocols for resistance monitoring and further information are available. ${ }^{8}$

Insecticide-treated nets are an effective, relatively cheap, sustainable method for sandfly control. The term covers both nets that are impregnated at regular intervals and long-lasting nets, in which insecticide is incorporated or coated on the fibre and which remain effective for 2-3 years. (See WHOPES for further information.) Under ideal conditions, insecticide-treated nets with people sleeping under them act as baited traps that kill sandflies. The synthetic pyrethroids used to treat nets should combine the properties of low-to-moderate mammalian toxicity, low volatility and high insecticidal activity. Trials of insecticide-treated nets in Afghanistan and the Syrian Arab Republic demonstrated protective efficacy against cutaneous leishmaniasis, and observational studies in Bangladesh, Nepal and the Sudan suggest a protective effect against visceral leishmaniasis. Several community trials in areas en-

\footnotetext{
7 WHO/SEARO. Indicator toolkit for the visceral leishmaniasis elimination initiative, 2010.

8 http://www.who.int/whopes/resistance/en/.
} 
demic for visceral leishmaniasis also showed reductions in vector density in clusters where insecticide-treated nets were used. The effectiveness of longlasting nets on reducing clinical outcomes in visceral leishmaniasis (infection, disease) is being evaluated in several countries.

Acceptability and human sleeping behaviour are critical to the effectiveness of insecticide-treated nets. It is often argued the nets are uncomfortable in the hot season due to lack of ventilation, but this idea stems from the false conception that nets must be of small mesh in order to protect against sandflies. Field studies show that insecticide-treated nets of normal mesh size are effective, and several studies have shown good acceptability in endemic areas, as the nets confer privacy and protect against nuisance from other insects. Screening of windows with insecticide- treated materials or impregnated curtains significantly reduced the numbers of sandflies entering houses in studies in Burkina Faso, Italy, the Sudan and the Latin American region.

Another method for control of cutaneous and zoonotic visceral leishmaniasis is the use of dog collars impregnated with pyrethroid insecticides. This method reduced the incidence of visceral leishmaniasis in children in a community trial in the Islamic Republic of Iran. In Brazil, their use under programme conditions revealed many operational problems, such as loss of collars. Pyrethroid insecticides can also be applied on dogs as shampoos, spotons and lotions, and these are being evaluated.

\section{Environmental management}

Environmental management results in a reduction in sandfly-human contact or sandfly populations through interventions in ecological niches. Examples are relocation of human settlements away from sandfly habitats and physical modification of the habitats. Environmental management measures must be preceded by careful studies of local ecology and the environmental impact. Physical modification of $P$. papatasi sandfly breeding and resting sites by destruction of the burrows of the great gerbil (R. opimus) was used successfully in the Central Asian republics of the former Soviet Union. Similarly, in Colombia, French Guiana and Panama, clearing of forests around villages and settlements effectively reduced or eliminated vector-human contact and Leishmania transmission. Actual or potential sandfly breeding sites, such as rubble and rubbish tips, can be eliminated in sanitation programmes involving the local community, especially in urban areas (e.g. Lu. whitmani in Brazil). It is important that any modification of vector habitats take into account environmental conservation and not create local ecological conflicts.

It is recommended that people entering or living in highly endemic foci use personal protection measures to avoid bites by sandfly vectors of leishmani- 
asis. These measures include avoiding times and places of sandfly activity and application of insect repellents on exposed skin.

\subsubsection{Entomological monitoring and evaluation of vector control operations}

A well-designed scheme for monitoring and evaluating an integrated vector management programme should be prepared before any sandfly control operation is launched. The scheme should include clear definitions of the process, output and outcome indicators of the programme. The evaluation scheme should include methods to assess both the short- and long-term effects of the control measures on the vector population. A set of standard indicators is included in the tool kit prepared by WHO/TDR.

Routine indicators of quality include:

- the performance of people conducting indoor residual spraying (or dipping insecticide-treated nets), by observation;

- the accuracy of spraying, i.e. the percentage of chemical on the wall in comparison with the target concentration (filter paper technique for indoor residual spraying or analysis of insecticide-treated nets);

- bioassay of the effectiveness of indoor residual spraying or insecticidetreated nets (see WHOPES site);

- vector density, monitored by knock-down catches of sandflies resting indoors, light trapping, quantitative sticky-paper traps or standardized active catches of day-resting sandflies, depending on the sandfly species and its habits; and

- acceptability, as outlined in the TDR toolkit.

Evaluation of the effectiveness of interventions on transmission should include studies with epidemiological end-points in humans (infection, disease) or studies of the effect on infection rates in sandflies.

\subsection{Epidemic response}

Epidemics are characteristic of both cutaneous and visceral leishmaniasis. Long- standing endemic foci can erupt into epidemics, or new foci can appear where leishmaniasis has not previously been reported. Large epidemics of anthroponotic visceral leishmaniasis ( $L$. donovani) and cutaneous leishmaniasis ( $L$. tropica) occur. Outbreaks of zoonotic visceral leishmaniasis $(L$. infantum) and cutaneous leishmaniasis (L. major, L. braziliensis, L. mexicana. L. aethiopica) also occur but are less frequent than for anthroponotic species. The emergence of an epidemic is difficult to predict; factors that can be involved include changes in vector habitat (reforestation), mass movements of people (immigration, seasonal migration, war) and decreased immunity (malnutrition). 
During epidemics in nonendemic areas, children and adults are more equally affected than in endemic areas, as they are all nonimmune. In stable endemic areas, infections are commoner in children or displaced persons, as most adults will have had a clinical or subclinical infection in the past. The control measures used in epidemics must be based on previous observations and adapted to local conditions.

\subsubsection{Rapid assessment}

Certain measures should be taken urgently.

- Establish the etiology of the outbreak, including identification of the parasite species and the vector.

- Confirm the outbreak by comparing current and previous (ideally for the previous 5 years) incidence of the disease, while allowing for seasonal variation, past epidemics and potential changes in completeness of reporting due to alterations in local conditions (e.g. insecurity affecting access to health- care facilities). Clustering of cases over a short period may also indicate an outbreak. Rumours and poor clinical skill in remote areas may exaggerate the epidemic; alternatively, poor diagnosis may result in underestimation of the outbreak. Therefore, laboratory confirmation by microscopy (visceral and cutaneous leishmaniasis) or serology (visceral leishmaniasis) is essential.

- Collect information on the movements of patients and conduct investigations in the areas visited to determine whether populations have been affected, as visceral leishmaniasis has a relatively long incubation period (typically 2-6 months), and transmission may not be endemic in the location where clinical cases are identified.

- Use a standard case definition for suspect, probable and confirmed cases (see Annex 3).

- Estimate the extent of the epidemic by systematic collection of epidemiological data (number of cases, age, gender, geographical origin, time), with the standard case definition.

- Assess the availability and quality of diagnostic and treatment services in health facilities in the affected area in order to identify service delivery gaps.

- Monitor essential supplies of diagnostics, medicines and insecticidetreated materials at national, regional and local levels to permit timely supply management and ensure availability.

- Use prompt, regular reporting mechanisms and protocols for standardized data capture, analysis, reporting and dissemination to allow effective decision- making and coordination of response activities.

- Conduct retrospective or prospective studies to improve understanding of the evolution of the epidemic. Precise mapping is necessary to es- 
tablish the exact geographical area affected, and data are needed on the origin and severity of the outbreak.

Monitoring and evaluation are covered in section 6.5.

\subsubsection{Epidemic preparedness}

An effective emergency response to a leishmaniasis outbreak requires advance planning.

- Reinforce the surveillance system at the start of the traditional peak season, and establish criteria for alert and epidemic thresholds.

- Establish and distribute case definitions and treatment protocols.

- Ensure that all health facilities have minimum stocks of basic diagnostic and treatment supplies, algorithms, protocols and data collection tools at the beginning of the outbreak season.

- Before the anticipated outbreak season, identify and define the responsibilities of members of the leishmaniasis outbreak task force at national, regional and district levels; and assess their capacities for medical response, surveillance and training.

\subsubsection{Outbreak response}

- Ensure that funds are available for immediate purchase of essential supplies and transport and e.g. staff and training; include a system for tracking and auditing funds.

- Ensure the availability of essential supplies, including medicines (antileishmanial and for management of opportunistic infections in visceral leishmaniasis), rapid diagnostic tests, laboratory and parasitological diagnostic equipment, surveillance and monitoring tools (including disease-specific forms).

- Train hospital and health centre staff in diagnostic and treatment algorithms and protocols and the basic epidemiology of leishmaniasis. Train community health workers in community sensitization and active case finding and referral, with a simplified clinical case definition.

- Decentralize services as much as possible, as the key to effective outbreak management is adequate access to health facilities that provide early diagnosis and treatment. Furthermore, decentralization of treatment services to health centres and clinics may be essential to prevent overloading hospitals and to reduce the risk for outbreaks of opportunistic infections among immunocompromised patients with visceral leishmaniasis living in crowded conditions.

- Use a 'hub-and-spoke' approach to detect the progression of an epidemic, in which mobile or short-term treatment centres can be opened 
and closed swiftly as needed, as the movement of epidemics is not predictable, even though the overall at-risk area may be known.

- Recognize the usefulness of peripheral health posts or community health workers in identifying and referring clinically suspect cases to a health facility for confirmatory testing and treatment.

- When there is high mortality from visceral leishmaniasis, conduct active case- finding in the areas from which the victims came, as a high case fatality rate is a result of advanced disease and indicates that patients had difficulty or delay in accessing treatment.

- Provide information to all levels (health centres, hospitals, diagnosis and treatment centres and local communities) in messages that contain clear, simple instructions to the population at risk to consult a health centre at an early stage of the disease, the locations of diagnosis and treatment centres, epidemiological data and practical measures for prevention.

- Implement vector control on the basis of a sound understanding of the local epidemiological cycle and vector behaviour. Because of the relatively long incubation period, vector control will reduce an epidemic only if transmission is ongoing during the outbreak. Apply vector control measures (indoor or outdoor residual spraying, distribution of longlasting insecticide-treated nets) immediately before the next transmission season.

- Treat patients promptly and effectively, especially those with anthroponotic cutaneous and visceral leishmaniasis, as this may shorten the epidemic. Cases of PKDL should be treated to prevent transmission of visceral leishmaniasis.

- Use an effective network of communication and transparent decisionmaking to facilitate coordination in the outbreak response team, including nongovernmental and community organizations.

- Mobilize the considerable resources of international technical expertise to assist where needed in all these measures.

- Ensure multisector involvement.

\subsection{Socioeconomic aspects of leishmaniasis control}

Leishmaniasis is related to poverty, affecting 'the poorest of the poor'. In poor states such as Bihar in India, the visceral form of leishmaniasis affects families in the lowest income groups, who live on less than US\$ 1 per day. The relation between leishmaniasis and poverty is complex: while poverty increases the risk for leishmaniasis and aggravates disease progression, leishmaniasis itself leads to further impoverishment of the family due to catastrophic health expenditure, income loss and death of wage earners. Control 
strategies that do not take into account the socioeconomic context of this disease will be difficult to sustain in the long term.

\subsubsection{Social determinants of risk}

\section{Poverty}

Poverty is associated with ecological factors that increase the risk for infection due to proliferation of the vector or increased human-vector contact. In areas of anthroponotic peridomestic transmission, such as the Indian subcontinent, proliferation of the vector is enhanced by poor housing conditions, such as damp earthen floors, which prolong survival of the vector, and cracked mud walls, which provide the vector with daytime resting places. Sleeping outside or on the ground may increase exposure to sandflies. In South America, visceral leishmaniasis used to be a rural disease, enhanced by deforestation and intrusion of immunologically naive populations into areas with sylvatic transmission cycles. More recently, it is becoming a periurban disease, linked to migration of poor rural families to major cities. Poor environmental sanitation in these settings and erratic rubbish collection may increase the risk for leishmaniasis.

Poverty also worsens clinical outcomes in leishmaniasis, as malnutrition and anaemia increase the severity of the disease. Leishmaniasis also aggravates poverty. Even when households do not have to pay the direct medical costs of care, such as antileishmanial medicines, the economic impact of the disease includes direct nonmedical costs (e.g. transport) and income loss for patients and their families due to absence from work. Several studies of the cost or burden of illness have addressed the economic burden of leishmaniasis on households. On the Indian subcontinent, the median total expenditure by a patient on visceral leishmaniasis treatment was 1.2 to 1.4 times the annual per capita income.

\section{Gender}

Clinical disease in men is reported more frequently than that in women in most endemic countries. Although this difference could be due to more frequent exposure of males than females, it is also due to underdetection of disease in women in traditionally male-dominated societies. Communitybased studies in Bangladesh showed that the incidence of visceral leishmaniasis was about equal, but the case fatality rate was threefold higher in women than men. The difference was attributed to higher rates of malnutrition and anaemia in women and longer delays in seeking care.

Drug policy should take account of gender; for instance, women of childbearing age must not be prescribed miltefosine if they do not have adequate contraception, as the disease has potential effects on pregnancy. Disfigure- 
ment caused by cutaneous leishmaniasis or PKDL leads to stigma and isolation and is a barrier to marriage, especially for girls.

\section{Access to health care}

The weakness of health systems in hyperendemic areas is one of the major factors in sustained transmission of anthroponotic visceral and cutaneous leishmaniasis. A substantial number of cases are either not diagnosed or only after a long delay, increasing the risk for a fatal outcome (visceral leishmaniasis) or infection of families. In many countries, an important number of patients with visceral or cutaneous leishmaniasis seek care from private providers, who do not always respond adequately to the syndrome. More formal involvement of private practitioners in control programmes should be explored, as was done for instance for tuberculosis.

\section{Sociocultural barriers}

Leishmaniasis typically clusters in marginalized communities, such as the mushar in India. It is important to increase the awareness of communities about the disease and its control. Specific, adapted messages of health education should be developed. Community participation is essential in order to maximize the effect of control strategies, including case detection, vector control and control of animal reservoirs. Good dialogue with communities must be established.

\subsubsection{Cost-effectiveness of control measures}

\section{Diagnosis and treatment}

Formal analyses have been conducted of cost-effectiveness, based on decision analytical models, to compare alternative options in diagnostics and treatment for visceral leishmaniasis. It was found that the cost-effectiveness depends more on the cost of treatment than on that of testing. A strategy based on the rK39 rapid test or the direct agglutination test is more efficient than one based on bone-marrow or lymph node aspiration, as the sensitivity of a test is paramount in this fatal disease.

A recent study of the cost of medicines for India based on international drug prices (see Annex 6) and anthropometric data from a specialized treatment centre in Bihar showed that paromomycin is the cheapest option (US\$ 7.4 per patient) and liposomal amphotericin B the most expensive (US\$ 162-229 per patient). Treatment with miltefosine would cost US\$ 119 per patient at the private company price and US\$ 64-75 at the WHO-negotiated price. These calculations do not include other direct or indirect costs and may differ widely from country to country. For example, in Nepal, a full course of treatment with miltefosine can cost up to US\$ 150 per patient for medicines only. (In 
developed countries, the drug price can be 10 to 50 times higher than the preferential price.)

Short regimens, including combination therapies, can reduce the cost to the public health system and patients by reducing the duration of treatment. Coadministration of miltefosine with paromomycin for 10 days or a combination of single-dose liposomal amphotericin B with paromomycin were competitive in terms of cost- effectiveness with short-course monotherapy on the Indian subcontient (depending on the drug costs). Short regimens also reduce the period of economic inactivity of patients. Ultra-short regimens, such as single-dose liposomal amphotericin B, are therefore the most advantageous.

Drug prices are, however, rapidly changing, and policy-makers need updated cost information to make informed decisions on the best treatment options. Although the cost of cutaneous leishmaniasis treatment is usually lower than that for visceral leishmaniasis (see Annex 6), formal data on the costs of diagnosis and treatment of cutaneous leishmaniasis or New World visceral leishmaniasis are not available.

\section{Vector and reservoir control}

No information is available on the cost-effectiveness of measures for vector and reservoir control.

\subsubsection{Access to medicines and diagnostics}

Access to medicines for the treatment of visceral, cutaneous and mucocutaneous leishmaniases is problematic in the poverty-stricken countries that have the highest burden of cases. Although many efforts have been made by WHO, medical nongovernmental organizations and manufacturers to improve access to medicines for leishmaniasis, problems persist. In the context of weak health systems in many Leishmania-endemic countries, a number of factors contribute to lack of access to medicines.

- The medicines are relatively expensive and are often unaffordable, despite price reductions negotiated by WHO.

- National treatment protocols often do not reflect the latest developments, and few or no medicines for leishmaniasis are included in national essential drug lists.

- Medicines for which preferential prices have been negotiated in lowand middle-income countries are not always registered by the companies where they are needed, as there is no profitable market. If medicines are not registered, special permission for their import is required. Registration is also often lacking in countries with very few cases. Medical practitioners in these countries experience great difficulty in obtaining the small quantities of medicines needed ad hoc. 
- The supply of medicines is not continuous. Most antileishmanial medicines are produced by only a single manufacturer. Problems with quality, low production capacity and lack of an adequate forecast of needs (resulting in long lead times for drug orders) regularly cause stock ruptures in endemic countries. There are no central buffer stocks that can be accessed in such cases, and there is no platform for indicating drug needs; therefore, the quantities needed globally cannot be estimated and drug production cannot be appropriately planned.

Overall monitoring of access to leishmanial medicines should be strengthened, with pricing, registration and global needs taken into account. Moreover, the regulation of drug policies and quality assurance should be strengthened at all levels. Uncontrolled access to medicines (e.g. over-thecounter availability of miltefosine) could lead to misuse, suboptimal treatment and, in the long term, drug resistance. The use of counterfeit medicines (toxic batches of antimonials, fake miltefosine) has led to several avoidable deaths in the past.

The supply of diagnostics for visceral leishmaniasis is marked by similar problems, as the market is relatively small and not profitable. Moreover, diagnostic kits of uncertified quality circulate in endemic regions.

\subsubsection{Public-private partnerships}

Over the past few decades, a number of initiatives have been set up to address various global health crises, and pharmaceutical companies have been providing support by donating or subsidizing drugs or by developing drugs for neglected diseases. During the past 10 years, several new drugs have been developed for leishmaniasis, due to increased collaboration between international organizations, foundations, private pharmaceutical companies, governments and universities. There is no drug donation programme for leishmaniasis, but several companies have agreed to make antileishmanial drugs available at subsidized prices after negotiation with WHO (see Annex 6). 


\section{Burden of leishmaniases}

\subsection{Geographical distribution by country}

The distribution of the leishmaniases by country or territory is shown in Table 4 for the Old World and Table 5 for the New World.

Table 4.

Distribution of Old World leishmaniasis by country or territory, 2009

\begin{tabular}{|c|c|c|c|c|}
\hline $\begin{array}{l}\text { Country or } \\
\text { territory }\end{array}$ & Species & $\begin{array}{l}\text { Clinical } \\
\text { form }\end{array}$ & $\begin{array}{l}\text { Proven or suspected } \\
\text { Phlebotomus vector }\end{array}$ & $\begin{array}{l}\text { Proven or } \\
\text { suspected animal } \\
\text { reservoir }\end{array}$ \\
\hline \multirow[t]{3}{*}{ Afghanistan } & L. tropica & $\mathrm{ACL}$ & P. sergenti & Human \\
\hline & L. major & $\mathrm{ZCL}$ & $\begin{array}{l}\text { P. papatasi, } P \text {. } \\
\text { caucasicus }\end{array}$ & $\begin{array}{l}\text { R. opimus, } \\
\text { Meriones spp. }\end{array}$ \\
\hline & L. infantum & VL & Unknown & Dog \\
\hline Albania & L. infantum & ZVL, CL & $\begin{array}{l}\text { P. neglectus, } P \text {. } \\
\text { perfiliewi, } P \text {. tobbi }\end{array}$ & Dog \\
\hline \multirow[t]{3}{*}{ Algeria } & L. infantum & ZVL, CL & $\begin{array}{l}P \text {. longicuspis, } P \text {. } \\
\text { perfiliewi, } P \text {. } \\
\text { perniciosus }\end{array}$ & Dog \\
\hline & L. killicki & $\mathrm{CL}$ & Unknown & \\
\hline & L. major & ZCL & P. papatasi & $\begin{array}{l}\text { Psammomys } \\
\text { obesus }\end{array}$ \\
\hline Armenia & L. infantum & ZVL, CL & $\begin{array}{l}P . \text { kandelakii, } P \text {. } \\
\text { balcanicus }\end{array}$ & Dog \\
\hline \multirow[t]{3}{*}{ Azerbaijan } & L. infantum & ZVL & $\begin{array}{l}\text { P. kandelakii, } P \text {. } \\
\text { transcaucasicus }\end{array}$ & Dog, V. vulpes \\
\hline & L. major & $\mathrm{ZCL}$ & P. papatasi & R. opimus \\
\hline & L. tropica & $\mathrm{ACL}$ & P. sergenti & Human \\
\hline Bangladesh & L. donovani & AVL, PKDL & $P$. argentipes & Human \\
\hline
\end{tabular}




\begin{tabular}{|c|c|c|c|c|}
\hline $\begin{array}{l}\text { Bosnia and } \\
\text { Herzegovina }\end{array}$ & L. infantum & ZVL, CL & Unknown & Dog \\
\hline Bulgaria & L. infantum & ZVL, CL & Unknown & Dog \\
\hline Burkina Faso & L. major & $\mathrm{ZCL}$ & $\begin{array}{l}\text { P. duboscqi, } P \text {. } \\
\text { bergeroti }\end{array}$ & Unknown \\
\hline Buthan & L. donovani & $A V L$ & Unknown & Human \\
\hline Cameroon & L. major & $\mathrm{ZCL}$ & P. duboscqi & Unknown \\
\hline $\begin{array}{l}\text { Central African } \\
\text { Republic }\end{array}$ & L. infantum & VL & Unknown & \\
\hline \multirow[t]{2}{*}{ Chad } & Unknown & VL & P. orientalis & \\
\hline & L. major & ZCL & $\begin{array}{l}P . \text { duboscqi, } P \text {. } \\
\text { bergeroti }\end{array}$ & Unknown \\
\hline \multirow[t]{2}{*}{ China } & L. infantum & ZVL, CL & $\begin{array}{l}P . \text { chinensis, } P \text {. } \\
\text { alexandri, } P \text {. wui }\end{array}$ & $\begin{array}{l}\text { Dog, } N . \\
\text { procyonoides }\end{array}$ \\
\hline & L. donovani & $A V L$ & P. longiductus & Human \\
\hline $\begin{array}{l}\text { China (Province } \\
\text { of Taiwan) }\end{array}$ & Unknown & CL, DCL & P. kiangsuensis & \\
\hline Côte d'lvoire & Unknown & VL, CL & Unknown & \\
\hline Croatia & L. infantum & ZVL, CL & $\begin{array}{l}P . \text { tobbi, } P \text {. } \\
\text { neglectus }\end{array}$ & Dog \\
\hline \multirow[t]{2}{*}{ Cyprus } & L. infantum & ZVL, CL & P. tobbi & Dog \\
\hline & L. donovani & $\mathrm{VL}, \mathrm{CL}$ & Unknown & \\
\hline $\begin{array}{l}\text { Democratic } \\
\text { Republic of the } \\
\text { Congo }\end{array}$ & Unknown & VL, CL & Unknown & \\
\hline \multirow[t]{2}{*}{ Djibouti } & L. donovani & VL & Unknown & \\
\hline & Unknown & $\mathrm{CL}$ & Unknown & \\
\hline \multirow[t]{3}{*}{ Egypt } & $L$ infantum & ZVL & P. langeroni & Dog \\
\hline & L major & ZCL & P. papatasi & $\begin{array}{l}\text { Ps. obesus, } \\
\text { Gerbillus spp. }\end{array}$ \\
\hline & L. tropica & $\mathrm{CL}$ & Unknown & \\
\hline
\end{tabular}




\begin{tabular}{|c|c|c|c|c|}
\hline Eritrea & Unknown & VL, CL & Unknown & \\
\hline \multirow[t]{4}{*}{ Ethiopia } & L. aethiopica & $\begin{array}{l}\text { ZCL, DCL, } \\
M L\end{array}$ & $\begin{array}{l}P . \text { longipes, } P \text {. } \\
\text { pedifer, } P \text {. sergenti }\end{array}$ & $\begin{array}{l}\text { Procavia capensis, } \\
\text { Heterohyrax brucei }\end{array}$ \\
\hline & L. major & ZCL & P. dubosqui & $\begin{array}{l}\text { Arvicanthis } \\
\text { niloticus }\end{array}$ \\
\hline & L. tropica & $\mathrm{CL}$ & $\begin{array}{l}P . \text { sergenti, } P \text {. } \\
\text { saeveus }\end{array}$ & \\
\hline & L. donovani & VL, PKDL & $\begin{array}{l}P \text {. orientalis, } P \text {. } \\
\text { martini, } P \text {. celiae }\end{array}$ & \\
\hline France & L. infantum & ZVL, CL & $\begin{array}{l}P \text {. perniciosus, } P \text {. } \\
\text { ariasi }\end{array}$ & Dog, V. vulpes \\
\hline Gambia & L. infantum & $\mathrm{CL}$ & P. duboscqi & Dog \\
\hline \multirow[t]{2}{*}{ Georgia } & L. infantum & ZVL, CL & $\begin{array}{l}P \text {. kandelakii, } P \text {. } \\
\text { balcanicus, } P \text {. } \\
\text { halepensis }\end{array}$ & Dog \\
\hline & L. major & $\mathrm{CL}$ & Unknown & \\
\hline Ghana & L. major & $\mathrm{CL}$ & P. duboscqi & \\
\hline \multirow[t]{2}{*}{ Greece } & L. infantum & ZVL, CL & $\begin{array}{l}P \text {. neglectus, } P \text {. } \\
\text { perfiliewi, } P \text {. tobbi }\end{array}$ & Dog \\
\hline & L. tropica & $\mathrm{CL}$ & P. sergenti & \\
\hline Guinea & L. major & $\mathrm{CL}$ & P. duboscqi & \\
\hline Guinea-Bissau & L. major & $\mathrm{CL}$ & P. duboscqi & \\
\hline \multirow[t]{3}{*}{ India } & L. donovani & AVL, PKDL & P. argentipes & Human \\
\hline & L. major & $\mathrm{ZCL}$ & P. salehi, P. papatasi & M. hurrianae \\
\hline & L. tropica & $\mathrm{ACL}$ & P. sergenti & Human \\
\hline \multirow[t]{3}{*}{$\begin{array}{l}\text { Iran (Islamic } \\
\text { Republic of) }\end{array}$} & L. major & $\mathrm{ZCL}$ & $\begin{array}{l}P . \text { papatasi, } P \text {. } \\
\text { salehi, } P \text {. ansaril, } P \text {. } \\
\text { caucasicus }\end{array}$ & $\begin{array}{l}\text { R. opimus, } \\
\text { Meriones spp., } \\
\text { Tatera indica, } \\
\text { Nesokia indica }\end{array}$ \\
\hline & L. tropica & $\mathrm{ACL}$ & P. sergenti & Human \\
\hline & L. infantum & ZVL & P. kandelakii & Dog \\
\hline
\end{tabular}




\begin{tabular}{|c|c|c|c|c|}
\hline \multirow[t]{4}{*}{ Iraq } & L donovani & VL & P. alexandri & \multirow{4}{*}{ Human } \\
\hline & L. tropica & $A C L$ & P. sergenti & \\
\hline & L. major & $\mathrm{CL}$ & P. papatasi & \\
\hline & L. infantum & VL & Unknown & \\
\hline \multirow[t]{4}{*}{ Israel } & L. major & ZCL & P. papatasi & $\begin{array}{l}\text { Ps. obesus, } M \text {. } \\
\text { crassus, Microtus } \\
\text { socialis, N. indica }\end{array}$ \\
\hline & L. tropica & $\mathrm{CL}$ & $\begin{array}{l}P \text {. sergenti, } P \text {. } \\
\text { arabicus }\end{array}$ & $\begin{array}{l}\text { Human, Pr. } \\
\text { capensis }\end{array}$ \\
\hline & L. infantum & ZVL, CL & $\begin{array}{l}\text { P. syriacus, } P \text {. } \\
\text { perfiliewi, } P \text {. tobbi }\end{array}$ & Dog \\
\hline & L. donovani & $\mathrm{CL}$ & Unknown & \\
\hline Italy & L. infantum & ZVL, CL & $\begin{array}{l}P \text {. perniciosus, } P \text {. } \\
\text { perfiliewi, } P \text {. } \\
\text { neglectus, } P \text {. ariasi }\end{array}$ & Dog, V. vulpes \\
\hline \multirow[t]{3}{*}{ Jordan } & L. infantum & ZVL & Unknown & Dog \\
\hline & L. tropica & CL & $P$. sergenti & \\
\hline & L. major & ZCL & P. papatasi & $\begin{array}{l}\text { Ps. obesus, M. } \\
\text { libycus }\end{array}$ \\
\hline \multirow[t]{2}{*}{ Kazakhstan } & L. infantum & ZVL & $\begin{array}{l}P . \text { smirnovi, } P \text {. } \\
\text { longiductus }\end{array}$ & Dog \\
\hline & $L$ major & ZCL & $\begin{array}{l}P . \text { papatasi, } P \text {. } \\
\text { mongolensis }\end{array}$ & R. opimus \\
\hline \multirow[t]{4}{*}{ Kenya } & L. tropica & ZCL & P. guggisbergi & Pr. capensis \\
\hline & L. aethiopica & ZCL, DCL & $\begin{array}{l}P . \text { pedifer, } P \text {. } \\
\text { aculeatus }\end{array}$ & $\begin{array}{l}\text { Pr. capensis, } \\
\text { Dendohyrax } \\
\text { arboreous, } \\
\text { Cricetomys spp. }\end{array}$ \\
\hline & L. major & ZCL & P. duboscqi & $\begin{array}{l}\text { Tatera spp. } \\
\text { Aethomys spp., } \\
\text { Arvicanthis spp., } \\
\text { Meriones spp. }\end{array}$ \\
\hline & L. donovani, & VL, PKDL & $\begin{array}{l}P . \text { martini, } P \text {. celiae, } \\
P . \text { vansomerenae }\end{array}$ & \\
\hline
\end{tabular}




\begin{tabular}{|c|c|c|c|c|}
\hline Kyrgyzstan & L. infantum & ZVL, CL & P. longiductus & Dog \\
\hline Kuwait & L. major & $\mathrm{CL}$ & Unknown & \\
\hline Lebanon & L. infantum & $\mathrm{ZVL}, \mathrm{CL}$ & P. syriacus & Dog \\
\hline \multirow{3}{*}{$\begin{array}{l}\text { Libyan Arab } \\
\text { Jamahiriya }\end{array}$} & L. infantum & VL & Unknown & Dog \\
\hline & L. killicki & $\mathrm{CL}$ & Unknown & \\
\hline & L. major & $\mathrm{ZCL}$ & P. papatasi & $\begin{array}{l}\text { Ps. obesus, } \\
\text { Meriones spp. }\end{array}$ \\
\hline Macedonia & L. infantum & ZVL, CL & Unknown & Dog \\
\hline Malawi & Unknown & $\mathrm{CL}$ & Unknown & \\
\hline Mali & L. major & $\mathrm{ZCL}$ & P. duboscqi & \\
\hline Malta & L. infantum & ZVL, CL & P. pemiciosus & Dog \\
\hline \multirow[t]{2}{*}{ Mauritania } & L. major & $\mathrm{CL}$ & $\begin{array}{l}\text { P. duboscqi, } P \text {. } \\
\text { bergeroti }\end{array}$ & \\
\hline & L. infantum & VL, CL & Unknown & \\
\hline Monaco & L. infantum & ZVL, CL & P. pemiciosus & Dog \\
\hline Mongolia & L. major & $\mathrm{CL}$ & Unknown & \\
\hline Montenegro & L. infantum & ZVL & P. neglectus & Dog \\
\hline \multirow[t]{3}{*}{ Morocco } & L. major & $\mathrm{ZCL}$ & P. papatasi & $\begin{array}{l}\text { M. shawi, Ps. } \\
\text { obesus }\end{array}$ \\
\hline & L. tropica & $\mathrm{CL}$ & $\begin{array}{l}P \text {. sergenti, } P \text {. } \\
\text { chabaudi }\end{array}$ & Human, dog \\
\hline & L. infantum & ZVL, CL & $\begin{array}{l}P \text {. perniciosus, } P \text {. } \\
\text { ariasi, } P \text {. longicuspis }\end{array}$ & Dog \\
\hline Namibia & L. tropica & ZCL & P. rossi, P. grovei & Pr. capensis \\
\hline
\end{tabular}




\begin{tabular}{|c|c|c|c|c|}
\hline Nepal & L. donovani & AVL, PKDL & $P$. argentipes & Human \\
\hline \multirow[t]{2}{*}{ Niger } & L. major & ZCL & P. duboscqi & Unknown \\
\hline & Unknown & VL & Unknown & \\
\hline Nigeria & L. major & $\mathrm{ZCL}$ & P. duboscqi & Unknown \\
\hline \multirow[t]{2}{*}{ Oman } & L. infantum & ZVL & P. alexandri & Dog \\
\hline & L. major & $\mathrm{ZCL}$ & P. papatasi & Unknown \\
\hline \multirow[t]{3}{*}{ Pakistan } & L. tropica & $\mathrm{ACL}$ & P. sergenti & Human \\
\hline & L. major & $\mathrm{ZCL}$ & P. papatasi, P. salehi & $\begin{array}{l}\text { M. hurrianae, } R \text {. } \\
\text { opimus, } T \text {. indica }\end{array}$ \\
\hline & L. infantum & ZVL & Unknown & Dog \\
\hline \multirow[t]{3}{*}{ Palestine } & L.major & $\mathrm{ZCL}$ & P. papatasi & Ps. obesus \\
\hline & L. infantum & ZVL & $\begin{array}{l}P . \text { syriacus, } P . \\
\text { perfiliewi, } P \text {. tobbi }\end{array}$ & Dog \\
\hline & L. tropica & $\mathrm{ACL}$ & P. sergenti & Unknown \\
\hline Portugal & L. infantum & ZVL, CL & $\begin{array}{l}P \text {. perniciosus, } P \text {. } \\
\text { ariasi }\end{array}$ & Dog, V. vulpes \\
\hline Romania & L. infantum & ZVL & $\begin{array}{l}P . \text { neglectus, } P \text {. } \\
\text { perfiliewi }\end{array}$ & Dog \\
\hline \multirow[t]{4}{*}{ Saudi Arabia } & L. tropica & $\mathrm{CL}$ & P. sergenti & \\
\hline & L. infantum & ZVL & Unknown & Dog \\
\hline & L. major & $\mathrm{ZCL}$ & P. papatasi & $\begin{array}{l}\text { Meriones spp., Ps. } \\
\text { obesus }\end{array}$ \\
\hline & L. donovani & VL & Unknown & \\
\hline \multirow[t]{2}{*}{ Senegal } & L. major & ZCL & P. duboscqi & $\begin{array}{l}\text { A. niloticus, } T \text {. } \\
\text { gambiana, } \\
\text { Mastomys } \\
\text { erythroleucus }\end{array}$ \\
\hline & L. infantum & ZVL & Unknown & Dog \\
\hline
\end{tabular}




\begin{tabular}{|c|c|c|c|c|}
\hline Slovenia & L. infantum & VL, CL & P. neglectus & Dog \\
\hline Somalia & L. donovani & VL, PKDL & P. martini & \\
\hline South Africa & Unknown & $\mathrm{CL}$ & Unknown & \\
\hline Spain & L. infantum & ZVL, CL & $\begin{array}{l}P \text {. perniciosus, } P \text {. } \\
\text { ariasi }\end{array}$ & Dog \\
\hline Sri Lanka & L. donovani & VL, CL & Unknown & \\
\hline \multirow[t]{3}{*}{ Sudan } & L. donovani & $\begin{array}{l}\text { VL, PKDL, } \\
\text { ML }\end{array}$ & $\begin{array}{l}P \text {. orientalis, } P \text {. } \\
\text { martini }\end{array}$ & Human \\
\hline & L. infantum & ZVL & Unknown & Dog \\
\hline & L. major & ZCL, ML & $\begin{array}{l}\text { P. papatasi, } P \text {. } \\
\text { duboscqi }\end{array}$ & A. niloticus \\
\hline \multirow{3}{*}{$\begin{array}{l}\text { Syrian Arab } \\
\text { Republic }\end{array}$} & L. tropica & $\mathrm{ACL}$ & P. sergenti & Human \\
\hline & L. major & $\mathrm{ZCL}$ & P. papatasi & $\begin{array}{l}\text { Ps. obesus, } \\
\text { Meriones spp., } N \text {. } \\
\text { indica }\end{array}$ \\
\hline & L. infantum & ZVL & $\begin{array}{l}P \text {. galilaeus, } P \text {. } \\
\text { syriacus, } P \text {. tobbi, } P \text {. } \\
\text { halepensis }\end{array}$ & Dog \\
\hline \multirow[t]{2}{*}{ Tajikistan } & Unknown & $\mathrm{CL}$ & Unknown & \\
\hline & Unknown & VL & Unknown & \\
\hline Thailand & Unknown & VL & Unknown & \\
\hline \multirow[t]{3}{*}{ Tunisia } & L. infantum & ZVL, CL & $\begin{array}{l}P \text {. langeroni, } P \text {. } \\
\text { pemiciosus, } P \text {. } \\
\text { perfiliewi, } P \text {. } \\
\text { longicuspis }\end{array}$ & Dog \\
\hline & L. major & ZCL & P. papatasi & $\begin{array}{l}\text { Ps. obesus, } \\
\text { Meriones spp. }\end{array}$ \\
\hline & L. killicki & $\mathrm{CL}$ & Unknown & \\
\hline \multirow[t]{2}{*}{ Turkey } & L. infantum & ZVL, CL & $\begin{array}{l}P \text {. neglectus, } P \text {. } \\
\text { syriacus, } P \text {. tobbi, } P \text {. } \\
\text { alexandri }\end{array}$ & Dog \\
\hline & L. tropica & ACL & P. sergenti & Human \\
\hline
\end{tabular}




\begin{tabular}{|c|c|c|c|c|}
\hline \multirow[t]{3}{*}{ Turkmenistan } & L. major & ZCL & P. papatasi & R. opimus \\
\hline & L. tropica & $\mathrm{CL}$ & P. sergenti & \\
\hline & L. infantum & ZVL & Unknown & Dog \\
\hline Uganda & L. donovani & VL & P. martini & \\
\hline \multirow[t]{2}{*}{ Ukraine } & L. infantum & ZVL & $\begin{array}{l}P . \text { neglectus, } P \text {. } \\
\text { longiductus }\end{array}$ & Dog \\
\hline & L. donovani & VL & Unknown & \\
\hline \multirow[t]{3}{*}{ Uzbekistan } & L. infantum & ZVL & P. longiductus & Dog \\
\hline & L. major & $\mathrm{CL}$ & P. papatasi & R. opimus \\
\hline & L. tropica & $\mathrm{ACL}$ & P. sergenti & Human \\
\hline \multirow[t]{4}{*}{ Yemen } & L. infantum & ZVL & Unknown & Dog \\
\hline & L. donovani & $\mathrm{VL}$ & P. orientalis & \\
\hline & L. tropica & $\mathrm{CL}$ & P. sergenti & \\
\hline & L. major & ZCL & $\begin{array}{l}P \text {. bergeroti, } P \text {. } \\
\text { duboscqi, } P \text {. } \\
\text { papatasi }\end{array}$ & Unknown \\
\hline Zambia & Unknown & VL & Unknown & \\
\hline
\end{tabular}

Proven vector. anthropophilic and bites animal reservoir; found naturally infected with a parasite indistinguishable from that found in humans and reservoirs

Proven reservoir. harbours parasite in nature and has been shown by ecological studies to maintain the parasite population

Suspected vector (no asterisk): anthropophilic with geographical distribution compatible with that of endemic foci; may be suspected on the basis of epidemiological evidence; may have been found naturally infected, but parasite not identified; or suspected because it is a proven vector elsewhere

Suspected reservoir (no asterisk); found infected in nature, but the extent of infection not determined

ACL, anthroponotic cutaneous leishmaniasis; ZCL, zoonotic cutaneous leishmaniasis; VL, visceral leishmaniasis; ZVL, zoonotic visceral leishmaniasis; CL, cutaneous leishmaniasis; PKDL, post-kalaazar dermal leishmaniasis; DCL, diffuse cutaneous leishmaniasis; ML, mucosal leishmaniasis; AVL, anthroponotic visceral leishmaniasis 
Table 5.

Distribution of New World leishmaniasis by country or territory, 2009

\begin{tabular}{|c|c|c|c|c|}
\hline $\begin{array}{l}\text { Country or } \\
\text { territory }\end{array}$ & Leishmania spp. & Clinical form & $\begin{array}{l}\text { Proven or suspected } \\
\text { Lutzomyia vector }\end{array}$ & $\begin{array}{l}\text { Proven or } \\
\text { suspected animal } \\
\text { reservoir }\end{array}$ \\
\hline \multirow[t]{4}{*}{ Argentina } & L. guyanensis & ZCL & Unknown & Unknown \\
\hline & L. amazonensis & ZCL & Unknown & Unknown \\
\hline & L. braziliensis & ZCL, MCL & $\begin{array}{l}\text { Lu. whitmani, Lu. } \\
\text { neivai, Lu. migonei }\end{array}$ & Dog \\
\hline & L. infantum & ZVL & Lu. longipalpis & Dog \\
\hline \multirow[t]{2}{*}{ Belize } & L. braziliensis & ZCL & Lu. ovallesi & Unknown \\
\hline & L. mexicana & ZCL & Lu. olmeca olmeca & $\begin{array}{l}\text { Heteromys spp., } \\
\text { Nyctomys spp., } \\
\text { Ototylomys spp., } \\
\text { Sigmodon spp., } \\
\text { Oryzomys spp. }\end{array}$ \\
\hline
\end{tabular}

\begin{tabular}{|c|c|c|c|c|}
\hline \multirow[t]{5}{*}{ Bolivia } & L. braziliensis & ZCL, MCL & $\begin{array}{l}\text { Lu. nuneztovari } \\
\text { anglesi, Lu. carrerai } \\
\text { carrerai, Lu. } \\
\text { Ilanosmartinsi, Lu. } \\
\text { shawi, Lu. ayrozai, } \\
\text { Lu. yucumensis }\end{array}$ & Unknown \\
\hline & L. amazonensis & ZCL, DCL & Lu. flaviscutellata & Oryzomys spp. \\
\hline & L. infantum & ZVL & Lu. longipalpis & Dog \\
\hline & L. guyanensis & $\mathrm{ZCL}$ & Lu. shawi & $\begin{array}{l}\text { Choloepus spp., } \\
\text { Didelphis spp., } \\
\text { Tamandua spp. }\end{array}$ \\
\hline & L. lainsoni & ZCL & $\begin{array}{l}\text { Lu. nuneztovari } \\
\text { anglesi }\end{array}$ & Agouti paca \\
\hline \multirow[t]{2}{*}{ Brazil } & L. guyanensis & ZCL & $\begin{array}{l}\text { Lu. umbratilis, Lu. } \\
\text { anduzei, Lu. } \\
\text { whitmani }\end{array}$ & $\begin{array}{l}\text { Choloepus spp., } \\
\text { Tamandua spp., } \\
\text { Didelphis spp., } \\
\text { Proechimys spp. }\end{array}$ \\
\hline & L. amazonensis & $\mathrm{CL}$ & $\begin{array}{l}\text { Lu. flaviscutellata, } \\
\text { Lu. longipalpis }\end{array}$ & $\begin{array}{l}\text { Proechimys spp., } \\
\text { Oryzomys spp., } \\
\text { Wiedomys spp. }\end{array}$ \\
\hline
\end{tabular}



L. braziliensis $\quad \mathrm{ZCL}, \mathrm{MCL} \quad L u$. whitmani, Lu. Dog, Rattus rattus, intermedia, Lu. Akodon wellcomei, Lu. arviculoides, complexa, Lu. neivai, Bolomys spp., Lu. edwardsi, Lu. Nectomis spp., migonei Thrichomys spp.

L. infantum ZVL Lu. longipalpis, Lu. Dog, Lycalopex cruzi, Lu. almerio, Lu. vetulus, Cedocyon salesi thous, Didelphis albiventris, Cat

L. lainsoni ZCL Lu. ubiquitalis Agouti paca

$\begin{array}{llll}\text { L. shawi ZCL } \quad \text { Lu. whitmani } & \text { Cebus apella, }\end{array}$ Chiropotes satanus, Nasua nasua, Bradypus tridactylus, Choloepus didactylus

L. naiffi

ZCL

Lu. squamiventris,

Dasypus

$L u$. paraensis, Lu. novemcinctus amazonensis, Lu.ayrozai

L. lindenbergi ZCL Unknown Unknown

\begin{tabular}{|c|c|c|c|c|}
\hline \multirow[t]{2}{*}{ Colombia } & L. braziliensis & $\mathrm{ZCL}, \mathrm{MCL}$ & $\begin{array}{l}\text { Lu. spinicrassa, Lu. } \\
\text { colombiana, Lu. pia, } \\
\text { Lu. towsendi }\end{array}$ & $\begin{array}{l}\text { Dog, Akodon spp., } \\
\text { Micoureus } \\
\text { demerarae, } \\
\text { Melanomys } \\
\text { caliginosus, Rattus } \\
\text { rattus, Didelphis } \\
\text { marsupialis }\end{array}$ \\
\hline & L. panamensis & ZCL, MCL & $\begin{array}{l}\text { Lu. trapidoi, Lu. } \\
\text { gomezi, Lu. } \\
\text { panamensis, Lu. } \\
\text { yuilli }\end{array}$ & $\begin{array}{l}\text { Dog, Choloepus } \\
\text { hoffmani, } \\
\text { Metachirus } \\
\text { nudicaudatus, } \\
\text { Didelphis } \\
\text { marsupialis, } \\
\text { Coendou spp. }\end{array}$ \\
\hline
\end{tabular}




\begin{tabular}{|c|c|c|c|c|}
\hline & L. guyanensis & ZCL, MCL & $\begin{array}{l}\text { Lu. umbratilis, Lu. } \\
\text { longiflocosa }\end{array}$ & Unknown \\
\hline & L. colombiensis & $\mathrm{ZCL}$ & Lu. hartmanni & Unknown \\
\hline & L. amazonensis & ZCL, DCL & Lu. flaviscutellata & Unknown \\
\hline & L. mexicana & $\mathrm{ZCL}$ & Lu. columbiana & $\begin{array}{l}\text { Didelphis } \\
\text { marsupialis }\end{array}$ \\
\hline & L. infantum & ZVL & $\begin{array}{l}\text { Lu. longipalpis, Lu. } \\
\text { evansi }\end{array}$ & $\begin{array}{l}\text { Dog, Didelphis } \\
\text { marsupialis }\end{array}$ \\
\hline \multirow[t]{5}{*}{ Costa Rica } & L. panamensis & ZCL, MCL & $\begin{array}{l}\text { Lu. ylephiletor, Lu. } \\
\text { trapidoi }\end{array}$ & $\begin{array}{l}\text { Bradypus griseus, } \\
\text { Choloepus } \\
\text { hoffmanni, } \\
\text { Heteromys } \\
\text { desmarestianus }\end{array}$ \\
\hline & L. mexicana & $\begin{array}{l}\text { ZCL, MCL, } \\
\text { DCL }\end{array}$ & $\begin{array}{l}\text { Lu. olmeca olmeca, } \\
\text { Lu. olmeca bicolor }\end{array}$ & Unknown \\
\hline & L. braziliensis & ZCL, MCL & Lu. youngi & Unknown \\
\hline & L. garnhami & ZCL & Lu. youngi & Unknown \\
\hline & L. infantum & ZCL & $\begin{array}{l}\text { Lu. longipalpis, Lu. } \\
\text { evansi }\end{array}$ & Dog \\
\hline $\begin{array}{l}\text { Dominican } \\
\text { Republic }\end{array}$ & Unknown & $\mathrm{DCL}$ & Unknown & Unknown \\
\hline \multirow[t]{5}{*}{ Ecuador } & L. braziliensis & ZCL, MCL & Unknown & Unknown \\
\hline & L. panamensis & ZCL & $\begin{array}{l}\text { Lu. trapidoi, Lu. } \\
\text { hartmanni, Lu. } \\
\text { gomezi }\end{array}$ & $\begin{array}{l}\text { Potus flavus, } \\
\text { Tamandua } \\
\text { tetradactyla, } \\
\text { Sciurus vulgaris, } \\
\text { Choloepus } \\
\text { didactylus }\end{array}$ \\
\hline & L. guyanensis & ZCL & Unknown & Unknown \\
\hline & L. amazonensis & ZCL, DCL & Lu. flaviscutellata & Sciurus spp. \\
\hline & L. mexicana & ZCL, DCL & Lu. ayacuchensis & Unknown \\
\hline El Salvador & L. infantum & ZVL, CL & Lu. longipalpis & Dog \\
\hline
\end{tabular}




\begin{tabular}{|c|c|c|c|c|}
\hline \multirow[t]{5}{*}{$\begin{array}{l}\text { French } \\
\text { Guiana }\end{array}$} & L. guyanensis & ZCL & Lu. umbratilis & $\begin{array}{l}\text { Choleopus } \\
\text { didactylus, } \\
\text { Proechimys spp., } \\
\text { Didelphis } \\
\text { marsupialis }\end{array}$ \\
\hline & L. braziliensis & ZCL, MCL & $\begin{array}{l}\text { Lu. wellcomei, Lu. } \\
\text { intermedia }\end{array}$ & Unknown \\
\hline & L. amazonensis & ZCL & Lu flaviscutellata & Proechimys spp. \\
\hline & L. naiffi & ZCL & Unknown & Unknown \\
\hline & L. lainsoni & ZCL & Unknown & Unknown \\
\hline \multirow[t]{4}{*}{ Guatemala } & L. infantum & ZVL & Lu. longipalpis & Dog \\
\hline & L. panamensis, & ZCL, MCL & $\begin{array}{l}\text { Lu. ylephiletor, Lu. } \\
\text { panamensis, Lu. } \\
\text { trapidoi }\end{array}$ & Unknown \\
\hline & L. braziliensis & ZCL, MCL & $\begin{array}{l}\text { Lu. ovallesi, Lu. } \\
\text { panamensis, Lu. } \\
\text { ylephiletor }\end{array}$ & Rattus rattus \\
\hline & L. mexicana & ZCL, DCL & Lu. olmeca olmeca & Unknown \\
\hline Guyana & L. guyanensis & ZCL & $\begin{array}{l}\text { Lu. umbratilis, Lu. } \\
\text { anduzei }\end{array}$ & Unknown \\
\hline
\end{tabular}

\begin{tabular}{|c|c|c|c|c|}
\hline \multirow[t]{3}{*}{ Honduras } & L. infantum & ZVL, CL & Lu. longipalpis & Dog \\
\hline & L. panamensis & ZCL, MCL & $\begin{array}{l}\text { Lu. ylephiletor, Lu. } \\
\text { panamensis, Lu. } \\
\text { trapidoi }\end{array}$ & Unknown \\
\hline & L. braziliensis & ZCL, MCL & $\begin{array}{l}\text { Lu. ovallesi, Lu. } \\
\text { panamensis, Lu. } \\
\text { ylephiletor }\end{array}$ & Unknown \\
\hline \multirow[t]{3}{*}{ Mexico } & L. braziliensis & ZCL, MCL & $\begin{array}{l}\text { Lu. ovallesi, Lu. } \\
\text { cruciata }\end{array}$ & Unknown \\
\hline & L. mexicana & $\begin{array}{l}\mathrm{ZCL}, \mathrm{MCL}, \\
\mathrm{DCL}\end{array}$ & $\begin{array}{l}\text { Lu. olmeca olmeca, } \\
\text { Lu. cruciata, Lu. } \\
\text { shannoni }\end{array}$ & $\begin{array}{l}\text { Heteromys spp., } \\
\text { Nyctomys spp., } \\
\text { Ototylomys spp., } \\
\text { Sigmodon spp., } \\
\text { Peromyscus spp. }\end{array}$ \\
\hline & L. infantum & ZVL & $\begin{array}{l}\text { Lu. longipalpis, Lu. } \\
\text { evansi }\end{array}$ & Dog \\
\hline
\end{tabular}




\begin{tabular}{|c|c|c|c|c|}
\hline \multirow[t]{3}{*}{ Nicaragua } & L. infantum & ZVL, CL & $\begin{array}{l}\text { Lu. longipalpis, Lu. } \\
\text { evansi }\end{array}$ & Dog \\
\hline & L. panamensis & $\mathrm{ZCL}$ & $\begin{array}{l}\text { Lu. trapidoi, Lu. } \\
\text { ylephiletor, Lu. } \\
\text { cruciata, Lu. } \\
\text { panamensis }\end{array}$ & Unknown \\
\hline & L. braziliensis & ZCL, MCL & Lu. panamensis & Unknown \\
\hline \multirow[t]{3}{*}{ Panama } & L. panamensis & ZCL, MCL & $\begin{array}{l}\text { Lu. trapidoi, Lu. } \\
\text { ylephiletor, Lu. } \\
\text { sanguinaria, Lu. } \\
\text { panamensis, Lu. } \\
\text { gomezi }\end{array}$ & $\begin{array}{l}\text { Choloepus } \\
\text { hoffmanni }\end{array}$ \\
\hline & L. braziliensis & ZCL & Lu. panamensis & Unknown \\
\hline & L. colombiensis & ZCL & Unknown & $\begin{array}{l}\text { Choloepus } \\
\text { hoffmanni }\end{array}$ \\
\hline \multirow[t]{2}{*}{ Paraguay } & L. braziliensis & ZCL, MCL & $\begin{array}{l}\text { Lu. whitmani, Lu. } \\
\text { migonei, Lu. } \\
\text { Intermedia }\end{array}$ & Unknown \\
\hline & L. infantum & ZVL & Lu. longipalpis & Dog \\
\hline \multirow[t]{5}{*}{ Peru } & L. peruviana & ZCL, MCL & $\begin{array}{l}\text { Lu. peruensis, Lu. } \\
\text { verrucarum, Lu. } \\
\text { ayacuchensis }\end{array}$ & $\begin{array}{l}\text { Dog, Didelphis } \\
\text { albiventris, } \\
\text { Phyllotis andinum, } \\
\text { Akodon spp. }\end{array}$ \\
\hline & L. lainsoni & ZCL & Lu. ubiquitalis & Unknown \\
\hline & L. amazonensis & ZCL & Unknown & Unknown \\
\hline & L. guyanensis & ZCL, MCL & Unknown & Unknown \\
\hline & L. braziliensis & $\begin{array}{l}\text { ZCL, MCL, } \\
\text { DCL }\end{array}$ & $\begin{array}{l}\text { Lu. tejadai, Lu. } \\
\text { pescei }\end{array}$ & Unknown \\
\hline \multirow[t]{3}{*}{ Suriname } & L. guyanensis & ZCL & $\begin{array}{l}\text { Lu. umbratilis, Lu. } \\
\text { anduzei }\end{array}$ & Unknown \\
\hline & L. amazonensis & ZCL & Lu. flaviscutellata & Unknown \\
\hline & L. lainsoni & ZCL & Unknown & Unknown \\
\hline $\begin{array}{l}\text { United States } \\
\text { of America }\end{array}$ & L. mexicana & ZCL, DCL & $\begin{array}{l}\text { Lu. anthophora, Lu. } \\
\text { diabolica }\end{array}$ & Neotoma spp. \\
\hline
\end{tabular}




\begin{tabular}{|c|c|c|c|c|}
\hline & L. infantum & Unknown & Unknown & Dog \\
\hline \multirow[t]{8}{*}{ Venezuela } & L. braziliensis & ZCL, MCL & $\begin{array}{l}\text { Lu. ovallesi, Lu. } \\
\text { trinidanensis, Lu. } \\
\text { spinicrassa, Lu. } \\
\text { panamensis }\end{array}$ & Unknown \\
\hline & L. colombiensis & ZCL & $\begin{array}{l}\text { Lu. panamensis, Lu. } \\
\text { gomezi }\end{array}$ & Unknown \\
\hline & L. venezuelensis & ZCL, DCL & Lu. olmeca bicolor & Unknown \\
\hline & L. amazonensis & ZCL, DCL & $\begin{array}{l}\text { Lu. flaviscutellata, } \\
\text { Lu. reducta }\end{array}$ & Unknown \\
\hline & L. pifanoi & DCL & Lu. flaviscutellata & Unknown \\
\hline & L. garnhami & ZCL & Lu. youngi & Unknown \\
\hline & L. infantum & ZVL & $\begin{array}{l}\text { Lu. longipalpis, Lu. } \\
\text { evansi, Lu. } \\
\text { pseudolongipalpis }\end{array}$ & Dog \\
\hline & L. guyanensis & ZCL & Unknown & Unknown \\
\hline
\end{tabular}

Proven vector. anthropophilic and bites animal reservoir; found naturally infected with a parasite indistinguishable from that found in humans and reservoirs

Proven reservoir. harbours parasite in nature and has been shown by ecological studies to maintain the parasite population

Suspected vector (no asterisk): anthropophilic with geographical distribution compatible with that of endemic foci; may be suspected on the basis of epidemiological evidence; may have been found naturally infected, but parasite not identified; or suspected because it is a proven vector elsewhere

Suspected reservoir (no asterisk); found infected in nature, but the extent of infection not determined $\mathrm{ZCL}$, zoonotic cutaneous leishmaniasis; $\mathrm{MCL}$, mucocutaneous leishmaniasis; DCL, diffuse cutaneous leishmaniasis; CL, cutaneous leishmaniasis; ZVL, zoonotic visceral leishmaniasis

\subsection{Estimated burden}

Leishmaniasis is endemic in 98 countries or territories, with more than 350 million people at risk. Published figures indicate an estimated incidence of 2 million new cases per year $(0.5$ million of visceral leishmaniasis and 1.5 million of cutaneous leishmaniasis). Visceral leishmaniasis causes an estimated over 50000 deaths annually, a rate surpassed among parasitic diseases only by malaria, and 2357000 disability-adjusted life years lost, placing leishmaniasis ninth in a global analysis of infectious diseases. These figures are under review. Making accurate estimates is extremely challenging, however, because (as for other neglected tropical diseases) empirical data 
on leishmaniasis incidence are sparse. Estimates of disability-adjusted life years are likely to be underestimates of the burden, because the weights used fail to account for secondary effects, such as stigma (for cutaneous leishmaniasis and PKDL) and the economic effect on households of both cutaneous and visceral leishmaniasis and their treatment (see section 3.7).

Coinfection with HIV intensifies the burden of visceral and cutaneous leishmaniasis by causing severe forms that are more difficult to manage (see section 3.2.4). As of March 2010, Leishmania-HIV coinfection had been reported in 35 endemic countries. According to a WHO coordinated monitoring system involving 28 institutions worldwide, the number of new cases has declined in Europe since the end of the 1990s, mainly due to access to highly active antiretroviral therapy. In other parts of the world, however, where there is limited access to such treatment, the prevalence is steadily rising, especially in northern Ethiopia, where the rate of HIV infection increased from 19\% of people with visceral leishmaniasis in 1998-1999 to 34\% in 2006-2007. In Brazil, India, Nepal and the Sudan, the estimated prevalence has so far remained below $10 \%$ but is expected to rise, as long as there continues to be little access to antiretroviral treatment. As the HIV pandemic in the tropics is expanding into rural and remote areas endemic for visceral leishmaniasis, HIV-visceral leishmaniasis coinfection is a rapidly expanding problem, increasing the urgency of a comprehensive approach for control of visceral leishmaniasis.

The characteristic geographical clustering of leishmaniasis cases, especially when the transmission is anthroponotic, complicates estimation of disease burden, because the incidence figures for a small area cannot be extrapolated confidently to a much larger area. Passive surveillance data from health facilities are generally the most comprehensive source available for estimating country-level disease burden; however, the extent of underreporting in most leishmaniasis-endemic countries is known to be substantial, ranging from two- to 40-fold in targeted studies. These findings imply that different, largely undetermined correction factors should be applied for each setting. Underreporting of deaths is even more pronounced. The results of a village-based study in India suggested that as many as $20 \%$ of visceral leishmaniasis patients, disproportionately poor and female, died before their disease was recognized.

The leishmaniases are widely dispersed, with transmission to humans on five continents, but the human disease burden is concentrated mainly in a few major foci. Anthroponotic $L$. donovani transmission in a relatively small but heavily populated area spanning northeastern India, southeastern Nepal and central Bangladesh accounts for more than two thirds of all cases of visceral leishmaniasis in the world. The East African L. donovani focus, also with an 
important component of anthroponotic transmission, is the second largest focus of visceral leishmaniasis, with the highest incidence in Ethiopia and the Sudan. The other two important foci, both caused by zoonotic L. infantum transmission, are the Mediterranean Basin, the Middle East and western Asia; and the New World, predominantly in Brazil. It has been estimated that more than $90 \%$ of the burden of visceral leishmaniasis is concentrated in Bangladesh, Brazil, Ethiopia, India, Nepal and the Sudan.

Cutaneous leishmaniasis is even more widely dispersed, with major foci of anthroponotic L. tropica transmission stretching from India through Central and western Asia into northern Africa, zoonotic transmission of $L$. major from Central Asia through western Asia into northern Africa and L. aethiopica in East Africa. The other major area of transmission is made up of many ecologically different foci with various zoonotic Leishmania species and affects countries throughout the Americas, from Argentina to the southern USA. Up to $90 \%$ of cases of cutaneous leishmaniasis occur in Afghanistan, Algeria, the Islamic Republic of Iran, Saudi Arabia and the Syrian Arab Republic and in Bolivia, Brazil, Colombia, Nicaragua and Peru.

The distribution of leishmaniasis is dynamic: Colombia, Nicaragua and Pakistan recently reported large increases in the incidence of cutaneous leishmaniasis, while Ethiopia and the Sudan have experienced epidemics of the visceral form in previously unaffected areas. Many endemic areas show large fluctuations in incidence over time, which are sometimes attributable to specific events, such as population displacement or climate factors. Climatic, socioeconomic and other environmental changes could expand the geographical range of the vectors and leishmaniasis transmission in the future (see section 2.6.6). 


\section{Control strategies by nosogeographical entity}

\subsection{Visceral leishmaniasis caused by $L$. donovani and $L$. infantum (L. chagasi)}

\subsubsection{Visceral leishmaniasis caused by L. donovani on the Indian subcontinent}

Geographical distribution (see Table 4 and Figure 3)

Bangladesh, Bhutan, India (Bihar, Jharkhand, West Bengal, Uttar Pradesh and recently Assam) and Nepal. A few cases were reported recently in Sri Lanka.

\section{Epidemiological features}

Serological testing of populations has revealed large numbers of cryptic and oligosymptomatic infections. All the available evidence indicates that the disease is transmitted from human to human by an anthropophilic and zoophilic sandfly, $P$. argentipes. It is usually endemic (in India, in 2008, 33 233 cases were officially reported, of which 28125 were in Bihar State, 3690 in Jharkhand and 1256 in West Bengal). When account is taken of underreporting, the actual number of cases is estimated to be five to eight times higher. Severe epidemics can occur; e.g. in 1977-1987, 185000 new patients were recorded in Bihar State. PKDL can occur as a sequel: $1-10 \%$ of patients with visceral leishmaniasis develop PKDL; however, this form can appear in the absence of any previous episode of visceral leishmaniasis. In 2005, the ministers of health of Bangladesh, India and Nepal signed a memorandum of understanding to eliminate kala-azar by 2015 , with a target annual incidence of less than 1/10 000 at district level.

\section{Minimal operations}

Passive case detection and complete treatment will reduce the public health problem, while effective case reporting is essential for monitoring progress. The mainstay in the diagnosis of kala-azar is serological testing based on the rapid dipstick rK39 antigen test to confirm clinically suspected cases (fever for more than 2 weeks and splenomegaly in the absence of malaria). As it can 
Figure 3.

Geographical distribution of visceral leishmaniasis in the Old and New world

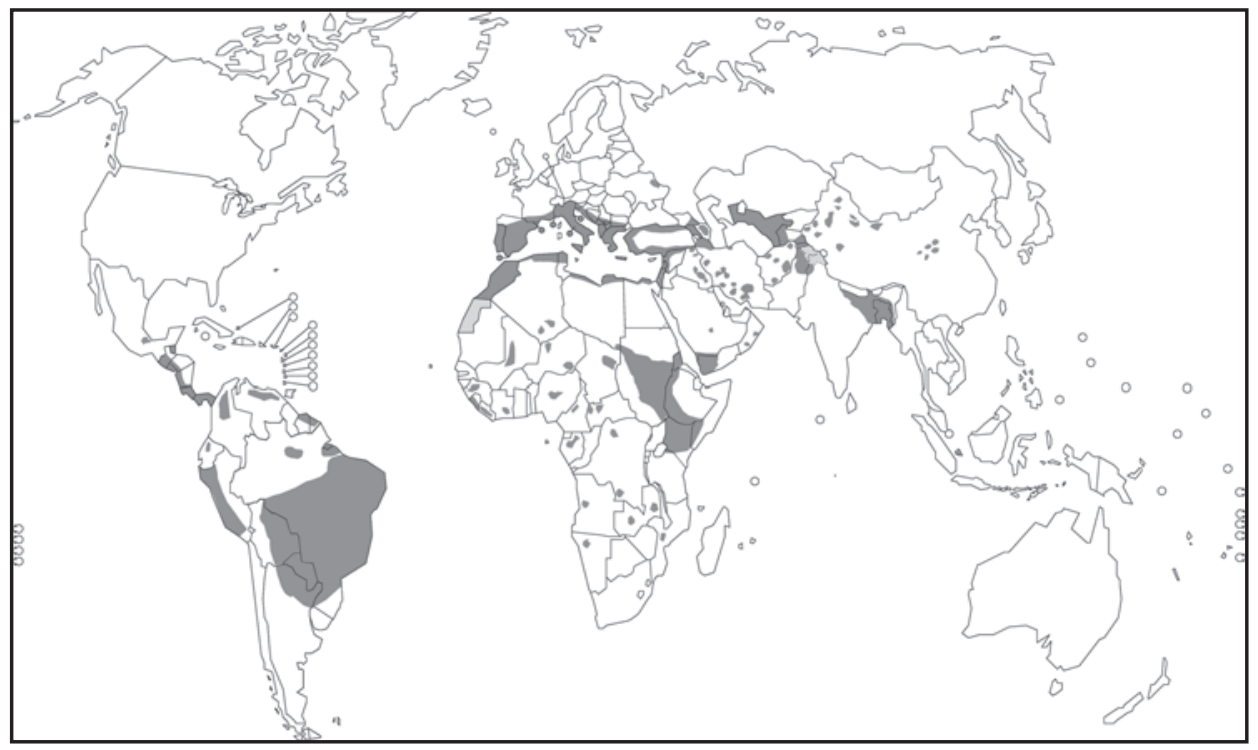

be performed in any setting by a trained health worker, there is no need for parasitological confirmatory tests in all cases. Suspected cases with negative serological results should be referred to facilities where appropriate laboratory diagnostic testing is available.

\section{Reservoir control}

Active case detection and treatment of patients should reduce the transmission rate. As cases of PKDL are considered to constitute an important residual reservoir, their detection and treatment are important, especially at times of low prevalence. PKDL patients often do not seek treatment or may default from treatment. Strengthening passive and active surveillance of PKDL is a priority.

\section{Vector control}

Phlebotomus argentipes is strictly peridomestic and susceptible to residual insecticide spraying in most areas. In epidemics, large-scale indoor residual spraying should be used, covering all buildings, including houses and animal shelters, in affected and surrounding areas. In endemic conditions, spraying may be localized in infected villages and should be timed to precede maximum sandfly density. The effectiveness of insecticide-treated nets and longlasting nets is still under evaluation. Environmental measures, such as sanitation in peridomestic areas and housing improvement, are important 
complementary measures, as they contribute to reducing sandfly breeding sites.

\section{Evaluation}

All control activities should be accompanied by adequate surveillance and reporting, so as to provide some indication of the effect. Susceptibility to insecticides and sandfly vector density should be monitored periodically.

\subsubsection{Visceral leishmaniasis in East Africa and the south-west Arabian peninsula caused by $\mathrm{L}$. donovani and $\mathrm{L}$. infantum}

\section{Geographical distribution (see Table 4 and Figure 3)}

Eritrea, Ethiopia (Metema-Humera in the northwestern lowlands; Libo Kemkem and Fogera districts in Amhara regional State and north of Lake Turkana; in the south, the Segen and Woito valleys, the Genale and Gelana river basins and west Moyale at the border with Kenya), Djibouti, Kenya (Machacos, Kitui, West Pokot, Masinga, Meru, Baringo, Turkana), Saudi Arabia (south-west region: Jazan, Assir), Somalia, the Sudan (North: Gadaref, Blue Nile, White Nile, Sinnar, South Kordofan and West Darfur states; South: Upper Nile, Jonglei, Unity States, Eastern Equatoria), Uganda (northeastern focus: Pokot Department) and Yemen (Taiz, Lahj and Ibb governorates)

\section{Epidemiological features}

While foci of anthroponotic transmission are predominant and are related to L. donovani, foci of zoonotic transmission with dogs as the domestic reservoir have also been identified. They have been provisionally identified related to L. infantum by isoenzyme electrophoresis.

The proven vectors, $P$. martini and $P$. orientalis, are not synanthropic; the former is sometimes closely associated with termite hills and the latter with specific Acacia and Balanites woodlands. Outbreaks have been associated with the massive movement of nonimmune people to endemic areas, malnutrition, civil unrest and associated diseases. Serological testing of populations has indicated large numbers of asymptomatic infections.

\section{Minimal operations}

Passive case detection and treatment will reduce morbidity and mortality, but it is not known whether they will reduce transmission. Clinically suspected cases of visceral leishmaniasis should be confirmed by serological testing (rK39). Passive case detection leaves many cases undiagnosed and untreated and is often insufficient in epidemics. 
Reservoir control

Active detection of cases and treatment may reduce transmission. PKDL is more frequent (up to 50\% reported in Sudan) but less severe (most cases cure spontaneously) than in Asia.

\section{Vector control}

While spraying of termite hills has been used in an attempt to control $P$. martini, this measure has not been adequately evaluated. Use of insecticidetreated nets in eastern Sudan was associated with reduced disease incidence in a retrospective analysis. Prospective evaluation of the effectiveness of insecticide-treated nets and long-lasting insecticidal nets in this area is highly recommended. In epidemics, spraying of houses in densely populated areas with insecticide appears to be a logical approach but has not been fully evaluated.

\section{Evaluation}

Annual incidence should be assessed by active detection of cases and serological surveys.

\subsubsection{Visceral leishmaniasis caused by L. donovani in other places}

Geographical distribution (Table 4 and Figure 3)

China, Cyprus and Iraq

\section{Epidemiological features}

Only sporadic cases occur.

\section{Minimal operations}

Medical authorities must have the facilities and knowledge to diagnose and treat patients. Late diagnosis should be monitored as an indicator of the performance of the control programme.

\subsubsection{Foci of visceral leishmaniasis caused by L. infantum with known or assumed canine reservoir hosts}

Geographical distribution (see Table 4 and Figure 3)

Visceral leishmaniasis with known or assumed canine reservoirs: Afghanistan, Albania, Algeria, Argentina, Armenia, Azerbaijan, Bolivia, Bosnia and Herzegovina, Brazil, Bulgaria, Chad, Central African Republic, China, Colombia, Croatia, Cyprus, Egypt, El Salvador, France, Gambia, Georgia, Greece, Guatemala, Honduras, Islamic Republic of Iran, Iraq, Israel, Italy, Jordan, Kazakhstan, Kyrgyzstan, Lebanon, Libyan Arab Jamahiriya, 
Macedonia, Malta, Mauritania, Mexico, Monaco, Montenegro, Morocco,

Nicaragua, Oman, Pakistan, Paraguay, Portugal, Romania, Saudi Arabia, Senegal, Slovenia, Spain, Syrian Arab Republic, Tunisia, Turkey, Turkmenistan, Ukraine, Uzbekistan and Venezuela

\section{Epidemiological features}

The disease is zoonotic; domestic dogs and wild canines (foxes, jackals, wolves) serve as reservoir hosts, bringing the infection close to humans. There is a range of known or suspected vectors in different foci, only some of which enter houses. Transmission can occur by syringe sharing in HIV immunosuppressed patients. The incidence of human disease is generally low. The main well-known endemic foci are:

- the Mediterranean littoral and Portugal: domestic dogs are the main reservoir host, and the European fox, Vulpes vulpes, has been found to be infected. The vectors are sandflies of the subgenera Larroussius and Adlerius (see Table 4), which bite and rest both indoors and outdoors. The disease is found in children and adults in varying proportions, and there is strong evidence of frequent subclinical infection of long duration but unknown morbidity.

- Central Asia: Active foci are found in Afghanistan, Armenia, Azerbaijan, Georgia, Kazakhstan, Kyrgyzstan, Turkmenistan and Uzbekistan. The reservoir hosts are domestic dogs, foxes Vulpes spp. and jackals. The vectors are thought to be $P$. kandelakii, $P$. balcanicus, $P$. longiductus and P. smirnovi.

- Saudi Arabia: Several foci are known along the foothills of the Asir Mountains. There is evidence that domestic dogs harbour $L$. infantum, while $L$. donovani and $L$. infantum are found in humans. P. orientalis is the proven vector for $L$. donovani. The disease is notifiable in Saudi Arabia, and 30-40 cases are recorded annually.

- Islamic Republic of lran: The main foci are in East Azerbaijan, Fars and Khuzestan. The vector is believed to be P. kandelakii. Dogs are the domestic reservoirs, and foxes and jackals have been found to be infected.

- Iraq: There are stable foci of $L$. donovani and L. infantum in central Iraq between the Tigris and the Euphrates rivers and in the Greater Baghdad area. Up to 1991, 3000 cases were reported annually; after 1996, a new focus appeared in southern Iraq; and in 2002, 840 cases of visceral leishmaniasis were reported in Thi Qar Governorate, mostly in Nassiriyah district, with an incidence of 5/10 000. Most of the cases were in children under 5 years of age. Dogs have been found to be infected, but serological surveys suggest that the jackal is the wild 
reservoir host. The vector has not yet been incriminated; $P$. alexandri is suspected in spite of its apparently low density.

- China: Foci of $L$. infantum and $L$. donovani are present, mainly in Gansu, Sichuan, Shanxi and Shaanxi provinces and the Xinjiang and Inner Mongolia autonomous regions. In 2008, 529 cases of visceral leishmaniasis were reported officially. Dogs are the main reservoir host and play an important role in the hilly and mountainous endemic areas of the northwest. Most cases are in children under 5 years of age. $P$. chinensis is the main vector. In the desert areas of the northwest (Xinjiang Autonomous Region, Turfan county), P. alexandri has been found to be naturally infected by $L$. infantum. In the alluvial plains of eastern China, dogs seem to play a minor role in transmission; the sandflies $P$. alexandri, $P$. wui and $P$. chinensis are proven vectors.

In the suburbs of Beijing, a raccoon-dog (Nyctereutes procyonoides) was reported to be infected by $L$. infantum. No other animal, among more than 2000 studied, was found to be infected. All the isolates from humans were $L$. infantum, except for one from Gansu identified as $L$. donovani.

- Latin America: Visceral leishmaniasis occurs in many foci in various countries (see Table 5). The available evidence suggests that the structure of these foci is similar. Sibling species of Lu. longipalpis are the main vectors in Latin America, and Lu. evansi is also a vector in Colombia and Venezuela. Several other species are suspected to be vectors (see Table 5). Two species of fox (Cerdocyon thous and Lycalopex vetulus) have been incriminated as wild reservoir hosts. In Colombia, Didelphis marsupialis has been found to be infected.

\section{Minimal operations}

Passive case detection, reporting and treatment are recommended in order to limit mortality and provide some indication of the prevalence. This action cannot, however, be expected to reduce the incidence, as the disease is zoonotic.

\section{Reservoir control}

Infection in dogs should be monitored, and the management of infected dogs should be treatment or elimination. Stray and feral dogs should be controlled whenever possible, although these actions may not eliminate human disease because of the presence of wild animal reservoirs. Use of topical insecticides on domestic dogs has reduced the incidence of canine and human visceral leishmaniasis. 


\section{Vector control}

In a number of foci, the incidence of visceral leishmaniasis has fallen after houses were sprayed with insecticides for the purposes of malaria control. If used to control leishmaniasis, spraying should be carried out immediately before the peak annual vector density and be maintained for several years. This control measure is appropriate only for endophilic species such as $P$. longiductus in central Asia and Lu. longipalpis and Lu. evansi in Latin America.

\section{Evaluation}

The effect of all control measures can be assessed by changes in the annual incidence of visceral leishmaniasis in a population, as determined by passive case detection or, preferably, by active detection accompanied by regular serological surveys of populations at risk, with special attention to children of preschool age.

\subsection{Anthroponotic cutaneous leishmaniasis caused by L. tropica}

\section{Geographical distribution (see Table 4 and Figure 4)}

Afghanistan (especially Kabul, Herat and Kandahar and neighbouring villages), Azerbaijan, India, the Islamic Republic of Iran, Iraq, Israel, Morocco, Pakistan, the Syrian Arab Republic, Turkey and Uzbekistan

\section{Epidemiological features}

The disease is found predominantly in densely populated settlements, where person-to-person transmission is maintained by $P$. sergenti. Some foci survive indefinitely (independent foci), while others (small settlements) seem to depend on repeated introductions (dependent foci). In dependent foci, where the disease is not always present, people of all ages may be affected, which increases its public health importance. Leishmaniasis recidivans, the lupoid or tuberculoid, chronic form (as an occasional sequel of anthroponotic cutaneous leishmaniasis), may last for many years, and patients may therefore carry parasites throughout the long periods of low transmission.

\section{Minimal operations}

Passive detection serves mainly to identify severe cases; the extent to which treatment of these cases will reduce transmission depends on the proportion they represent of all cases found. Treatment of patients with leishmaniasis recidivans is particularly valuable at times of low transmission. Improved housing and environmental sanitation are beneficial in reducing breeding sites. 
Anthroponotic cutaneous leishmaniasis has been almost eradicated in Central Asia, and its incidence was greatly reduced in many foci by antimalarial measures. This is a disease against which control measures can be very effective. Commumity participation is desirable and particularly appropriate.

\section{Reservoir control}

Active detection of cases, especially among children and migratory people, will reduce transmission. Cases of leishmaniasis recidivans should be sought particularly in periods of low transmission.

\section{Vector control}

The recommended measures include spraying of residual insecticide inside and outside houses where human cases of leishmaniasis have been found, as well as neighbouring houses, at the beginning of the active season for sandflies. Clearing of rubbish and construction materials from houses, streets and vacant land might have a long-term effect if maintained. Similarly, covering the lower parts of the walls of buildings with concrete, asphalting streets and covering courtyards with bricks, cement or other materials that do not permit the breeding of sandflies are effective. While $P$. sergenti is not known to be resistant to insecticides, its susceptibility should be checked regularly in any control scheme. Control will be achieved only by comprehensive coverage of independent foci. Insecticide-treated nets are useful in reducing incidence in foci of anthroponotic cutaneous leishmaniasis. Use of long- lasting insecticidal nets should be promoted.

Warning: Inadequate control measures, especially for anthroponotic cutaneous leishmaniasis, may not reduce the total number of cases but merely increase the mean age at which infection is contracted, thus increasing the severity of the disease. Any control programme involving reduction of transmission must be planned to be effective over the long term, and the budgetary requirements must be defined accordingly.

\section{Evaluation}

Prevalence rates in humans should be measured by active detection of cases. The mean age of people with lesions reflects incidence, and the ageprevalence distribution should be measured carefully in sample surveys. Monitoring of sandfly densities will provide a measure of the effectiveness of insecticide application.

\subsection{Sporadic cutaneous leishmaniasis caused by L. tropica and related species}

Geographical distribution (see Table 4 and Figure 4) 
Algeria, Egypt, Ethiopia, Greece, Israel, Jordan, Kenya, Libyan Arab Jamahiriya, Morocco, Namibia, Saudi Arabia, Tunisia and Yemen

Figure 4.

Geographical distribution of Old World cutaneous leishmaniasis due to $L$. tropica and related species and $L$. aethiopica

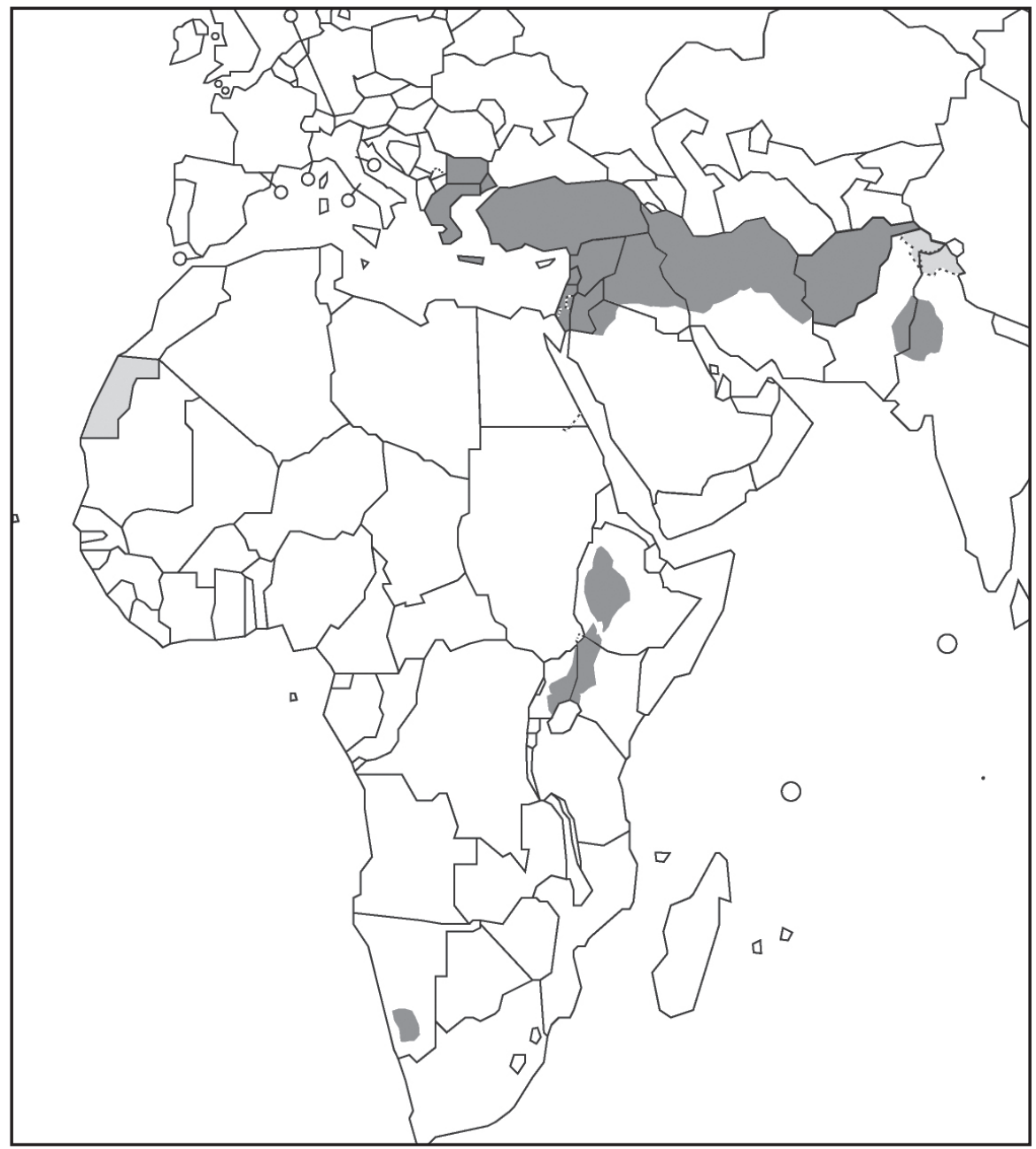

\section{Epidemiological features}

In foci of $L$. tropica with few or sporadic cases, the disease is known or suspected to be zoonotic. Hyraxes are among the suspected reservoir hosts. In Kenya, the vector is $P$. guggisbergi. Elsewhere, the vectors are not known with certainty. 


\section{Minimal measures}

Where practitioners and the required facilities are available, early diagnosis and treatment should be promoted.

\subsection{Zoonotic cutaneous leishmaniasis caused by L. major}

\section{Geographical distribution (see Table 4 and Figure 5)}

Afghanistan, Algeria, Azerbaijan, Burkina Faso, Cameroon, Chad, Egypt, Ethiopia, Gambia, Georgia, Ghana, Guinea, Guinea Bissau, India, the Islamic Republic of Iran, Iraq, Israel, Jordan, Kazakhstan, Kenya, Kuwait, the Libyan Arab Jamahiriya, Mali, Mauritania, Mongolia, Morocco, Niger, Nigeria, Oman, Pakistan, Saudi Arabia, Senegal, the Sudan, the Syrian Arab Republic, Tunisia, Turkey, Turkmenistan, Uzbekistan and Yemen

\section{Epidemiological features}

The main features are rurality and proneness to epidemics. On the basis of reservoir hosts and vector species, four main transmission systems can be identified: R. opimus (great gerbil) and P. papatasi; Psammomys spp. (fat sand rats) and $P$. papatasi; Meriones spp. (jirds) and $P$. papatasi or $P$. salehi; and Arvicanthis, Tatera or Mastomys spp. and P. duboscqi or $P$. papatasi.

\section{Minimal operations}

Passive detection of cases and recording are essential, with treatment. Spontaneous healing of lesions is generally associated with immunity to reinfection.

\section{Reservoir control}

Rodent control can be highly effective in reducing zoonotic cutaneous leishmaniasis

- In foci with Rhombomys as a reservoir host, spectacular results were achieved in the former USSR, with almost complete destruction of colonies by ploughing or poisoning and preventing colonization or reinvasion by artificial (irrigation canals) or natural barriers and by cultivation. Even small numbers of residual burrows that escape destruction can result in continued transmission.

- In foci with Psammomys as a reservoir host, successful control is difficult. Deep ploughing was used in Jordan, Saudi Arabia, Tunisia and the former USSR, but the method is expensive and its sustainability is low. In establishing new human settlements, it is important to choose sites distant from Psammomys colonies. Removal of Chenopodiaceae 
Figure 5.

Geographical distribution of Old World cutaneous leishmaniasis due to L. major

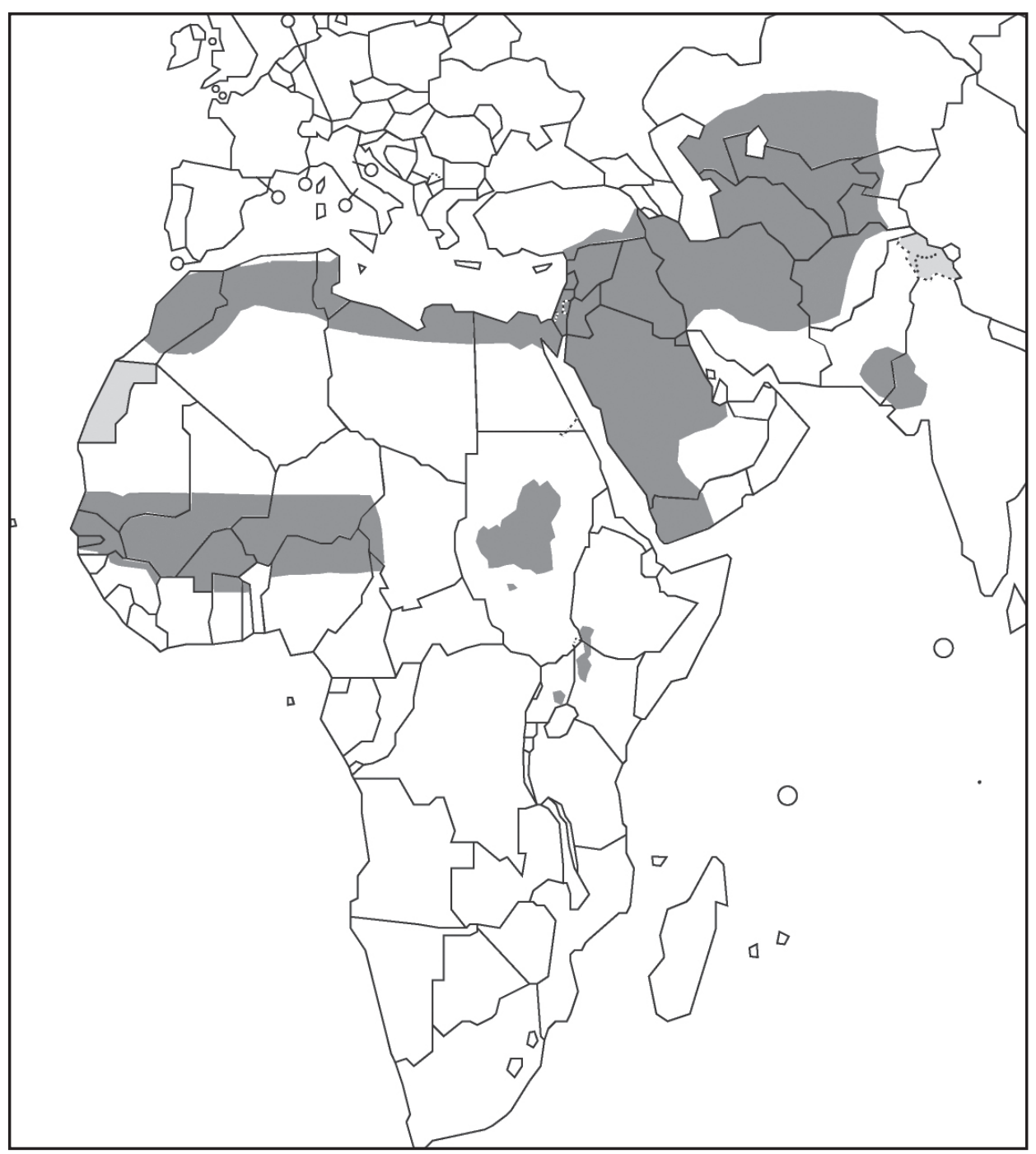

should reduce the population of Psammomys and decrease the risk for infection.

- In foci with Meriones as a reservoir host, poisoning of burrow entrances is possible; however, its effect on the transmission of leishmaniasis must be carefully evaluated. Poison can be accidentally absorbed by children and domestic animals such as goats and sheep. 


\section{Vector and reservoir control}

The destruction of gerbil burrows by deep ploughing in order to eliminate both the reservoir and the breeding and resting sites of $P$. papatasi was effective in certain parts of the former USSR and more recently in the Islamic Republic of Iran, Jordan, Saudi Arabia and Tunisia, but the method is expensive and its sustainability is low.

\section{Evaluation}

Assessments should be made continually by collecting and compiling the results of passive case detection. A more accurate evaluation is possible when passive surveillance is combined with spot surveys by active case detection in selected populations in areas of high priority. Another good indicator of transmission is sequential testing of preschool children with the leishmanin skin test before, during and after control measures. Assessment of rodent populations before and after the measures will provide information on the effectiveness of rodent control per se.

\subsection{Zoonotic cutaneous leishmaniasis in the Eastern African highlands caused by $L$. aethiopica}

\section{Geographical distribution (see Table 4 and Figure 4)}

Ethiopia, Kenya and Uganda

\section{Epidemiological features}

Stable foci of low endemicity are maintained by hyraxes (Procavia, Heterohyrax and Dendrohyrax spp.), and the parasite (L. aethiopica) is transmitted by $P$. longipes and $P$. pedifer. Human cases are closely associated with proximity to hyrax colonies. Lesions usually last a long time and heal spontaneously; however, in a small percentage of cases, diffuse cutaneous or mucocutaneous leishmaniasis develops.

\section{Minimal operations}

Passive and active case detection, treatment, reporting and data analysis should be performed.

\section{Reservoir control}

Small-scale eradication of hyraxes in the proximity of dwellings is effective.

\section{Vector control}

Vector control alone is unlikely to be effective; however, hyrax control should include fogging of their habitats to reduce the sandfly density. 


\section{Evaluation}

Active case detection and recording are essential for assessing control measures.

\subsection{Cutaneous leishmaniasis caused by L. peruviana}

Geographical distribution (see Table 5 and Figure 6)

Peru (high Andean valleys at 800-3000 m above sea level)

\section{Epidemiological features}

The natural reservoir hosts are probably wild marsupials and rodents; Didelphis albiventris, Phyllotis andinum and Akodon spp. have been found to be infected. Dogs are the principal peridomestic reservoir. Lutzomyia peruensis, $L u$. ayacuchensis and $L u$. verrucarum have been found to be naturally infected and are the most likely vectors. Transmission is seasonal: sandflies are absent from populated areas during the dry, cold season, although they can still be found in some rural areas in small villages and cultivated plots with rock terraces and fences. Because of the very high prevalence, the number of serious lesions is high, although they constitute a low proportion of all cases. Lesions occur predominantly in children below school age.

\section{Minimal operations}

These include passive and active surveillance and reporting of cases, and treatment when necessary.

\section{Reservoir control}

No effective control measure is currently available against sylvatic reservoir hosts.

\section{Vector control}

Peridomestic spraying in the Rimac valley of Peru resulted in a pronounced reduction in the numbers of $L u$. peruensis and $L u$. verrucarum over a complete season. Insecticide spraying inside dwelings and in peridomestic areas during outbreaks and just before the transmission season might reduce transmission rates.

\section{Evaluation}

This should include passive or active detection of cases among preschool children, preferably in combination with sequential skin testing. 
Figure 6.

Geographical distribution of cutaneous and mucocutaneous leishmaniasis in the New World

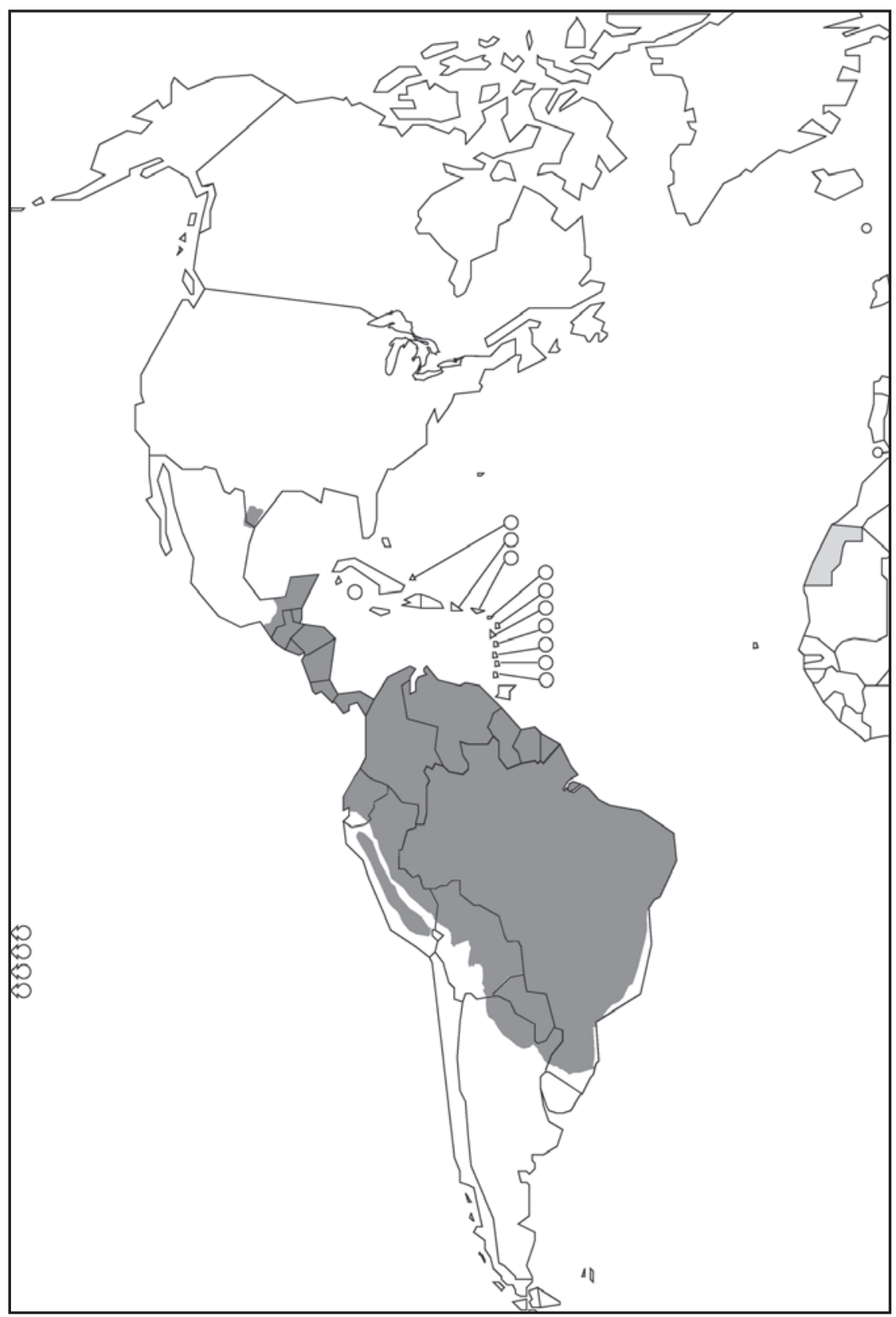




\subsection{Cutaneous leishmaniasis caused by L. guyanensis}

Geographical distribution (see Table 5 and Figure 6)

Argentina, Bolivia, Brazil (Acre, Amapá, Amazonas, Pará and Roraima states), Colombia, Ecuador, French Guiana, Guyana, Peru, Suriname and Venezuela

\section{Epidemiological features}

Transmission is associated with activities in forests; thus, peridomestic transmission occurs only when dwellings are close to the forest. There is transmission throughout the year, but the risk varies according to season. Transmission can occur during the day. Infection rates are high, reaching 90\% in exposed military units. Edentates, i.e. sloths (Choloepus didactylus) and anteaters (Tamandua tetradactyla), are the primary reservoir hosts. Marsupials, which are the secondary reservoir hosts, can be of major importance in areas where the ecology has been altered. Rodents are infected but probably do not play a significant role as reservoir hosts. The proven vectors are $L u$. umbratilis, which rests on trunks of large trees in large numbers, and $L u$. anduzei. Lu. whitmani has been found to be infected, but its role as vector is uncertain. The anthropophilic vector $L u$. longiflocosa has been implicated in the sub- Andean region of Colombia.

\section{Minimal operations}

These include passive and active detection of cases, reporting and treatment of all cases.

\section{Reservoir control}

No practical measure is currently available for control of edentates, but elimination of Didelphis marsupialis is of possible value in urban or semiurban areas.

\section{Vector control}

Short-term reduction of $\mathrm{Lu}$. umbratilis populations has been achieved by spraying insecticides on tree-trunk resting sites. This method might provide short-term protection over limited areas for people working in the forest. In French Guiana, clearing forest for $300 \mathrm{~m}$ around villages, combined with insecticide application in the cleared zones, resulted in reductions in both the sandfly population and the annual number of new leishmaniasis cases. 


\section{Evaluation}

Assessment can be made by passive and active detection of cases and regular estimation of case rates. Evaluation of risk should focus on people who have recently arrived in the transmission area.

\subsection{Cutaneous and mucocutaneous leishmaniasis caused by $L$. panamensis}

\section{Geographical distribution (see Table 5 and Figure 6)}

Colombia, Costa Rica, Ecuador (Pacific littoral), Guatemala, Honduras, Nicaragua and Panama

Epidemiological features. As in the case of cutaneous leishmaniasis caused by L. guyanensis, transmission is associated mainly with activities in deep jungle and the opening up of new land, but there is some evidence that it also occurs in areas where the original forest has been removed. The risk for infection is not uniform over all jungle areas, but infection rates of $60-70 \%$ have been observed in small population groups. In Colombia, transmission can also occur in the peridomestic environments of Andean valleys with coffee plantations. The parasite can cause mucocutaneous leishmaniasis in a small proportion of cases.

The principal reservoir host, is the two-toed sloth, Choloepus hoffmanni; the three- toed sloth, Bradypus griseus, is also an important reservoir host and is said to be the principal reservoir host in Costa Rica. Various mammalian species, incuding the domestic dog and several rodent species, have been found to be infected but are unlikely to be important reservoirs. One vector species, $L u$. trapidoi, is the proven vector of probably major epidemiological relevance, but $L u$. ylephiletor, Lu. gomezi and Lu. panamensis have also been found to be naturally infected.

\section{Minimal operations}

These include passive and active case detection, recording of cases and treatment.

\section{Reservoir control}

No effective disease control measure is currently available against sylvatic reservoir hosts.

\section{Vector control}

As for the control of cutaneous leishmaniasis caused by L. guyanensis, clearing the forest for $300 \mathrm{~m}$ around villages, with application of insecticide in the cleared zones, could reduce sandfly populations and consequently reduce the 
number of new cases. Repellents can be useful under certain circumstances, e.g. when entering the forest for occupational or recreational purposes. In areas with peridomestic transmission, the use of insecticide-treated nets and spraying might help control transmission.

\section{Evaluation}

Passive and active detection of cases and regular estimation and monitoring of incidence rates should be performed.

\subsection{Cutaneous and mucocutaneous leishmaniasis caused by $L$. braziliensis}

\section{Geographical distribution (see Table 5 and Figure 6)}

Argentina, Belize, Bolivia, Brazil, Colombia, Costa Rica, Ecuador, French Guiana, Guatemala, Honduras, Mexico, Nicaragua, Panama, Paraguay, Peru and Venezuela

\section{Epidemiological features}

lnfection is associated with jungle activities and particularly the clearing of land; transmission occurs throughout the year but with seasonal fluctuations. The risk for infection is not uniform and may be high in population groups that penetrate the forest. Both sylvatic and peridomestic foci occur; other foci may be found close to remnants of forests, surrounding secondary forest and even close to coffee and cocoa plantations. A proportion of patients $(<5 \%)$, varying from area to area, develop mucocutaneous leishmaniasis after periods

of a few weeks to over 20 years. The reservoir hosts incriminated are various species of edentates, opossums, rodents and also dogs. $\mathrm{Lu}$. wellcomei, $\mathrm{Lu}$. whitmani, Lu. ovallesi, Lu. carrerai carrerai and many others have been proven to be vectors (see Table 5).

\section{Minimal operations}

Passive and active detection and reporting of cases and treatment should be conducted. In peridomestic foci with high transmission rates, estimates of prevalence of infection among dogs might be considered.

\section{Reservoir control}

No effective control measure is currently available against sylvatic reservoir hosts.

\section{Vector control}

If the population at risk is concentrated in relatively small areas, residual house spraying may be beneficial and economically justified. Repellents can 
be useful under certain circumstances, e.g. for people entering the forest for occupational or recreational purposes. In areas with peridomestic transmission, the use of insecticide-treated nets and spraying might help control transmission.

\section{Evaluation}

For evaluation of anti-sandfly measures, see section 3.5.3.

\subsection{Cutaneous leishmaniasis caused by L. mexicana and related species}

\section{Geographical distribution (see Table 5 and Figure 6)}

Argentina, Belize, Bolivia, Brazil, Colombia, Costa Rica, Ecuador, French Guiana, Guatemala, Mexico, Peru, Suriname, USA and Venezuela

\section{Epidemiological features}

The foci have strikingly varied landscapes: wet secondary forest, or igapó, where L. amazonensis is found in Brazil, drier forest on the Yucatan peninsula and dry scrub forest on the Texas (USA)-Mexico border. Generally the disease is of low endemicity, with scattered cases. A small percentage of patients develop diffuse cutaneous leishmaniasis. Proven vectors are Lu. olmeca olmeca, Lu. ayacuchensis, Lu. flaviscutellata and Lu. longipalpis; various other species are suspected. The reservoir hosts are a variety of forest rodents and marsupials.

\section{Minimal operations}

These include passive and active detection and reporting of cases, and treatment when indicated.

Reservoir control

No effective control measure is currently available against sylvatic reservoir hosts.

\section{Vector control}

Because the disease is dispersed and generally not severe, anti-sandfly measures are only rarely economically justified. 


\subsection{Cutaneous leishmaniasis caused by $L$. infantum}

Geographical distribution (see Tables 4 and 5)

Cases have been detected in the New World (Colombia, Costa Rica, El Salvador, Honduras and Nicaragua) and in the Old World (Albania, Algeria, Armenia, Bosnia and Herzegovina, Bulgaria, China, Croatia, Cyprus, France, Greece, Israel, Italy, Kyrgyzstan, Lebanon, Macedonia, Malta, Mauritania, Monaco, Morocco, Portugal, Slovenia, Spain, Tunisia and Turkey).

\section{Epidemiological features}

Cutaneous lesions usually present as small nodules or ulcerations. Familial cases have been reported. In the New World, the proven vector is $\mathrm{Lu}$. longipalpis, and the domestic dog is the reservoir host.

\subsection{Cutaneous leishmaniasis caused by other New World species}

(see Table 5)

Leishmania $(V$.) lainsoni causes cutaneous leishmaniasis, usually presenting as a single ulcer. The disease is found in Brazil (Acre, Amapa, Para and Rondonia states), Bolivia (subtropical areas), French Guiana and Peru (tropical areas). The vectors are Lu. ubiquitalis in Brazil and Peru and $L u$. nuneztovari anglesi in Bolivia. The reservoir in Brazil is Agouti paca.

Leishmania (V.) lindenbergi causes cutaneous leishmaniasis in Brazil (Para State). Neither the vector not the reservoir is known.

Leishmania $(V$.) naiffi causes a single, small, self-limiting lesion, with no mucocutaneous leishmaniasis. It is found in Brazil (Rondonia State), Suriname and French Guiana. The vectors are Lu. squamiventris, Lu. paraensis and $L u$. ayrozai, and the reservoir is the armadillo (Dasypus novemcinctus).

Leishmania (V.) shawi causes skin ulcers. It is found in Brazil, in the Atlantic rainforest, the northeast and Para State to the south of the Amazon River. In primary forest, the vector is $\mathrm{Lu}$. whitmani. The reservoirs are monkeys (Cebus appeal, Chiropotes satanus), Procyonids (Nasua nasua) and sloths (Bradypus tridactylus and Choloepus didactylus). 



\section{Organization of control}

The main elements to consider when organizing a national leishmaniasis control programme according to the distribution pattern of the disease are national control plans, coordination mechanisms (intersectoral committee, a national task force), effective social mobilization and communication strategies, resource mobilization, monitoring and evaluation strategies and operational research. A national control programme includes all levels of the health services, and Table 6 shows a possible distribution of tasks at different levels

of the national health system, although the framework should be adapted by each country.

Table 6.

Possible distribution of tasks for the control of leishmaniasis within a national health system

\begin{tabular}{ll}
\hline Level & Activity \\
\hline Community (local) & Identifying and referring clinical suspected cases \\
& Following up treated patients \\
& Contributing to the identification of sites of high \\
transmission (at primary health-care level) & Assisting in spraying \\
& Installing traps, impregnated curtains and other measures \\
& Assisting in control of animal reservoir hosts \\
& Facilitating community participation in vector control \\
& Identifying and referring clinically suspected cases \\
Contributing to the identification of sites of high \\
transmission \\
Diagnosis (serological) \\
Treatment \\
Following up treated patients
\end{tabular}




\begin{tabular}{l} 
Hospital (district and regional) \\
Diagnosis (serological and parasitological) \\
Treatment \\
Developing and implementing plan \\
Surveillance, monitoring and supervision \\
Ensuring supplies \\
Health education and communication \\
Coordinating social mobilization \\
Organizing operational control plans \\
Implementing vector control \\
Investigating outbreaks \\
Coordinating with districts \\
Defining the national strategy and control plan \\
Financing \\
Procuring and distributing supplies \\
Disseminating technical information \\
Information, education and communication and behaviour \\
change communication (e.g. radio, television, posters) \\
Technical support \\
Surveillance, monitoring and evaluation \\
Creation of national task force, technical advisory group \\
Quality control of diagnosis and treatment \\
Monitoring drug resistance and pharmacovigilance \\
Multisectoral coordination \\
Preparing reports \\
\hline
\end{tabular}

\subsection{Control of leishmaniasis as part of primary health care}

Effective primary health care requires maximum involvement of the community. The form of this involvement depends on sociological and cultural characteristics, such as religion, traditions and other local factors. Elements such as economic status, level of education and administrative infrastructure also play a role. As a consequence, the implementation of primary health care differs greatly from country to country and, within countries, from one locality to another.

Primary health-care services for leishmaniasis in endemic countries are often severely short of skilled personnel, facilities and finances, and certain technical compromises may have to be made in conducting leishmaniasis control 
activities at primary health-care level. The amount of time required for implementation should not be underestimated.

\subsubsection{Community participation}

Community participation should not be interpreted as mobilizing the local population as cheap manpower for land-clearing or for killing animal reservoir hosts. In order to achieve the long-term commitment of a community, its members must have a clear understanding of the advantages they will gain from the proposed control measures and activities. As each individual has his or her own priorities in health matters, a programme that covers several diseases is usually more interesting to a community than projects for the control of a single disease. The 'package' should be defined in such a way that most members of the community can clearly recognize their personal interests in the objectives of the programme.

The tasks entrusted to the community should be within its capacity. Community health workers can be useful in referring clinically suspected patients to an appropriate centre for diagnosis and treatment and, after their return to the community, improve drug compliance, monitor side-effects and ensure that patients attend for follow-up examinations. Cooperation between community members and specialized teams (e.g. for insecticide spraying) can be facilitated by community health workers.

\subsubsection{Social mobilization and communication}

Social mobilization for behavioural change at community level requires effective communication strategies. Regular dialogue between the population and professional health personnel is essential. A good way of obtaining genuine commitment from a community is to form community health committees. Professional health personnel must be prepared to support and guide communities rather than give orders.

\subsection{Definition of national plans}

The overwhelming majority of Leishmania-endemic countries ( 85 of the 98) are developing countries, and notification of leishmaniasis cases is mandatory in only one third of these countries. Much of the affected population in endemic areas has no access to state health programmes; diagnosis and treatment may be unavailable because of the time and cost of travelling to medical centres or ignorance about the availability of those services. Most of the health budget in these countries is spent on curative services, so that disease prevention programmes face a permanent shortage of qualified personnel, transport and funds. While improvements have been made in health service infrastructure and technologies, implementation of programmes for active 
case searches, primary health education and training of health workers in diagnosis and case management are needed. The effectiveness of active case detection can be improved by training health workers and by introducing rapid diagnostic tests like rK39 and new, shorter therapeutic schemes based on medicines such as liposomal amphotericin B.

The lack of technology and trained personnel required to incriminate vectors and animal reservoirs remains a serious obstacle to determining appropriate intersectoral approaches to control in many countries. At present, vector control is applied in only a few countries, and animal reservoir control, with proper planning, monitoring and evaluation, is often absent.

\subsubsection{Purpose and implementation of national control programmes}

Any national control programme, no matter how small, should be based on a carefully defined plan with a concrete description of objectives, methods and schedules (Table 7) and a budget (Table 8). Researchers and disease control programme managers should participate in their design, and the plan should be formally adopted by the ministry of health and become part of the national health policy. Government acceptance of a control programme and of its budget implies a political and administrative commitment that is likely to guarantee its sustainability.

Table 7.

Steps in the development of a national programme for the control of leishmaniasis

1. Situation assessment

- Carry out desk assessment of published and unpublished reports to review disease types, tentative geographical distribution, estimated population exposed, potential vectors and animal reservoir hosts and their distribution.

- Analyse cost-effectiveness and feasibility of various control approaches.

- Assess the data management and reporting system.

- Conduct spot surveillance (local prevalence, suspected vectors and animal reservoir hosts)

\section{Preparation of national programme}

- Identify control requirements; define valid approaches to control on the basis of available data.

- Establish an intersectoral coordination and technical advisory body.

- Make an inventory of personnel and facilities; estimate training needs and equipment requirements, identify treatment centres and estimate drug requirements.

- Introduce passive surveillance in health centres in endemic areas to obtain a rough indication of prevalence and to improve the chances of individual cure. Make notification of leishmaniasis mandatory. 
- Establish the feasibility of the programme in relation to other health needs.

- Elaborate a national control strategy and plan.

- Solicit funds from national and international sources, and mobilize resources.

3. Implementation

- Continue mobilizing resources to support control activities.

- Establish a management system for drugs and other medical supplies.

- Conduct training; distribute circulars, reference publications and manuals; organize a national reference laboratory.

- Conduct health education and social mobilization including behavioural change communication; inform the population, and prepare visual aids.

- Strengthen laboratory services and establish links with the national reference laboratory.

- Strengthen the disease surveillance system; collect, analyse and disseminate feedback.

- Establish relations with international parasitological, entomological and zoological reference laboratories.

- If appropriate, apply control measures against vectors or animal reservoirs.

\section{Monitoring and evaluation}

- Assess progress of programme: prevalence and incidence, geographical boundaries, animal reservoir density and sandfly density.

- Adjust the programme and the plan on the basis of results.

Table 8.

\section{Budget framework for a national programme for the control of leishmaniasis}

\section{Item}

Cost

1. Programme development and evaluation

National coordination (desk study, intersectoral

coordination, training, reporting, international liaison

with reference centres and donor agencies)

Consultants

Training (national seminars, information circulars, books, periodicals, manuals)

Supplies and equipment (office materials)

Transport (vehicle(s), maintenance, petrol)

Travel (national and international)

$\times$ person-months: 
2. Medical surveillance

Personnel (medical officers, biologists, nurses, auxiliaries, laboratory personnel, field staff)

Consultants

Health education (posters, hand-outs, videos, local community meetings)

Supplies and equipment (diagnostic equipment and reagents, microscopes, refrigerators)

National reference laboratory (serological equipment, sampling, sample storage)

Drugs

Transport (four-wheel or two-wheel vehicles, maintenance, petrol)

3. Control of vectors and animal reservoirs

Personnel

Consultants

Supplies and equipment (traps, spraying equipment, insecticides, poisons, laboratory equipment, camping equipment, repellents)

Health education

Transport (vehicles, maintenance, petrol)

$\times$ person-months:

4. Miscellaneous

$\times$ person-months:

All expenses are charged as programme costs; however, in reality, this does not always imply a budget increase, as certain items or personnel may already be available or budgeted in other programmes by the department concerned

A plan is also indispensable as a technical baseline for measuring progress and regularly evaluating the programme. The health personnel involved should have access to the plan and a clear understanding of their roles in the overall programme. Finally, the plan is an important document for mobilizing national resources and, where applicable, for approaching potential donor agencies. 


\subsubsection{Collection of epidemiological data}

Information on the epidemiology of leishmaniasis in endemic countries has improved, and this information is now widely available from $\mathrm{WHO}$, which supported collection of epidemiological data, registration of cases in each country and organization of regional meetings of experts. Valuable tools such as the e-compendium (http://apps.who.int/tools/geoserver/www/ecomp/ index.html) and national profiles for gathering information on the types of disease, their prevalence, epidemiological features, distribution and access to medicines in each country, are also available. These, with work done by research groups in universities and health ministries of Leishmania-endemic countries, provide a clearer overview of leishmaniasis at country level.

\subsubsection{Definition of control strategies and activities}

The collected information should be translated into action to control the disease. Specific strategies and activities are outlined in sections 3-8. The control programme should be evaluated periodically, with tailored adjustment of strategies and activities.

\subsubsection{Intersectoral coordination}

Leishmaniasis cannot be controlled successfully without the involvement of different levels of administration, including the ministry of agriculture. The intersectoral committee should advise on and coordinate control activities. Personnel in a wide variety of disciplines in the health, social and biological sciences should be involved, including physicians and researchers in entomology, mammology, parasitology, anthropology and veterinary sciences.

\subsubsection{Formal adoption of the national control strategy or plan}

Countries that have not already established a leishmaniasis control programme should do so as soon as possible. The programme must be supported by the WHO regional office in order to coordinate international control strategies. Although the epidemiology of leishmaniasis may be unique in each region, control policies should be standardized as much as possible.

Establishment of local networks, such as this existing in the East Mediterranean Region, is a good strategy for coordinating joint actions and sharing technically skilled health personnel. The involvement of experts from different countries in the same region should be encouraged in order to maximize collaboration in the design, implementation and evaluation of control programmes. 


\subsection{Surveillance}

Surveillance involves systematic collection, analysis and interpretation of outcome-specific data for use in the planning, implementation and evaluation of public health practice. It includes continuous data collection, timely analysis and dissemination of data and a functional capacity to undertake effective prevention and control activities on the basis of this information.

The components of a surveillance system include: (i) entities such as clinics or hospitals for the collection of primary data (e.g. numbers of visceral leishmaniasis and PKDL cases), based on established case definitions and structured reporting forms (paper or electronic); (ii) officially mandated routes and frequencies of reporting, collation and monitoring; (iii) defined triggers for public health action (e.g. the number of visceral leishmaniasis cases within a geographically or otherwise defined catchment area that triggers an outbreak investigation or indoor residual spraying); (iv) continuous monitoring and evaluation of the system for timeliness and effectiveness; and (v) regular analysis of collated data for trends over time and space.

Skills in data management should be strengthened at programme level. When disease surveillance is mandated by the government (e.g. notification of cases of the disease is required by law), the system is likely to be far more complete than when reporting is voluntary. Therefore, in endemic countries, timely notification of leishmaniasis cases to public health authorities should be obligatory.

Although a surveillance system is unlikely to capture $100 \%$ of cases, it is essential in order to assess differences in reporting over time and between surveillance sites for interpretation of data. In the design of such a system, evaluation is greatly facilitated by the use of indicators of effectiveness, which are collected and monitored as part of surveillance. Surveillance data should also be collected from the private sector and nongovernmental organizations. A surveillance protocol or guidelines should be designed for monitoring the burden of leishmaniasis and trends in Leishmania-HIV coinfection and for evaluating the effectiveness of control measures.

\subsection{Pharmacovigilance}

Pharmocovigilance systems are weak in most endemic countries and should be strengthened. A multi-disease approach would appear to be the most efficient approach. Although considerable work has been done to find new medicines for leishmaniasis, the effectiveness of treatment programmes has been affected by problems of toxicity, adherence and treatment response, particularly in low-income countries. These are relatively common phenomena but are rarely reported systematically in most countries. Adverse reac- 
tions related to use of antileishmanial medicines can reduce confidence in the safety of these medicines, altering patient adherence to therapy. They may also reduce the effectiveness of treatment, by increasing morbidity and mortality, and the effectiveness of treatment programmes, by increasing the risk for secondary drug resistance. The strengthening of pharmacovigilance for antileishmanial medicines is therefore urgent.

Pharmacovigilance systems are usually focused on side-effects, adverse reactions and drug interactions. Their scope should, however, be broadened to address the growing problem of substandard and counterfeit medicines and to monitor the development of Leishmania resistance to new medicines that may be barely beyond the clinical trial stage. Routine monitoring of cure rates is recommended. Lessons should be learnt from experiences in the use of different medicines. It is important to distinguish between adverse events and adverse drug reactions in order to determine the severity and the relation of adverse events to a product. Once an adverse event has been detected, its maximum severity should be established and graded according to international standards. It should be established whether the event was caused by the product or by other medicines administered concomitantly (or the involvement of other illnesses). Serious adverse events should be investigated immediately and reported in a timely manner.

Pharmacovigilance systems should include passive follow-up of postmarketed products with spontaneous reporting protocols and use of standard pharmacoepidemiological methods. Post-authorization follow-up can be passive, with continuous reporting of adverse events and re-evaluation of the risks and benefits of particular medicines. The advantages of a passive reporting system include low cost, simplicity and the possibility for continuous detection of rare events and monitoring of safety. Passive reporting systems could be developed or strengthened by using designated sentinel facilities, such as health centres and targeted populations. A routine pharmacovigilance system for leishmaniasis treatment could initially include data on severe events and those deemed to be related to use of antileishmanial drugs. Establishing a system for passive post-authorization follow-up as a basis for signal detection should be a long-term commitment of national governments and health workers. National drug regulatory agencies should work closely with national control programmes against leishmaniasis and other diseases such as malaria to provide stewardship. Multidisciplinary national pharmacovigilance centres should be established and their capacity strengthened to analyse data on safety regularly in order to identify and review signals, generate hypotheses and ensure regular reporting and feedback. The pharmacoepidemiological methods used should include post-licensing clinical trials to assess the effectiveness and safety of medicines, case-control studies and 
active population-based evaluation of marketed products already authorized for use.

Substandard medicines have led in the past to avoidable deaths and iatrogenic side-effects. Quality assurance and regulatory mechanisms are critically important.

\subsection{Monitoring and evaluation}

Countries should evaluate their programmes regularly for compliance with the original objectives and procedures. Parameters should be established for elements of the programme, such as vector management, case detection and treatment, including input, processing, output, outcome and impact indicators as well as definition of the targets to be achieved within a predetermined time. Information on programme coverage, rates of morbidity and mortality, therapeutic responses to medicines and detection of new transmission foci is particularly important. Data for these indicators are usually collected through a national surveillance system (or at sentinel sites) and health management information systems.

Monitoring involves routine tracking of programme performance by recordkeeping, regular reporting, surveillance and periodic surveys. The objectives of monitoring are to verify the progress or status of implementation, ensure accountability, detect problems and constraints, promote evidence-based planning and provide timely feedback so that adjustments can be made as needed. Monitoring indicators include input, process and output indicators.

Evaluation involves periodic assessment of changes in targeted outcomes or results that can be attributed to a programme. The objectives of evaluation are to relate a particular outcome or (health) impact directly to a particular intervention after a certain time, determine the value or worth of a particular project or programme, link any two parts of the monitoring and evaluation framework (inputs, processes, outputs, outcomes or impact), measure the effectiveness of the programme and provide reliable information on progress in controlling leishmaniasis that can be used at local, national or international level.

The main objectives of monitoring and evaluating a leishmaniasis control programme are to:

- collect, process, analyse and report or disseminate information relevant to leishmaniasis;

- verify that activities have been implemented as planned to ensure accountability and address problems in a timely manner;

- provide feedback to relevant authorities to improve future planning; and 
- document whether the planned strategies have achieved the expected outcomes.

An example of a plan for monitoring and evaluating a leishmaniasis control programme is given in Table 9. ${ }^{9}$

Table 9.

Example of a framework for monitoring and evaluation

Measure Main indicators Baseline Target Source and interval or frequency

$\begin{array}{ll}\text { Input } & \begin{array}{l}\text { Finance, strategies, national } \\ \text { control plans, guidelines }\end{array} \\ \text { Process } & \begin{array}{l}\text { Human resources, training, } \\ \text { supplies and commodity (rapid } \\ \text { diagnostic tests, } \\ \text { antileishmanial medicines) }\end{array}\end{array}$

Output Service delivery, knowledge, skills; number or proportion of health facilities in endemic districts that provide diagnosis services for VL

- number of cases detected

- number or proportion of health facilities in endemic districts providing treatment for VL or CL;

- number or proportion of PKDL cases treated

- number or proportion of VL cases screened for HIV

- number or proportion of HIVpositive VL cases treated

- number of insecticidetreated nets distributed in endemic districts

- number or proportion of houses with indoor residual spraying

Outcome Proportion of early diagnosis among all detected cases

Proportion of treated VL cases cured (cure rate)

Case fatality rate
Annual programme reports

Health management information system

(quarterly)

Health management information system, surveillance reports (quarterly)
Surveillance or health management information system reports (annually) 
Proportion of HIV-positive VL cases cured

Proportion of households with at least one insecticide-treated net

Proportion of children $<5$ years old who slept under an insecticide-treated net the previous night

Impact Morbidity attributed to leishmaniasis (incidence rate, prevalence)

Mortality attributable to leishmaniasis
Surveillance or health management information system reports (annually)

Community-based surveys (3-5 years)

Demographic and health survey, community-based surveys ( 5 years)

Health facility survey, health management information system

Health facility survey, health management information system, community-based surveys

VL, visceral leishmaniasis; CL, cutaneous leishmaniasis; PKDL, post-kala-azar dermal leishmaniasis

9 More details are provided in: WHO SEARO. Guidelines and standard operating procedures for kala-azar elimination in South-East Asia countries. Trial edition, 2007. Available at: http:// www.searo.who.int/LinkFiles/Kala_azar_VBC-85_Rev_1.pdf. 


\section{International coordination}

\subsection{Reporting}

For effective worldwide control of leishmaniasis, international coordination between stakeholders is crucial. Liaisons between countries and groups of countries (at regional and interregional levels) is needed for exchange of technical data and biological material (see section 2.3), to ensure that control methods and principles are up to date. International coordination should serve to motivate and support everyone involved in leishmaniasis surveillance and control. It should also encourage new partners to join, for an eventual global alliance for the control of leishmaniasis. International coordination is needed to disseminate information about activities, present a common vision for control and outline clear expectations of progress. International coordination should address collaboration at the following levels:

- national programmes: sharing common strategies and team spirit;

- nongovernmental organizations and other institutions: collaborating closely with national programmes and with each other;

- research institutes: sharing efforts and collaborating with national programmes and nongovernmental organizations at field level;

- donors: supporting all required activities in a timely fashion at multilateral or bilateral level;

- international agencies: coordinating activities at international, regional and national levels; and

- regional and global networks.

International coordination should occur at both the technical level and on all issues related to advocacy and awareness-raising.

\subsection{Technical partners}

Technical partners include nongovernmental organizations, research institutes and national programmes involved in the control of leishmaniasis. Collaboration among these partners should respond to research, surveillance and control needs. The organization and implementation of control activities 
in countries should be strengthened; research institutes should be encouraged to fund and implement research on medicines, diagnosis and prevention; information management and dissemination should be harmonized and exchange of technical information facilitated. Furthermore, training should be provided for people involved in leishmaniasis activities. Eventually, international technical collaboration should lead to definition of a common approach to control. Greater international and national technical collaboration is necessary for obtaining knowledge about the burden of the disease, including economic aspects; implementation of research at field level; vector control issues; the availability and accessibility of medicines; pharmacovigilance and monitoring drug resistance; and the coordination and implementation of drug development and clinical trials.

Factors that restrict the control of leishmaniasis should be addressed as a priority. Recognizing that the operational capacities of national programmes vary greatly according to the financial resources and political stability of a country, it is important to retain good emergency preparedness capacity at international level, so that aid to national programmes in difficulty can be provided rapidly and in order to establish a coordinating body at national level. As the tools for control of leishmaniasis are relatively sophisticated, sufficient numbers of trained technicians must be available. Cross-border collaboration for coordination and periodic exchange of information will support control efforts.

In order to address the distinctively regional characteristics of leishmaniasis, regional or subregional programmes should be strengthened for harmonization and centralization of epidemiological surveillance, exchange of information between national programmes, research institutes and nongovernmental organizations and standardization of control methods and techniques. Regional technical networks are already exchanging knowledge and information through $\mathrm{WHO}$, and training courses and periodic regional meetings organized and led by WHO continue to take place in all regions.

\subsection{Intercountry programmes for advocacy and awareness-raising}

It may be advantageous for neighbouring countries to organize joint control programmes. Strong advocacy and awareness-raising programmes to increase the visibility of leishmaniasis control efforts must be seen as a priority. Advocacy is particularly important to ensure adequate continuing commitment from donors and national governments, without which worldwide control cannot succeed. Regional or subregional control programmes are more likely to attract funds than a single country. WHO regional and country offices should play a proactive role in promoting interinstitutional 
collaboration to control leishmaniasis and to build control capacity at both country and regional level.

\subsection{International standards}

International standards for control criteria and technical methods are useful for comparing different approaches. This applies particularly to the international code for labelling parasite isolates (see section 2.3 and Annex 1), to the use of reference strains of Leishmania (available from the WHO Collaborating Centre at the Université de Montepellier, France) and to a limited extent to skin test antigens. Quality assurance of tests can be promoted by exchange of reference sera and standard antigens. 



\section{Health education and training}

\subsection{Health education}

Health education is a core element in implementation of any disease prevention and control programme. Multidisciplinary working groups should be established. The target group for health education might include public health managers, health staff, community health workers and leaders, people living in endemic areas and patients. The main objectives of health education in leishmaniasis control include:

- achieving strong political commitment for control;

- enabling the community to obtain clear, correct information (preferably in their own language) and develop their capacity to make the right decisions; educating local communities, especially women, about control measures and educating and involving medical institutions in educational plans;

- enabling the community to understand problems objectively and to analyse them;

- positively changing behaviour and practice, motivating and leading the community in the right direction to effect the desired change; and

- establishing links and collaboration with people concerned with health promotion and disease control.

Decision-makers should be sensitized about the importance of leishmaniasis as a public health problem so that they will support and defend control programmes. In endemic areas, training courses for health authorities and health personnel should be organized before they begin work. Medical doctors and laboratory technicians should take part in health education programmes, as they are usually unfamiliar with the disease. Primary health education programmes should be organized for community health workers and leaders, schoolteachers and communities in endemic areas so that thay are able to recognize the disease and guide patients on what to do, to be proactive and to participate in prevention and control campaigns. Health education applies equally to personnel in nonendemic areas where infections are seen in people who have travelled to endemic areas. Countries should develop appropriate health education and behavioural change communication strategies and plans on the basis of the local context. 


\subsection{Training}

Health authorities and decision-makers, health staff, community health workers, community leaders, populations at risk and patients should receive training on various aspects of the disease and the control programme. Table 10 shows some of the training requirements for different personnel. It should be completed according the characteristics of the disease and the control programme in each country.

Health authorities and decision-makers should receive training in recognizing leishmaniasis as a public health problem, focusing on risk factors, preventive methods and treatment in order to support the development of multidisciplinary and interinstitutional research activities, the design of national control guidelines and issues of drug access and prevention.

Health staff, including physicians, entomologists, parasitologists, veterinarians and biologists, assigned to collaborate with the leishmaniasis control programme need special training to become familiar with the general objectives of the multidisciplinary approach to determining the epidemiological and biological characteristics of the disease, risk factors, prevention and control measures, treatment and pharmacovigilance.

Laboratory technicians require training on use of the different diagnostic methods available in the country. If a comprehensive programme is envisaged, parasite isolation by culture and molecular biology tools for diagnosis should also be included in their training.

Social scientists must be trained in the basic epidemiological and clinical characteristics of the disease. They should participate in the primary health education of people at risk. Studies to determine peoples' knowledge, attitudes and perceptions of the disease should be conducted in order to understand the use of local traditional medicine, treatment and preventive and control measures.

Community health workers should be trained to suspect cases and to refer them to a health centre for diagnosis and treatment. They should also be trained to support treatment administration and reporting of clinical evolution and drug-related adverse effects. A control manual for field personnel, describing current control techniques and methods, should be prepared in a language appropriate to the potential users and distributed. 


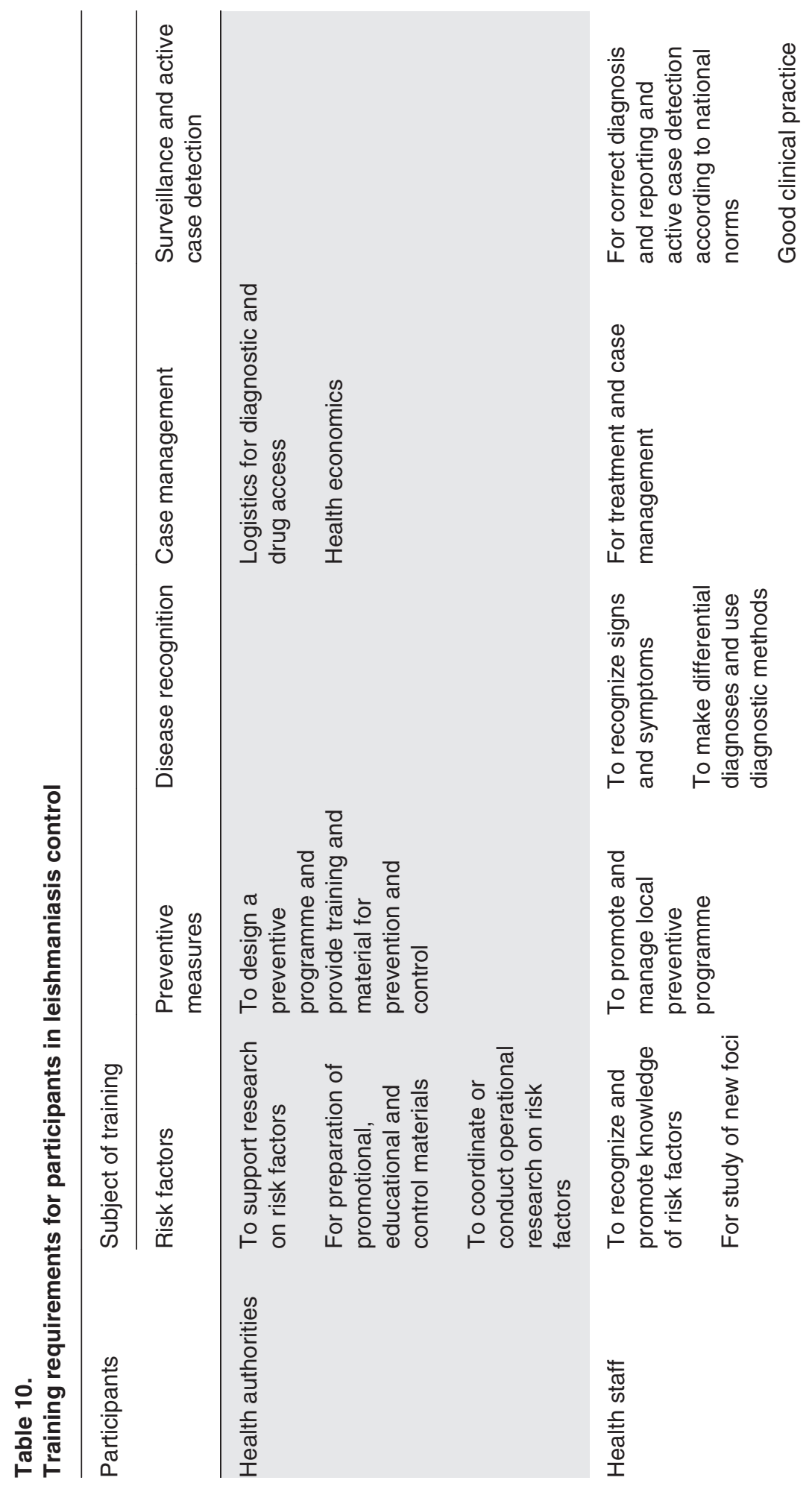




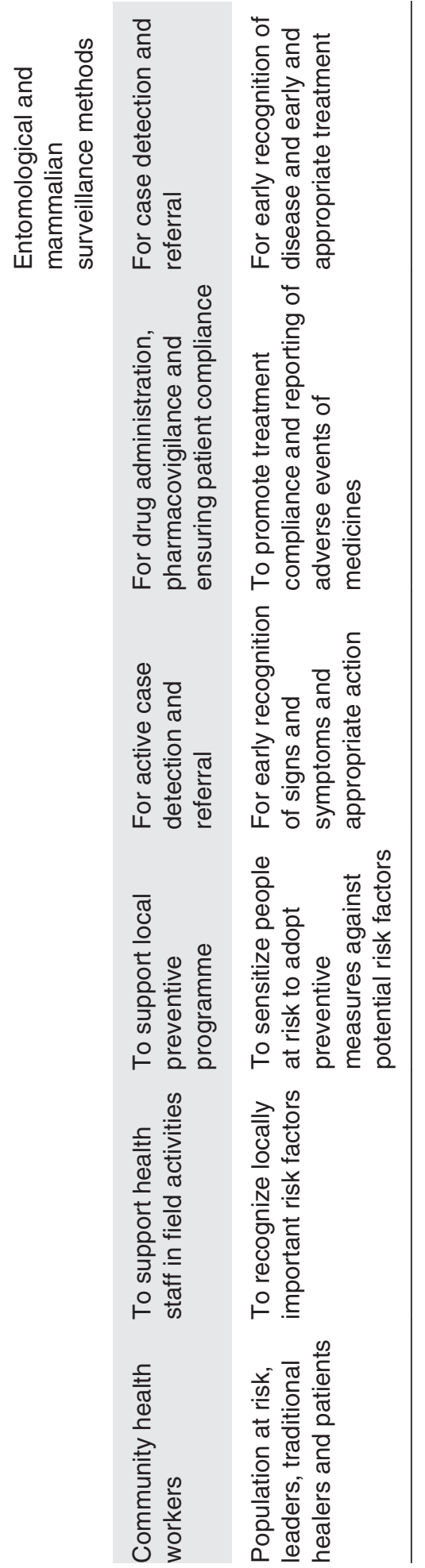


The population at risk and community leaders should be educated and trained to identify early signs and symptoms. They should have clear information on the mechanism of referral of suspected cases of leishmaniasis. It is important to emphasize the need for confirming the diagnosis and providing adequate treatment. Discussions about potential risk factors associated with the disease and preventive measures should also be part of health education and training. Local training sessions are particularly recommended, as local circumstances are best known by the participants, teachers and community leaders, and large groups of people can be reached. 



\section{Research}

\subsection{Field research}

Several aspects of the epidemiology and transmission of leishmaniasis remain unknown and require research. A concerted research effort should be made to define the population at risk and the global burden of the disease accurately. It is therefore important to identify and develop effective surveillance tools and methods for use in endemic countries.

Research on risk factors for infection and transmission are necessary, including social economic and nutritional aspects.

Steps should be taken to incriminate vectors in foci where they are unknown and in new foci as they arise. Studies on the basic biology of proven vectors are needed to judge whether control by attacking the vector is feasible, including areas where elimination or control programmes are under way. The minimum biological information should include population dynamics, with times of transmission, the range of hosts on which the vector feeds, the resting sites of adults and breeding sites.

Field studies on new foci and outbreaks of leishmaniasis should be launched without delay to determine the structure of the focus and the feasibility of control.

Long-term monitoring should be conducted of annual fluctuations in important vector populations in representative transmission sites of leishmaniasis. Examples of vectors to be included in this research are $P$. argentipes, $P$. orientalis, $P$. papatasi, $P$. sergenti, $P$. perniciosus and Lu. longipalpis.

Research and development of innovative tools for vector and reservoir control are needed, including evaluations of the cost, feasibility, acceptability and sustainability of control strategies. In particular, insecticide-based vectorcontrol tools (indoor residual spraying and insecticide-treated nets) and nonchemical ecological interventions should be evaluated, especially for anthroponotic transmission conditions.

Clinical research to evaluate new drugs and combinations of drugs to reduce the duration of treatment for all clinical forms of leishmaniasis remains a high priority. Operational research to improve the collection of information about 
treatment compliance and implementation of a pharmacovigilance system is important to better define algorithms for new treatment regimens. Extended follow-up studies are required to determine the incidence of PKDL. Studies should be conducted to determine the feasibility of using the new treatment modalities for visceral leishmaniasis and for patients coinfected with HIV.

Social-behavioural research to identify social determinants of the use of health-care services and health care-seeking behaviour is important. Identification of behavioural blocks in completing treatment and of ways to overcome these problems is important. Studies to determine the economic and social consequences of leishmaniasis are necessary for advocacy and for designing better control measures.

\subsection{Laboratory research}

Studies are needed to determine the role of asymptomatic infections and PKDL as reservoirs for transmission dynamics in visceral leishmaniasis.

Development of a leishmaniasis vaccine must be a priority, as immunization would be the method of choice for future control.

Existing knowledge and molecular tools for the identification and characterization of Leishmania parasites and sandflies should be improved and harmonized. Standardization and adoption of more sensitive, definitive molecular techniques for the diagnosis and characterization of Leishmania parasites is critical, as, in many instances, the treatment choice depends on the infecting Leishmania species. In addition, methods for determining drug resistance are needed, which are standardized and usable in endemic countries.

Research and development of rapid tests for the diagnosis of visceral leishmaniasis and tests for cure based on antigen and nucleic acid detection should be undertaken. Diagnostic tools for PKDL that can be used in the field should be developed and evaluated.

Research on more effective medicines that meet the current standards of safety remains a high priority.

International support and collaboration in terms of finance and technical expertise should be mobilized.

\subsection{Drug and vaccine research and development}

\subsubsection{What products are needed?}

The development of new medicines and vaccines for the different forms of leishmaniasis is challenging because of the variety of parasite species, 
pathology and immune responses (in particular immunosuppression). The requirements of patients and control programmes define the target product profile and the specifications of the final product. Consideration should be given to several target product profiles for medicines for different situations, including: in case management of visceral leishmaniasis, where advances in combination therapies are reducing potential treatment times;

- in control programmes for visceral leishmaniasis, with strict procedures on safety for potential use in asymptomatic people;

- for PKDL, for which the treatment options are limited and for which immunotherapy and combinations might be used;

- for Leishmania-HIV coinfection, for which the treatment options are limited;

- for localized cutaneous leishmaniasis, for which the efficacy of current medicines is limited but for which topical medicines and immunotherapy are options; and

- for treatment of complex manifestations of cutaneous leishmaniasis and for vaccines for the prevention of visceral leishmaniasis and of Old and New World cutaneous leishmaniasis.

\subsubsection{Challenges to the development and use of antileishmanial interventions}

Research is needed to translate knowledge derived from the parasite genome and biochemistry into validated parasite targets amenable to high-throughput screening to design and screen new chemical products. Improved in-vivo models are needed to help in the selection of compounds, to confirm their activity against a panel of species and to optimize pharmaceutical formulations. Translational research to transform lead molecules into candidate drugs must take into account the 'drug likeness' or 'druggability' of these molecules. This is costly, and the attrition rate is high.

As the protective immune response is an important segment of recovery from leishmaniases, immune stimulation during chemotherapy offers potential. Research to identify new immunomodulators and therapeutic vaccine candidates, especially for use in the treatment of chronic leishmaniases, requires knowledge of the mechanisms of action and development of predictive models.

Prevention of visceral and cutaneous leishmaniasis requires research on vaccines based on recombinant proteins and genetic vaccines. These contain defined antigens, which are usually those that have shown protection in experimental animals. Genetic vaccines have become an attainable goal in view of the ease of production, simple standardization, the longevity of the immune response produced and the flexibility of vaccines combining multiple genes. Registered DNA vaccines for animals would undoubtedly open the way for 
human DNA vaccines. The capacity of DNA vaccines to use both major histocompatibility class I and II pathways, thereby stimulating the induction of both CD8+ and CD4+ T cells, is important. Research on immunity will also guide the design or selection of suitable adjuvants.

For clinical evaluation, reliable biomarkers of treatment outcome are needed to reduce evaluation time and costs. Disease- and species-specific issues in designing trials for the different forms of leishmaniasis require standardization. Systematic reviews are effective in summarizing evidence and highlighting methodological issues. Trials must conform to contemporary standards of quality (good clinical and good clinical laboratory practice). Only products that have undergone scrutiny by regulatory authorities should be used. Post-regulatory studies and operational research are needed to establish the profile of a drug when used in practice and to optimize drug use under real-life conditions

\subsubsection{Input of other approaches}

Input is needed from the related fields of:

- diagnostics, for asymptomatic carriers, acute disease, test of cure and species- specific diagnosis;

- resistance, for understanding drug mechanisms, so that markers of resistance can be identified;

- pharmacokinetics, for clinical pharmacology and predictive models, in particular for drug combinations;

- clinical microbiology, for systems for monitoring and surveillance of drug susceptibility;

- human genetics, for pharmacogenomics, immune response and disease susceptibilty;

- economics, for comparing the cost-effectiveness of treatments and other interventions;

- social science, for operational research on treatment acceptability and access; and

- demography, for demographic and anthropometric profiles of populations, in order to adapt treatment regimens and supportive interventions. 


\section{Recommendations}

The Expert Committee recognizes that a major milestone was achieved with Resolution 60.13 on the Control of Leishmaniasis adopted by the World Health Assembly in 2007. The way forward outlined in the resolution was fully endorsed by the experts. The currently high morbidity and mortality from leishmaniasis is unacceptable, as affordable, effective tools for control are already available. The Expert Committee recommended as a first priority the establishment of control programmes for leishmaniasis in the affected areas, and it strongly encouraged WHO to take the lead in empowering and supplementing government programmes that are unable to address control adequately. Financial and technical support should be mobilized where necessary.

The Expert Committee also recognized the initiative and vital role of donors in the control of leishmaniasis, drawing attention to the continuing need for maintaining and expanding programmes. WHO should take a leading role in providing technical assistance to existing and new initiatives to reduce morbidity and mortality due to leishmaniasis.

Efforts should made to improve the living conditions of populations in endemic areas to reduce transmission.

\section{Parasitology}

- Isolation and identification of Leishmania are essential for treatment and control and should be strongly supported.

\section{Vectors}

- Countries should be encouraged to adopt a policy of integrated vector management for vector control in order to optimize the use of insecticides and other resources.

- Regional training programmes should be established for subordinate staff, including laboratory technicians and field entomological assistants. 


\section{Medicines, vaccines and other tools for control}

- Efforts should be made to improve access to and reduce the cost of diagnostics, medicines, insecticides and bednets, including leishmanin antigen produced according to good manufacturing practice.

- Research should be encouraged, including multicentre trials, to generate an evidence base for better therapeutic regimens for cutaneous and mucosal leishmaniasis and PKDL.

- Countries are encouraged to adopt innovative policies based on drug combinations as they become available and meanwhile to adopt the best monotherapy option.

- Efforts should be made to promote regional evaluation of locally appropriate therapies, including topical therapies for cutaneous leishmaniasis.

- The development of prophylactic as well as therapeutic vaccines and immunomodulators should be encouraged.

\section{Control programmes}

- Current managerial and operational limitations in leishmaniasis control programmes should be addressed at regional and national levels.

- National control programmes should be supported in endemic countries that do not have one.

- Leishmaniasis should be designated as a notifiable disease in all endemic countries. Leishmaniasis surveillance and pharmacovigilance should be integrated into multidisease surveillance systems.

- Further studies of the effectiveness and cost-effectiveness of control programmes should be encouraged. Methods for evaluating interventions in control programmes should be improved and validated.

- Studies are needed to understand the role of asymptomatic leishmanial infection in transmission.

- Practical laboratory tools should be developed to identify markers of infection, tests of cure and parasite resistance to medicines.

- Nonendemic countries should increase awareness of leishmaniasis as an imported disease. 


\section{Annex 1 \\ Labelling of Leishmania isolates and identification centres}

\section{Labelling of isolates}

It is recommended that each isolate of Leishmania be given a unique code showing the host (human, reservoir host or vector), country of origin, year of isolation and reference of the person who made the isolation. Further details are given in section 2.3.1. The elements of the first two parts of the code are listed in Tables A1.1 and A1.2.

Table A1.1.

Generic codes for labelling Leishmania isolates according to the international code

\begin{tabular}{llll}
\hline A & Mammalia & A & Mammalia \\
\hline ACO & Acomys (ROD) & HOP & Hoplomys (ROD) \\
AKO & Akodon (ROD) & HTX & Heterohyrax (HRC) \\
ALA & Alactagulus (ROD) & HYP & Hypoprocta (ROD) \\
ALL & Allactaga (ROD) & HYT & Hystrix (ROD) \\
ALO & Alouatta (PMT) & KAN & Kannabateomys (ROD) \\
AOT & Aotus (PMT) & LAG & Lagothrix (PMT) \\
ARV & Arvicanthis (ROD) & LEO & Leontideus (PMT) \\
ATE & Ateles (PMT) & LEP & Lepus (LGM) \\
BAS & Bassaricyon (CAR) & LER & Lestoros (MSP) \\
BRA & Bradypus (EDE) & LES & Lestodelphys (MSP) \\
BRN & Brachiones (ROD) & LUT & Lutreolina (MSP) \\
BRT & Brachyteles (PMT) & MAR & Marmosa (MSP) \\
BSS & Bassariscus (CAR) & MAS & Mastomys (ROD) \\
BUR & Burmeisteria (EDE) & MEL & Meles (CAR) \\
CAB & Cabasscus (EDE) & MER & Meriones (ROD) \\
CAC & Cacajao (PMT) & MES & Mesocricetus (ROD) \\
CAE & Caenolestes (MSP) & MET & Metachirus (MSP) \\
CAL & Caluromys (MSP) & MIC & Microtus (ROD) \\
\hline
\end{tabular}




\begin{tabular}{|c|c|c|c|}
\hline CAN & Canis (CAR) & MON & Monodelphis (MSP) \\
\hline CAP & Caluromysiops (MSP) & MST & Mustela (CAR) \\
\hline CAV & Cavia (ROD) & MUS & Mus (ROD) \\
\hline CBL & Cebuella (PMT) & MYR & Myrmecophaga (EDE) \\
\hline CEB & Cebus (PMT) & NAS & Nasua (CAR) \\
\hline CER & Cerdocyon (CAR) & NEA & Neacomys (ROD) \\
\hline $\mathrm{CHI}$ & Chironectes (MSP) & NEC & Nyctomys (ROD) \\
\hline $\mathrm{CHL}$ & Chlamyphorus (EDE) & NES & Nesokia (ROD) \\
\hline $\mathrm{CHO}$ & Choloepus (EDE) & NYC & Nyctomys (ROD) \\
\hline $\mathrm{CHP}$ & Chaetophractus (EDE) & NYT & Nyctereutes (CAR) \\
\hline $\mathrm{CHR}$ & Chiropotes (PMT) & ORY & Oryzomys (ROD) \\
\hline CLC & Callicebus (PMT) & ОтО & Ototylomys (ROD) \\
\hline CLM & Callimico (PMT) & $\mathrm{PHI}$ & Philander (MSP) \\
\hline CLX & Callithrix (PMT) & PIT & Pithecia (PMT) \\
\hline COE & Coendou (ROD) & POT & Potos (CAR) \\
\hline $\mathrm{CRI}$ & Cricetus (ROD) & PRI & Priodontes (EDE) \\
\hline $\mathrm{CRL}$ & Cricetulus (ROD) & PRO & Proechimys (ROD) \\
\hline CRM & Cricetomys (ROD) & PRV & Procavia (HRC) \\
\hline CUN & Cuniculus (ROD) & PSA & Psammomys (ROD) \\
\hline CYC & Cyclopes (EDE) & RAT & Rattus (ROD) \\
\hline DAP & Dasyprocta (ROD) & $\mathrm{RHI}$ & Rhipidomys (ROD) \\
\hline DAS & Dasypus (EDE) & $\mathrm{RHO}$ & Rhombomys (ROD) \\
\hline DEN & Dendrohyrax (HRC) & $\mathrm{RHY}$ & Rhynocholestes (MSP) \\
\hline DID & Didelphis (MSP) & SAG & Saguinus (PMT) \\
\hline DIP & Dipodomys (ROD) & SAI & Saimiri (PMT) \\
\hline DPM & Diplomys (ROD) & SIG & Sigmodon (ROD) \\
\hline DPS & Dipus (ROD) & SPE & Spermophilosis (ROD) \\
\hline DRO & Dromiciops (MSP) & TAM & Tamandua (EDE) \\
\hline DUS & Dusicyon (CAR) & TAT & Tatera (ROD) \\
\hline $\mathrm{ECH}$ & Echimys (ROD) & TOL & Tolypeutes (EDE) \\
\hline EQU & Equus (PER) & TYL & Tylomys (ROD) \\
\hline EUP & Euphractus (EDE) & URO & Urocyan (CAR) \\
\hline FEL & Felis (CAR) & URS & Ursus (CAR) \\
\hline FEN & Fennecus (CAR) & VOR & Vurmela (CAR) \\
\hline GEN & Genetta (CAR) & VUL & Vulpes (CAR) \\
\hline
\end{tabular}




$\begin{array}{llll}\text { GLI } & \text { Glironia (MSP) } & \text { XER } & \text { Xerus (ROD) } \\ \text { HEM } & \text { Hemiechinus (INS) } & \text { ZAE } & \text { Zaedyus (EDE) } \\ \text { HET } & \text { Heteromys (ROD) } & \text { ZYG } & \text { Zygdontomys (ROD) } \\ \text { HOM } & \text { Homo (PMT) } & & \\ \text { * CAR, Carnivorea; EDE, Endentata; HRG, Hyracoidea; INS, Insectivora; LGM, Lagomorpha; MSP, } \\ \text { Marsupialia; PER, Perissodactyla; PMT, Primates; ROD, Rodentia }\end{array}$

\section{B. Genera Lutzomyia and Phlebotomus}

\section{Genus Lutzomyia}

Group Migonei (MIG)

MIG L. migonei

Subgenus Nyssomiya (NYS)

AND L. anduzei

Subgenus Psychodopygus (PSY)

ANT

L. antunesi

AMA

L. amazonensis

FLA

L. flaviscutellata

AYR

L. ayrozai

INT

L. ermedia

CAR

L. carrerai carrerai

OLB

L. olmeca bicolor

DAV

L. davisi

OLM

L. olmeca olmeca

LLM

L. Ilanos martinsi

OLN

L. olmeca nociva

PAN

L. panamensis

SHW

L. shawi

PAR

L. paraensis

TRA

L. trapidoi

SQU

L. squamiventris

UMB

L. umbratilis

WEL

L. wellcomei

WHI

L. whitmani

YLE

L. ylephiletor

YUC

L. yucumensis

YUI

L. yuilli

Group

Verrucarum (VER)

Subgenus

Pintomyia (PIN)

AYA

L. ayacuchensis

PES

L. pessoai

$\mathrm{CHR}$

L. christophei

FIS

L. fischeri

EVA

L. evansi

Subgenus Psathyromyia (PSA)

NUN

L. nuneztovari anglesi

ABO

L. abonnenci

OVA

L. ovallesi

DEN

L. dendrophyla

SPI

L. spinicrassa

SCA

L. scaffi

VER

L. verrucarum

SHA

L. shannoni

Subgenus Lutzomiya (LUT)

Group Vexator (VEX) 


\begin{tabular}{|c|c|c|c|}
\hline CRU & L. cruciata & HAR & L. hartmanni \\
\hline DIA & L. diabolica & PRN & L. peruensis \\
\hline GOM & L. gomezi & SAN & L. sanguinaria \\
\hline LON & L. longipalpis & Subgenus & Viannamyia (VIA) \\
\hline REN & L. renei & TUB & L. tuberculata \\
\hline \multicolumn{4}{|c|}{ Genus Phlebotomus } \\
\hline Subgenus & Adlerius (ADL) & Subgenus & LAR (continued) \\
\hline ARA & P. arabicus & PRF & P. perfiliewi perfiliewi \\
\hline BAL & P. balcanicus & PTR & P. perfiliewi transcaucasicus \\
\hline $\mathrm{CHI}$ & P. chinensis & TOB & P. tobbi \\
\hline $\mathrm{HAL}$ & P. halepensis & Subgenus & Paraphlebotomus (PAR) \\
\hline LND & P. longiductus & ALE & P. alexandri \\
\hline NAQ & P. naqbenius & ANJ & P. andrejevi \\
\hline SIM & P. simici & CAU & P. caucasicus \\
\hline Subgenus & Euphlebotomus (EUP) & $\mathrm{CHA}$ & P. chabaudi \\
\hline ARG & $P$. argentipes & SAE & P. saevus \\
\hline Subgenus & Larroussius (LAR) & SER & P. sergenti \\
\hline$A C U$ & P. aculatus & Subgenus & Phlebotomus (PHL) \\
\hline ARI & P. ariasi & BER & P. bergeroti \\
\hline KAN & P. kandelakii & DUB & P. duboscqi \\
\hline LNG & P. longicuspis & PAP & P. papatasi \\
\hline LNP & $P$. longipes & SAL & P. salehi \\
\hline MJM & P. major major & Subgenus & Synphlebotomus (SYN) \\
\hline MJN & P. major neglectus & ANS & P. ansarii \\
\hline MJS & P. major syriacus & CEL & P. celiae \\
\hline MJW & P. major wui & GRO & P. grovei \\
\hline ORI & P. orientalis & MAR & P. martini \\
\hline PED & P. pedifer & ROS & P. rossi \\
\hline PER & P. perniciosus & VAN & P. vansomerenae \\
\hline
\end{tabular}


Table A1.2.

ISO codes for countries or territories where autochthonous cases of leishmaniasis are known or suspected, for use in the labelling of Leishmania isolates

\section{Old World}

Afghanistan

AF Libyan Arab Jamahiriya LY

Albania

AL Macedonia MK

Algeria

DZ Malawi MW

Angola

AO Mali ML

Argentina

AR Malta MT

Armenia

AM Mauritania MR

Australia

AU Montenegro ME

Azerbaijan

AZ Morocco MA

Bangladesh

BD Namibia NA

Benin

BJ Nepal NP

Bosnia and Herzegovina

BA Niger NE

Botswana

BW Nigeria NG

Bulgaria

BG Oman OM

Burkina Faso

BF Pakistan PK

Cambodia

$\mathrm{KH}$ Palestine PS

Cameroon

CM Portugal PT

Central African Republic

CF Romania RO

Chad

TD Russian Federation RU

China

CN Rwanda

RW

Côte d'Ivoire

Cl Saudi Arabia SA

Croatia

HR Senegal

SN

Cyprus

CY Serbia

RS

Democratic People's Republic

KP Slovenia

SI

of Korea

$\begin{array}{lll}\text { Djibouti } & \text { DJ Somalia } & \text { SO } \\ \text { Egypt } & \text { EG South Africa } & \text { ZA } \\ \text { Ethiopia } & \text { ET Spain } & \text { ES } \\ \text { France } & \text { FR Sri Lanka } & \text { LK } \\ \text { Gabon } & \text { GA Sudan } & \text { SD } \\ \text { Gambia } & \text { GM Syrian Arab Republic } & \text { SY } \\ \text { Georgia } & \text { GE Tajikistan } & \text { TJ } \\ \text { Germany } & \text { DE Togo } & \text { TG }\end{array}$




\begin{tabular}{|c|c|c|c|}
\hline Ghana & $\mathrm{GH}$ & Tunisia & TN \\
\hline Greece & GR & Turkey & TR \\
\hline Guinea & GN & Turkmenistan & TM \\
\hline India & IN & Uganda & UG \\
\hline Indonesia & ID & USSR & $\mathrm{SU}^{*}$ \\
\hline Iran, Islamic Republic of & IR & United Arab Emirates & $\mathrm{AE}$ \\
\hline Iraq & IQ & United Republic of Tanzania & $\mathrm{TZ}$ \\
\hline Israel & IL & Uzbekistan & UZ \\
\hline Italy & IT & Viet Nam & VN \\
\hline Jordan & $\mathrm{JO}$ & Yemen & YE \\
\hline Kazakhstan & $\mathrm{KZ}$ & Yugoslavia & YU \\
\hline Kenya & $\mathrm{KE}$ & Zaire & ZR \\
\hline Kuwait & $\mathrm{KW}$ & Zambia & ZM \\
\hline Kyrgyzstan & KG & Zimbabwe & ZW \\
\hline Lebanon & LB & & \\
\hline \multicolumn{4}{|l|}{ New World } \\
\hline Argentina & $\mathrm{AR}$ & Guyana & GY \\
\hline Belize & $\mathrm{BZ}$ & Honduras & $\mathrm{HN}$ \\
\hline Bolivia & $\mathrm{BO}$ & Martinique & $\mathrm{MQ}$ \\
\hline Brazil & $\mathrm{BR}$ & Mexico & $\mathrm{MX}$ \\
\hline Colombia & $\mathrm{CO}$ & Nicaragua & $\mathrm{NI}$ \\
\hline Costa Rica & CR & Panama & PA \\
\hline Dominican Republic & DO & Paraguay & PY \\
\hline Ecuador & EC & Peru & $\mathrm{PE}$ \\
\hline El Salvador & SV & Suriname & SR \\
\hline French Guiana & GF & Trinidad and Tobago & TT \\
\hline Guadeloupe & GP & United States of America & US \\
\hline Guatemala & GT & Venezuela & VE \\
\hline
\end{tabular}

* Former Soviet Union

The form for depositing a Leishmania strain is reproduced in Figure A1.1. 
Figure A1.1.

Information form for depositing a Leishmania strain in a collection for scientific purposes

Centre / Laboratory / Collection.

Local code:

WHO code:

Origin

Person or laboratory that isolated the strain:

Person requesting typing:

Code attributed by the provider of the strain (three letters maximum followed by a number):

Country:

Place:

Host:

Year of contamination:

Year of isolation:

A. Human strain (for positive answers, tick the box $\square$ )

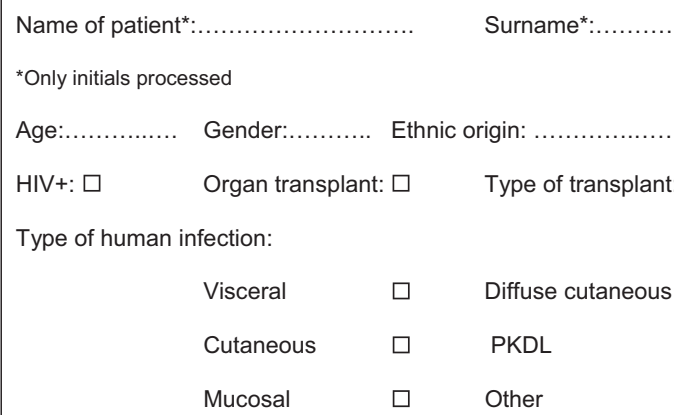

Number of cutaneous lesions: Single $\square \quad$ Multiple $\square \quad$ Number:

Original tissue samples used for isolation:

\section{B. Animal strain}

Species or common name:

Type of infection in the animal:

Visceral

Cutaneous

$\square \quad$ Mucosal

\section{Strain isolated from sandfly}


Figure A1.1.

Information form for depositing a Leishmania strain in a collection for scientific purposes (contd.)

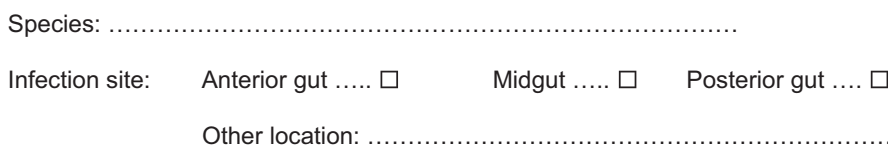

\section{Identification centres}

Centres to which Leishmania isolates can be sent for identification are listed in Table A1.3.

Table A.1.3.

Centres that have facilities for identification of Leishmania isolates and which can accept material for identification

Country Laboratory

Technique

Isoenzymes DNA

Algeria Service d'Eco-épidémiologie Parasitaire, Institut Pasteur d'Algérie, Annexe de Sidi Fredj, Dely Ibrahim, 16000 Alger

Belgium Molecular Parasitology Unit, Prince Leopold Institute of Tropical Medicine, Nationalestraat 155, 2000 Antwerp

Brazil Fundaçao Instituto Oswaldo Cruz, Avenida Brazil 4365, Manguinhos, Rio de Janeiro, CEP 21040

Colombia Centro Internacional de Investigaciones* Médicas, Colciencias, Apartado Aéreo 5390, Cali 
Programa de Estudio y Control de Enfermedades

Tropicales, Universidad de Antioquia, Calle 6252 59,

Medellin

\begin{tabular}{|c|c|c|c|}
\hline France & $\begin{array}{l}\text { Laboratoire de Parasitologie, Centre National de Référence } \\
\text { des Leishmania, } 39 \text { avenue Charles Flahault, } 34295 \\
\text { Montpellier Cedex } 5\end{array}$ & + & + \\
\hline India & $\begin{array}{l}\text { Institute of Pathology, Safdarjung Hospital Campus, New } \\
\text { Delhi } 110029\end{array}$ & $\bullet$ & + \\
\hline Israel & $\begin{array}{l}\text { Department of Protozoology, Hadassah Medical School, } \\
\text { Hebrew University, PO Box } 1172 \text {, Jerusalem }\end{array}$ & & + \\
\hline Italy & $\begin{array}{l}\text { Reparto di Malattie trasmesse da Vettori e Sanitá } \\
\text { Internazionale, Istituto Superiore de Sanità, Viale Regina } \\
\text { Elena 299, } 00161 \text { Roma }\end{array}$ & + & + \\
\hline Peru & $\begin{array}{l}\text { Instituto de Medicina Tropical Alexander Von Humboldt, } \\
\text { Universidad Peruana Cayetano Heredia, Apartado 5045, } \\
\text { Lima } 100\end{array}$ & & + \\
\hline Spain & $\begin{array}{l}\text { Sevicio de Parasitología, Centro Nacional de } \\
\text { Microbiología, Instituto de Salud Carlos III, } 28220 \\
\text { Majadahonda, Madrid }\end{array}$ & + & + \\
\hline \multirow[t]{2}{*}{ Tunisia } & Institut Pasteur de Tunis & + & + \\
\hline & $\begin{array}{l}\text { Laboratoire de Parasitologie, Faculté de Pharmacie, } \\
\text { Monastir, Tunisie }\end{array}$ & + & \\
\hline \multirow[t]{2}{*}{ USA } & $\begin{array}{l}\text { Division of Parasitic Diseases, Centers for Disease Control } \\
\text { and Prevention, Atlanta }\end{array}$ & + & + \\
\hline & $\begin{array}{l}\text { Infectious Diseases Division, Walter Reed Army Institute of } \\
\text { Research, Washington DC }\end{array}$ & $\bullet$ & + \\
\hline
\end{tabular}

* Can also perform monoclonal antibody typing 



\section{Annex 2 \\ Methods for the isolation and cryopreservation of Leishmania ${ }^{10}$}

\section{Isolation of Leishmania}

Isolation in vitro offers certain advantages over in-vivo methods. Cultures show positive results more rapidly, often in as little as 5-7 days, whereas it may take weeks or months for a lesion to appear on an animal. The materials required are less expensive, and the cultured organisms can be cryopreserved, thus reducing the time and personnel required for maintenance. The disadvantages of in-vitro isolation are that some Leishmania species are extremely difficult to culture, and sterile conditions may be difficult to attain in the field. Nevertheless, adequate conditions for in-vitro isolation can be achieved in the field with organization and a little ingenuity, for instance by using the flame of a camping gas cooker or even a cigarette lighter. Body surfaces, both human and animal, from which organisms are to be isolated should be thoroughly cleaned with alcohol or other antiseptic, and sterile instruments must be used.

Injection of isolates into the footpad of susceptible hamsters or mice and removal of samples 7-10 days later for inoculation into culture media combines the advantages of in-vivo and in-vitro methods.

Under adverse conditions, both methods of isolation should be used; if a single method must be chosen for field use, it should be the in-vivo one. Its main advantage is that strict aseptic technique is not required; its main disadvantage is that not all species of Leishmania infect any one kind of laboratory animal.

\section{Human cutaneous leishmaniasis}

Take tissue samples from the areas of the lesion most likely to contain leishmanial amastigotes. Generally, the red swollen edge of a cutaneous lesion is the best site. Several methods are used for taking samples. Biopsy of the lesion edge is a standard dermatological technique and usually requires only a local anaesthetic. The sample is excised with a small scalpel or removed with a dermatological punch. Other methods, which do not usually require local

\footnotetext{
10 Adapted from Evans D, ed. Handbook on isolation, characterization and cryopreservation of Leishmania. UNDP/World Bank/WHO Special Programme for Research and Training in Tropical Diseases, Geneva, World Health Organization.
} 
anaesthesia, include slit-skin preparations, dental broach biopsy and aspiration of the lesion edge.

Introduce the tissue sample into the culture medium. If biphasic medium is used, inoculate into the liquid portion. When the piece of tissue is large, press it against the inside of the culture vessel with a large sterile needle or similar implement. Alternatively, grind the tissue in a small, sterile tissue grinder, and inoculate the resulting liquid.

\section{Human visceral leishmaniasis}

Isolations are usually made from either bone marrow or splenic aspirates; occasionally, venous blood is used. Samples of bone marrow and spleen should be taken only by medically qualified, experienced personnel (see Annex 4). Make stained smears from the samples and inoculate culture media and animals.

The organisms from patients with visceral leishmaniasis can be difficult to cultivate. Whenever possible, try to inoculate experimental animals as well. When attempting cultivation, use a blood-agar-based medium, preferably Novy-MacNeal-Nicolle (NNN), otherwise the United States Army Medical Research Unit (USAMRU) or modilied Tobie medium. Sometimes, even when the initial isolation is successful, the organism may die when subcultured. This seems especially common when the initial isolation has been into a rich medium such as USAMRU or modified Tobie. This can often be overcome by making subcultures into less nutritionally rich media, such as NNN or a semi-solid medium such as 'sloppy Evans' or semi-solid Locke bloodagar.

\section{Isolation from mammals other than humans}

\section{Domestic dogs}

Lymph node puncture: The popliteal lymph nodes are almost invariably enlarged and parasitized in canine visceral leishmaniasis, and these are the glands most commonly sampled for diagnostic purposes. Occasionally the prescapular lymph node is punctured. The procedure is as described below, with the dog seated.

- Muzzle the dog with a non-elastic strip of cloth, $50 \mathrm{~mm}$ wide, e.g. a linen belt or bandage, tying it firmly under the jaw and a second time behind the ears.

- Lay the dog on its side; an assistant must hold its head firmly.

- Cut and shave the hair from the skin over the node. Swab the exposed skin with alcohol-soaked cottonwool pads until a pad remains clean. 
- Infiltrate the skin around the lymph node with 3-4 $\mathrm{ml}$ of $1.0 \%$ lidocaine (local anaesthetic).

- Palpate the lymph node and immobilize it between the thumb and forefinger.

- Puncture the node with a 50-mm, 19-gauge needle attached to a syringe containing $1 \mathrm{ml}$ of saline with antibiotics.

- Pull back the plunger of the syringe and, without leaving the node, draw the needle back. Then push it in another direction so as to explore all parts of the node. Withdraw the syringe and needle.

- Use the contents of the syringe for culture, inoculating animals and preparing stained smears.

Skin biopsy: Skin should be carefully shaved and disinfected before snip biopsies are made.

\section{Wild rodents}

Wild rodents may have Leishmania organisms in either damaged or normallooking skin; there may or may not be visceral involvement.

- Examine the skin carefully, as skin lesions are often not obvious.

- The skin is usually heavily contaminated with fungi and bacteria. If the animal is dead, scrub the skin with soap and water and rinse thoroughly under running water before shaving and swabbing with alcohol. For live animals (usually anaesthetized), thoroughly cleanse lesions and apparently normal skin with alcohol, preferably on several occasions over 2-3 days before the biopsy is to be performed.

- For ulcerated lesions, take a biopsy sample from dry skin with a punch or scalpel; use scissors and forceps for ear lesions and apparently normal skin.

- Transfer the tissue to a Petri dish containing sterile saline or prolinebalanced salt solution with a high concentration of penicillin (100 000 $\mathrm{IU} / \mathrm{ml})$.

- Dissect out any remaining fur with sterile instruments.

- Wash the remaining tissue by passage through several changes of saline or proline-balanced salt solution containing penicillin (100 $000 \mathrm{IU} / \mathrm{ml})$.

- Cut the tissue into very thin slices or grind it in a tissue grinder.

- Inoculate culture media and animals, and prepare stained smears.

\section{Isolation from wild vectors}

The method used for isolation from insects depends on whether they can be transported live to a laboratory. Direct inoculation of sandfly guts into culture medium is associated with a high probability of fungal contamination of any resulting culture, and the process is particularly risky in the field. 


\section{Laboratory isolation}

- Rinse the sandflies thoroughly in sterile saline or proline-balanced salt solution.

- Place each fly in a drop of sterile saline on a sterile microscope slide, dissect out the gut, and examine under a microscope.

- When a gut is found to contain promastigotes, either express the contents into the salt solution used for dissection and inject directly into culture medium, or drop the entire gut into the medium.

A surer method is to inoculate an experimental animal, such as a hamster, with the gut contents of the infected sandfiy and reisolate Leishmania from the hamster.

\section{Field isolation}

In-vitro isolation in the field is usually extremely difficult because of the possibility of bacterial and fungal contamination of cultures. Successful isolations into culture can be made, but they require a great deal of expertise. In-vivo isolations are likely to be far more successful under field conditions. lnoculate any positive guts directly into the snouts, feet and peritoneal cavities of hamsters, then proceed as described below.

\section{In-vivo isolation}

In-vivo isolation involves inoculation of material containing Leishmania organisms into susceptible laboratory animals, the most suitable animal being the golden hamster. Other animals, such as various strains of inbred (particularly BALB/c) and outbred mice are used on occasion, but they are not as uniformly susceptible to Leishmania species as the hamster.

Hamsters are inoculated either intradermally into the nose and dorsal surfaces of the rear feet for dermatotropic Leishmania or intraperitoneally for visceral organisms. Leishmania are then isolated into culture from the hamster in one of the following ways:

- Wait until a lesion becomes apparent (cutaneous leishmaniasis) at the site of inoculation, then culture from it.

- Note the exact site of inoculation of the hamster; wait 7-14 days, then kill the animal. Excise the tissue into which the inoculation was made, and culture from it in the usual way. This method has the distinct advantage that it avoids the wait for a lesion to become apparent; this delay may be as long as 1 year with some strains of L. braziliensis.

- Kill the animal, and make stained smears from the liver and spleen. If positive, remove portions of positive tissue aseptically and inoculate into culture medium. 


\section{Laboratory maintenance of organisms}

The correct choice of culture medium is especially important in attempts to establish Leishmania organisms in culture. Unfortunately, it is diflicult to predict which species will grow easily in which culture medium. If in doubt, choose one or more of the blood- agar-based media described below; these are widely used for both Old and New World Leishmania.

\section{Biphasic blood-based media}

NNN medium: The agar is made by heating together in a flask $1.4 \mathrm{~g}$ of plain, nonnutrient agar, $0.6 \mathrm{~g}$ of $\mathrm{NaCl}$ and $90 \mathrm{ml}$ of distilled water. Heat the contents of the flask until the agar melts; keep the contents well mixed, or the agar will burn on the bottom of the flask. Transfer the appropriate amount of molten agar directly into the culture vessels. Sterilize the agar by autoclaving the culture tubes at $121^{\circ} \mathrm{C}$ for $15 \mathrm{~min}$. Allow the agar to cool to about $50^{\circ} \mathrm{C}$ and add aseptically collected, defibrinated rabbit blood to a final concentration of approximately $15 \%$. Mix the blood-agar by rolling the tubes in an upright position between the hands. Place the tubes in a sloped position until the agar has set, then stand them upright and transfer to a refrigerator or place in iced water. The liquid phase consists of the water that condenses at the bottom of the slopes; no additional liquid phase is added. The rapid cooling of freshly made slopes by transfer to a refrigerator or iced water greatly increases the amount of water of condensation that accumulates.

USAMRU medium (Difco blood-agar medium): This is a much richer medium than NNN, which is especially useful in the isolation of nutritionally more fastidious organisms, such as L. braziliensis. For the solid phase, $4 \mathrm{~g}$ of 'Bacto' blood-agar base (Difco) are added to $100 \mathrm{ml}$ of water. The method is the same as for NNN medium. The liquid phase is also as for NNN medium; if additional liquid is required, a few drops of sterile distilled water may be added.

Evans modified Tobie medium: This rich biphasic medium has been used for isolation of a wide variety of Old and New World Leishmania species. The solid phase consists of $0.3 \mathrm{~g}$ of beef extract, $0.5 \mathrm{~g}$ of bacteriological peptone, $0.8 \mathrm{~g}$ of NaCl, $2.0 \mathrm{~g}$ of agar amd $100 \mathrm{ml}$ of distilled water. Mix and heat the ingredients in a flask as for NNN medium. Transfer the molten agar into culture tubes and autoclave at $121^{\circ} \mathrm{C}$ for $15 \mathrm{~min}$. Cool the sterilized agar to about $55{ }^{\circ} \mathrm{C}$, then add defibrinated horse blood (inactivated by heating at $56{ }^{\circ} \mathrm{C}$ for $30 \mathrm{~min}$ ) to give a final concentration of approximately $15 \%$. Mix and slope as for NNN medium. The liquid phase is proline- containing balanced salt solution. Add $0.2-0.3 \mathrm{ml}$ of the liquid phase to the agar slope immediately before inoculation. 
Note on use of blood other than rabbit blood in biphasic medium: Quite often, rabbit blood is not readily available for inclusion in biphasic media such as NNN or USAMRU. In such cases, mammalian blood other than rabbit may be used. Sheep, horse and human blood have all been used, but it is worth experimenting with whatever blood is readily available. With blood other than rabbit, use it either defibrinated or with an anticoagulant, but always heat-inactivate it $\left(56^{\circ} \mathrm{C} ; 30 \mathrm{~min}\right)$, and increase the concentration of agar in the medium to $2 \%$.

Sterility checking of blood-agar: Incubate freshly made blood-agar medium at $37{ }^{\circ} \mathrm{C}$ for $24 \mathrm{~h}$, and examine the surface of the blood-agar for signs of bacterial growth. Discard immediately any medium that shows such signs.

Storage: Store at $4{ }^{\circ} \mathrm{C}$. If a separate liquid phase is needed, add this just before the medium is used. These media are best used within 1 week. Discard after 3 weeks at $4{ }^{\circ} \mathrm{C}$.

Schneider Drosophila medium: This is a commercially available, liquid, insect-tissue culture medium, which, supplemented with 100, 200 or even 300 $\mathrm{ml} / \mathrm{l}$ fetal calf serum, has been widely used for the isolation and bulk cultivation of Leishmania spp. It is very expensive and with somewhat variable performance. To $100 \mathrm{ml}$ of Schneider Drosophila medium (revised) with Lglutamine, add 10,20 or $30 \mathrm{ml}$ of heat-inactivated $\left(56^{\circ} \mathrm{C}\right.$ for $\left.30 \mathrm{~min}\right)$ fetal calf serum.

Media for maintenance and bulk cultivation: For large-volume cultures, liquid media are more convenient than biphasic ones. MEM:FCS:EBLB medium is a nutritionally rich liquid medium suitable for the growth (but not the isolation) of almost any Leishmania species. It consists of $100 \mathrm{ml}$ of minimum essential medium with Earle salts (modified, autoclavable), $3 \mathrm{ml}$ of sodium bicarbonate solution ( $75 \mathrm{~g} / \mathrm{l}), 5 \mathrm{ml}$ of Evans blood lysate broth and $10 \mathrm{ml}$ of heat-inactivated fetal calf serum.

Evans blood lysate broth is prepared from $1.5 \mathrm{~g}$ of tryptose, $1.0 \mathrm{~g}$ of casein hydrolysate, $1.0 \mathrm{~g}$ of liver digest, $0.15 \mathrm{~g}$ of L-proline, $0.68 \mathrm{~g}$ of $\mathrm{KH}_{2} \mathrm{PO}_{4}$, $0.17 \mathrm{~g}$ of $\mathrm{NaOH}$ and $100 \mathrm{ml}$ of distilled water. To obtain a final $\mathrm{pH}$ of 7.37.4 , adjust if necessary with either $1 \mathrm{~mol} / 1 \mathrm{HCl}$ or $1 \mathrm{~mol} / \mathrm{l} \mathrm{NaOH}$. Dissolve the solid ingredients in the distilled water and sterilize by autoclaving at $121^{\circ} \mathrm{C}$ for $15 \mathrm{~min}$. After cooling, add $15 \mathrm{ml}$ of an aseptically prepared blood lysate prepared from aseptically collected whole blood (human, equine, ovine, caprine and rabbit blood appear to be equally good) either taken into an anticoagulant or delibrinated. Centrifuge at approximately $3000 \times g$ for 10 min and remove the liquid portion (serum or plasma). Wash the packed blood cells twice by resuspension in an equal volume of either sterile isotonic saline or proline balanced salt solution and recentrifuge $(3000 \times g$ for $10 \mathrm{~min})$. Lyse 
the washed blood cells by adding an equal volume of sterile distilled water. Mix the water and blood cells thoroughly and use this solution as the blood lysate to complete the medium. The medium will be cloudy at this point as a result of the cellular debris added in the blood lysate; clarify it by aseptic centrifugation at $15000 \times \mathrm{g}$ for at least $30 \mathrm{~min}$. Decant all the clear supernatant, taking care not to disturb the pellet. Bottle and store the supernatant at $4{ }^{\circ} \mathrm{C}$.

Semi-solid media are valuable as transport media and for reviving ailing cultures.

'Sloppy Evans' consists of $80 \mathrm{ml}$ of proline balanced salt solution, $0.1 \mathrm{~g}$ of bacteriological peptone, $0.03 \mathrm{~g}$ of beef extract, $10 \mathrm{ml}$ of washed packed horse blood cells, $10 \mathrm{ml}$ of heat-inactivated fetal calf serum and $0.3 \mathrm{~g}$ of agar (plain nonnutrient). Mix all the ingredients except the packed cells and fetal calf serum in a flask or screw- capped bottle. Sterilize by autoclaving $\left(121{ }^{\circ} \mathrm{C}\right.$ for $15 \mathrm{~min}$ ), cool to about $50^{\circ} \mathrm{C}$, and add the packed cells and fetal calf serum; mix well and dispense while still molten into suitable sterile culture tubes.

Semi-solid Locke blood-agar medium: Locke solution is made up of $9.2 \mathrm{~g}$ of $\mathrm{NaCl}, 0.24 \mathrm{~g}$ of $\mathrm{CaCl}_{2}, 0.15 \mathrm{~g}$ of $\mathrm{NaHCO}_{3}, 0.42 \mathrm{~g}$ of KCl, $1.0 \mathrm{~g}$ of D-glucose and $1000 \mathrm{ml}$ of distilled water. The blood-agar base consists of $2.5 \mathrm{~g}$ of agar (plain nonnutrient), $1.0 \mathrm{~g}$ of bacteriological peptone, $0.5 \mathrm{~g}$ of $\mathrm{NaCl}$ and 100 $\mathrm{ml}$ of distilled water. Mix seven parts of Locke solution with one part of molten blood-agar base, adjust the $\mathrm{pH}$ to 7.4 and autoclave at $121^{\circ} \mathrm{C}$ for 15 min. Allow to cool to about $50{ }^{\circ} \mathrm{C}$, and add defibrinated rabbit blood to a concentration of approximately $10 \%$. Mix thoroughly, then dispense into sterile culture vessels.

\section{Cryopreservation of Leishmania}

It is important to preserve organisms as soon as conveniently possible after initial isolation. The frozen sample is known as a stabilate. Cryopreserved Leishmania can be stored in low-temperature mechanical freezers $\left(-70^{\circ} \mathrm{C}\right)$, in solid carbon dioxide (containers $\left(-76^{\circ} \mathrm{C}\right)$ or in liquid nitrogen containers $\left(-196^{\circ} \mathrm{C}\right)$. Each method has its drawbacks; mechanical deep-freezers are liable to break down or the electricity supply may not be reliable; liquid nitrogen and carbon dioxide storage both rely on the ready availability of regular supplies.

Take great care to avoid cryogenic burns and accidents from exploding containers when freezing, storing or thawing samples. Always wear protective gloves and a total face mask or safety spectacles. Handle frozen tubes and ampoules with blunt-ended forceps. 


\section{Record-keeping}

Record-keeping is vitally important, and a cryopreservation record book or chart must be kept. It should contain details of the samples, their location in the cryobank and a brief history of each stabilate with its international code number. All tubes, ampoules and capillary tubes in the bank should be clearly labelled; any material that has lost its label must be discarded.

Keep a careful record of what is removed from the cryobank. Do not allow general access, but give one person the overall responsibility for all issues from and receipts into the bank, with concomitant record-keeping. This person and one or possibly two assistants should be the only people with direct access to the bank; all must know the method of record-keeping.

\section{Freezing}

Freezing is a simple process for Leishmania and does not require sophisticated apparatus. It is carried out slowly in the presence of a cryoprotectant.

Using aseptic technique, transfer a measured volume of culture into a sterile glass tube or other container kept on ice. Actively dividing promastigotes in mid-logarithmic growth phase seem to survive freezing and thawing better than nondividing cells. Ideally, cultures to be frozen should have a minimum promastigote concentration of 1 million per ml. Stabilates with lower concentrations take longer to re-establish in culture. Add sterile cryoprotectant (glycerol sterilized by autoclaving) to give a concentration of $7.5-10 \%$ in the final volume or filtered sterilized dimethyl sulfoxide to a final concentration of 5.0-7.5\%. Mix thoroughly. Transfer the cryoprotected samples to the sterile containers in which they are to be frozen. These may be 2-ml (38.0 $\times 12.5 \mathrm{~mm}$ ) plastic freezing tubes with airtight screw caps, hard glass, heatsealed ampoules or capillary tubes made of glass or plastic. Do not overfill: the containers should be no more than two-thirds full. Label them, and seal them. Make sure that those made of glass are correctly heat-sealed; failure to do so can result in violent explosion when the containers are thawed after storage in liquid nitrogen. It is safer to store glass containers in the vapour phase above the surface of liquid nitrogen, but special liquid nitrogen refrigerators are required for this.

Slowly freeze the containers with the stabilates at a rate of about $1{ }^{\circ} \mathrm{C}$ per minute. This can be done in several ways:

- Place the containers in an insulated vessel, such as a glass or metal tube surrounded by a jacket of expanded polystyrene or similar insulating material, about $3 \mathrm{~cm}$ thick. Place in a freezer at $-70^{\circ} \mathrm{C}$ overnight.

- Cool the samples to $4{ }^{\circ} \mathrm{C}$ and keep them at this temperature for a minimum of $1 \mathrm{~h}$; they can be left overnight if necessary. Transfer to a freezer at 
$-20{ }^{\circ} \mathrm{C}$ and leave for $24 \mathrm{~h}$; then, remove to a freezer at $-70{ }^{\circ} \mathrm{C}$ for at least 24 $\mathrm{h}$. The containers can be stored permanently at this temperature or transferred into liquid nitrogen $\left(-196^{\circ} \mathrm{C}\right)$ or solid carbon dioxide $\left(-76^{\circ} \mathrm{C}\right)$.

- Place the containers in a special vessel which fits into the mouth of a liquid nitrogen Dewar flask; slow freezing will take place in the liquid nitrogen vapour over a period of $24 \mathrm{~h}$.

- Programmable freezing units may be used if available. In this case, the rate of freezing should be $1{ }^{\circ} \mathrm{C} / \mathrm{min}$ from $25^{\circ} \mathrm{C}$ to $2{ }^{\circ} \mathrm{C}$, then $5{ }^{\circ} \mathrm{C} / \mathrm{min}$ from $2{ }^{\circ} \mathrm{C}$ to $-18{ }^{\circ} \mathrm{C}$ and $10{ }^{\circ} \mathrm{C} / \mathrm{min}$ from $-18{ }^{\circ} \mathrm{C}$ to $-70{ }^{\circ} \mathrm{C}$ and below.

Quickly transfer the containers with their stabilates to their final storage place, making sure that they do not become warm during transfer.

Before discarding the cultures from which the stabilates were made, thaw the contents of one container of each frozen isolate, and culture from it to check the viability of the stabilate. If no growth is obtained, make a fresh stabilate from the original culture. 



\section{Annex 3 \\ WHO recommended case definitions}

\section{Visceral leishmaniasis}

\section{Clinical description}

An illness with prolonged irregular fever, splenomegaly and weight loss as its main symptoms. In endemic malarious areas, visceral leishmaniasis should be suspected when fever lasts for more than 2 weeks and no response has been achieved with antimalarial medicines (assuming that drug-resistant malaria has also been considered).

\section{Laboratory criteria for diagnosis}

- positive parasitology (stained smears from bone marrow, spleen, liver, lymph node, blood or culture of the organism from a biopsy or aspirated material); and

- positive serology (IFAT, ELISA, rK39, direct agglutination test).

- positive PCR and related techniques

\section{Case classification by WHO operational definition}

A case of visceral leishmaniasis is a person showing clinical signs (mainly prolonged irregular fever, splenomegaly and weight loss) with serological and/or parasitological confirmation. ${ }^{11}$

\section{Post-kala-azar dermal leishmaniasis (PKDL)}

Special efforts should be made to trace PKDL in the community, because patients with PKDL have only skin manifestations and usually do not attend clinics or see skin specialists only. PKDL can be confused with pauci or multibacillary leprosy. The skin lesions may also mimic other skin conditions.

Case classification ${ }^{12}$

Probable PKDL: A patient from an area endemic for kala-azar with multiple hypopigmented macules, papules or plaques or nodules with no sensitivity

\footnotetext{
11 WHO. Recommended surveillance standards, 2nd ed., Geneva, World Health Organization, 1999.

12 WHO/SEARO. Indicator toolkit for the visceral leishmaniasis elimination initiative. New Delhi, 2010.
} 
loss Confirmed PKDL: A patient from an area endemic for kala-azar with multiple hypopigmented macules, papules, plaques or nodules who is parasite- or PCR-positive in a slit skin smear or biopsy.

\section{Cutaneous leishmaniasis}

\section{Clinical description}

Appearance of one or more lesions, typically on uncovered parts of the body. The face, neck, arms and legs are the commonest sites. At the site of inoculation, a nodule appears, which may enlarge to become an indolent ulcer. The sore remains in this stage for a variable time before healing and typically leaves a depressed scar. Other atypical forms may occur. In some individuals, certain strains can disseminate and cause mucosal lesions. These sequelae involve nasopharyngeal tissues and can be disfiguring.

\section{Laboratory criteria for diagnosis}

- positive parasitology (stained smear or culture from the lesion)

- mucocutaneous leishmaniasis only: positive serology (IFAT, ELISA)

\section{Case classification by WHO operational definition ${ }^{13}$}

A case of cutaneous leishmaniasis is a person showing clinical signs (skin or mucosal lesions) with parasitological confirmation of the diagnosis (positive smear or culture) and/or, for mucocutaneous leishmaniasis only, serological diagnosis.

13 WHO. Recommended surveillance standards, 2nd ed, Geneva, 1999, WHO/CDS/CSR/ISR/ 99.2. 


\section{Annex 4 \\ Procedures for splenic aspiration and grading of parasites}

Splenic aspiration should be performed only if the following conditions are met:

- absence of clinical contraindication(s):

- signs of active bleeding (e.g. epistaxis, rectal bleeding, skin bruises)

- jaundice (a potential marker of liver dysfunction)

- pregnancy

- spleen barely palpable

- bad general condition (e.g. cardiovascular shock, altered consciousness)

- absence of biological contraindication(s):

- severe anaemia (haemoglobin count $\leq 5 \mathrm{~g} / \mathrm{l}$ )

- difference in prothrombin time between patient and control $>5 \mathrm{~s}$

- platelet count $<40000 / \mathrm{ml}$

- rapid access to blood transfusion in case of bleeding

The two important prerequisites for the safety of the procedure are rapidity, so that the needle remains within the spleen for less than $1 \mathrm{~s}$; and precision, so that the entry and exit axes of the aspirating needle are identical to avoid tearing the splenic capsule.

The procedure is as follows:

1. Clean three glass slides and label them with patient's name, date and the words 'splenic aspirate'. Have culture medium ready (if available) and labelled in the same way as the slides. Attach a $1^{1 / 4}$-inch $\times 21$ gauge $(32 \times 0.8-\mathrm{mm})$ needle to a $5-\mathrm{ml}$ syringe. Place all items on a table at the bedside.

2. Inform the patient about the procedure. Check all clinical and biological contraindications again. Palpate the spleen and outline its margins on the patient's abdomen with a pen. For safety, the spleen should be palpable at least $3 \mathrm{~cm}$ below the costal margin on expiration. Use an 
alcohol swab to clean the skin at the site of aspiration, and allow the skin to dry.

3. With the 21 -gauge $(0.8-\mathrm{mm})$ needle attached to the 5 - $\mathrm{ml}$ syringe, just penetrate the skin, midway between the edges of the spleen, $2-4 \mathrm{~cm}$ below the costal margin. Aim the needle cranially at an angle of $45^{\circ}$ to the abdominal wall. The actual aspiration is done as follows: pull the syringe plunger back to approximately the 1- $\mathrm{ml}$ mark to apply suction, and with a quick in-and-out movement push the needle into the spleen to the full needle depth and then withdraw it completely, maintaining suction throughout.

4. For young, restless children, have two assistants hold the child (arms folded across the chest, with shirt raised to obstruct the line of vision, and pelvis held firmly). Carry out the aspiration as a single-stage procedure, using the same landmarks, angles and suction as in step 3, all in one quick motion. The insertion should be timed with the patient's breathing so that the diaphragm is not moving; this should be done during fixed expiration if the child is crying. Only a minute amount of splenic material is obtained, but this is sufficient for culture and smear.

5. If culture is available: slowly pull the plunger back to the 2-3-ml mark, and, using sterile techniques, insert the needle into a tube containing culture medium and briskly push the plunger into the barrel to expel the contents of the needle onto the side walls of the tube. If necessary, repeat once or twice until splenic material is visible in the tube. Replace the cap on the tube and invert to wash splenic material on the side of the tube. Repeat the procedure for the second tube of culture medium. Sterile techniques are essential throughout.

6. Expel material (or additional material if culture is available) gently onto glass slides, holding the needle tip on the surface of the slide. Immediately spread evenly with the needle, using a linear (not circular) motion. The smear should be slightly thinner than a thick blood film for malaria. Remove the needle, and use the end of it to obtain additional material from the tip of the syringe and spread it on slides. Further material found on the end of the plunger may be dabbed directly onto a slide and spread. Allow the slides to dry.

7. Write the time of aspiration on the patient's chart, with the instructions: "Record pulse and blood pressure every half hour for $4 \mathrm{~h}$, then every hour for $6 \mathrm{~h}$. Patient must remain in bed for $12 \mathrm{~h}$." Ensure that the patient understands the instructions. Enter the procedure in the notes, and sign. 
8. Take the slides (and medium) to the laboratory. Slides are stained with Giemsa as for a thin malaria film and examined under oil immersion. The average amastigote density is graded as follows: ${ }^{14}$

$6+:>100$ parasites per field (viewed with a $10 \times$ eyepiece and $100 \times$ oil- immersion lens)

5+: $10-100$ parasites per field

4+: $1-10$ parasites per field

3+: $1-10$ parasites per 10 fields

2+: $1-10$ parasites per 100 fields

1+: $1-10$ parasites per 1000 fields

0: 0 parasite per 1000 fields

Parasite grading has several uses. It increases the sensitivity of parasite detection, provides an objective measure of the speed of response to treatment, distinguishes quickly between slow responders and nonresponders, and provides an indication of parasite load that is useful in research.

\footnotetext{
${ }^{14}$ Adapted from Chulay JD, Bryceson AD. Quantitation of amastigotes of Leishmania donovani in smears of splenic aspirates from patients with visceral leishmaniasis. American Journal of Tropical Medicine and Hygiene, 1983; 32:475-9.
} 



\section{Annex 5 \\ Performance of the rK39 rapid diagnostic test ${ }^{15}$}

The utility of a rapid diagnostic test for visceral leishmaniasis lies in its simplicity. Several brands of test with rK39 antigen are available. Operators should always read the package insert carefully, and follow the manufacturer's instructions. This is especially important with regard to the type of specimen used: serum or whole blood. Some brands can be used only with serum, while others can be used with whole blood collected by finger prick.

\section{Test procedure}

Refer always to the specifications given by the manufacturer.

In general, the test procedure is as follows (Figure A5.1):

1. Remove the test strip from the pouch and place it on a flat surface

2. Place a specified amount of patient specimen (serum or finger-prick blood) on the absorbent pad on the bottom of the strip

3. Add the specified amount of buffer provided

4. Read the result after 10-20 min, according to the manufacturer's instructions

Figure A5.1.

Procedure for performing the rK39 rapid diagnostic test

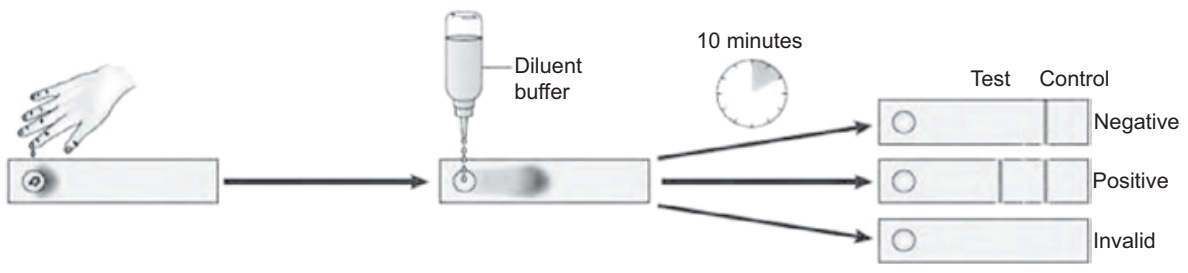

15 UNiCEF/UNDP/World Bank/WHO Special Programme for Research and Training in Tropical Diseases. The use of visceral leishmaniasis rapid diagnostic tests. 2nd ed. Geneva, World Health Organization, 2008. 
Some brands require a slightly different procedure, for example:

1. Take a test tube or a U-bottom microtitre plate

2. Add a specified amount of buffer to the tube or well

3. Add a specified amount of specimen (blood or serum) to the tube or well and mix

4. Immerse the test strip into the buffer-specimen mixture

5. Read the result after 10-20 min, according to the manufacturer's instructions

\section{Points to consider for optimizing use of rapid diagnostic tests:}

- Have a clear management plan to deal with positive and negative results

- Follow biosafety standards and precautions for handling blood and other body fluids

- Ensure proper storage conditions

- Do not use damaged or expired tests

- Adhere strictly to the manufacturer's instructions

- Use test kits within $1 \mathrm{~h}$ of removal from pouch

- Read the results within the time specified by the manufacturer

- Do not reuse a test

\section{Interpretation of the test}

Positive result: When both control and test lines appear, the sample tested has antibodies against recombinant K39 antigen of Leishmania. Even a faint line should be considered positive.

Negative result: When only the control line appears, there are no antibodies against recombinant K39 antigen of Leishmania present in the patient's sample.

Invalid result: When no control line appears, a fresh patient sample should be tested with a new strip.

\section{Advantages and disadvantages of the rK39 test}

\section{Advantages}

- Simple to perform with minimal training.

- Does not require a laboratory.

- Can be performed with finger-prick whole blood, serum or plasma.

- Kits can be transported and stored at ambient temperature (up to $30^{\circ} \mathrm{C}$ ).

- Results are available within 10-20 min. 


\section{Disadvantages}

- Cannot distinguish between active cases and relapse in previously treated cases. Therefore, interpretation must always be accompanied by clinical case definition.

- In patients with advanced HIV infection, a negative result does not rule out a diagnosis of visceral leishmaniasis. 



\section{Annex 6 \\ Costs of medicines in current use for the treatment of leishmaniasis}

Table A6.1.

Drug prices (January 2010)

\begin{tabular}{|c|c|c|}
\hline Compound & $\begin{array}{l}\text { Commercial name and } \\
\text { manufacturer }\end{array}$ & Price information ${ }^{\mathrm{a}}$ \\
\hline mphotericin B deoxycholate & $\begin{array}{l}\text { Different names in different } \\
\text { countries }\end{array}$ & $\begin{array}{l}\text { Variable, but median is US } \\
\$ 7.5 \text { per } 50-\mathrm{mg} \mathrm{vial}^{\mathrm{b}}\end{array}$ \\
\hline Liposomal amphotericin B & $\begin{array}{l}\text { AmBisome®, Gilead, USA } \\
\text { Single source }\end{array}$ & $\begin{array}{l}\text { WHO negotiated price, US } \\
\$ 18 \text { per } 50-\text { mg vial }^{c}\end{array}$ \\
\hline Miltefosine & $\begin{array}{l}\text { Impavido®, Paladin, Canada } \\
\text { Single source }\end{array}$ & $\begin{array}{l}\text { WHO-negotiated prices: }{ }^{\text {d }} \\
\text { For adults: } € 45.28-54.92 \\
\text { for } 56(50-\mathrm{mg}) \text { capsules } \\
\text { For children: } € 34.36-39.3 \\
\text { for } 56(10-\mathrm{mg}) \text { capsules }\end{array}$ \\
\hline Paromomycin & $\begin{array}{l}\text { Paromomycin, Gland } \\
\text { Pharma, India } \\
\text { Single source }\end{array}$ & $\begin{array}{l}\text { Approximate price, US\$ } 15 \\
\text { per adult course of } 21 \text { days }\end{array}$ \\
\hline $\begin{array}{l}\text { Sodium stibogluconate } \\
\text { (SSG) }\end{array}$ & Pentostam $®$, GSK & $\begin{array}{l}66.43 \mathrm{GBP} \text { per vial } 100 \mathrm{ml} \text {, } \\
100 \mathrm{mg} / \mathrm{ml}^{\mathrm{e}}\end{array}$ \\
\hline $\begin{array}{l}\text { Generic sodium } \\
\text { stibogluconate }\end{array}$ & $\begin{array}{l}\text { SSG, Albert David, India } \\
\text { Single source }\end{array}$ & $\begin{array}{l}€ 5.65 \mathrm{per} 30-\mathrm{ml} \text { vial of } \\
100 \mathrm{mg} / \mathrm{ml}^{\mathrm{e}}\end{array}$ \\
\hline Meglumine antimoniate & $\begin{array}{l}\text { Glucantime } ® \text {, Aventis } \\
\text { Single source }\end{array}$ & $\begin{array}{l}\text { WHO-negotiated price: } \\
\text { US } \$ 1.2 \text { per } 5-\mathrm{ml} \text { vial of } \\
81 \mathrm{mg} / \mathrm{ml}\end{array}$ \\
\hline \multicolumn{3}{|c|}{$\begin{array}{l}\text { a Prices as quoted by manufacturers in the currency as originally quoted } \\
\text { b UNICEF. Sources and prices of selected medicines for children, } 2 \text { nd ed., } 2010 \text {. } \\
\text { ' Price valid until December } 2010 \text {. The price will be re-established every year, with a ceiling of US\$ } \\
20 \text { per vial } \\
\text { d Prices depend on size of order } \\
\text { e Price as quoted in British National Formulary } 59 \\
\text { † Valid for governments, United Nations organizations and nongovernmental organizations } \\
\text { Information on access to drugs at the WHO-negotiated price is available at www.who.int. }\end{array}$} \\
\hline
\end{tabular}

Note: costs are given by the companies in the indicated currencies that are maintained to avoid any potential variations 
Table A6.2.

Price per visceral leishmaniasis treatment (January 2010)

\begin{tabular}{|c|c|c|}
\hline Compound & Treatment regimen & Drug cost in US\$a \\
\hline L-Amb 10 mg/kg & 1 day & 126 \\
\hline L-Amb 20 mg/kg & 2-4 days & 252 \\
\hline $\begin{array}{l}\text { Amphotericin B deoxycholate } 1 \mathrm{mg} / \mathrm{kg} \\
\text { (alternating days) }\end{array}$ & 30 days & 20 \\
\hline MF 100 mg/day & 28 days & $65-150$ \\
\hline PM 15 mg/kg/day & 21 days & 15 \\
\hline SSG 20 mg/kg/day & 30 days & 55,8 \\
\hline MA 20 mg/kg/day & 30 days & 59,3 \\
\hline L-Amb 5 mg/kg + MF 100 mg/day & 8 days & $88,2-109,5$ \\
\hline L-Amb 5 mg/kg + PM 15 mg/kg/day & 11 days & 79 \\
\hline MF 100 mg/day + PM 15 mg/kg/day & 10 days & $30,2-60,7$ \\
\hline (SSG $20 \mathrm{mg}+\mathrm{PM} 15 \mathrm{mg}$ )/kg/day & 17 days & 44 \\
\hline
\end{tabular}

a For a patient weighing $35 \mathrm{~kg}$. Calculations for SSG and MF based on exchange rate of $€ 1=$ US\$ 1.41 (28 January 2010). Price range of miltefosine depends on order volume. Price is based on generic SSG.

$\mathrm{L}-\mathrm{Amb}=$ liposomal amphotericin $\mathrm{B}, \mathrm{MF}=$ =miltefosine, $\mathrm{PM}=$ paromomycin, $\mathrm{SSG}=$ sodium stibogluconate, $M A=$ meglumine antimoniate

Table A6.3.

Price per cutaneous leishmaniasis treatment (January 2010)

\begin{tabular}{|c|c|c|}
\hline Compound & Treatment regimen & Drug cost in US\$a \\
\hline SSG systemic, 20 mg/kg/day & 20 days & 37,2 \\
\hline SSG intralesional ${ }^{b}$ & Until lesion is healed & 12 \\
\hline MA systemic, 20 mg/kg per day & 20 days & 39,5 \\
\hline MA intralesional ${ }^{b}$ & Until lesion is healed & 13,2 \\
\hline Pentamidine & Up to 4 months & Free (donation programme) \\
\hline
\end{tabular}

a For a patient weighing $35 \mathrm{~kg}$. Calculations for sodium stibogluconate based on exchange rate of $€$ $1=$ US $\$ 1.41$ (28 January 2010) Price is based on generic SSG.

${ }^{\mathrm{b}}$ Intralesional treatment is commonly estimated at a third of the cost of systemic treatment SSG=sodium stibogluconate, $\mathrm{MA}=$ meglumine antimoniate 
This report makes recommendations on new therapeutic regimens for visceral and cutaneous leishmaniasis, on the use of rapid diagnostic tests, details on the management of Leishmania-HIV coinfection and consideration of social factors and climate change as risk factors for increased spread.

Recommendations for research include the furtherance of epidemiological knowledge of the disease and clinical studies to address the lack of an evidence-based therapeutic regimen for cutaneous and mucocutaneous leishmaniasis and post-kala-azar dermal leishmaniasis (PKDL).

This report not only provides clear guidance on implementation but should also raise awareness about the global burden of leishmaniasis and its neglect. It puts forward directions for formulation of national control programmes and elaborates the strategic approaches in the fight against the leishmaniases. The committee's work reflects the latest scientific and other relevant developments in the field of leishmaniasis that can be considered by Member States when setting national programmes and making public health decisions.

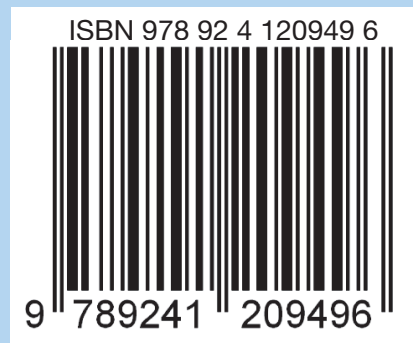

